\title{
ON TORUS ACTIONS OF HIGHER COMPLEXITY
}

\author{
JÜRGEN HAUSEN ${ }^{1}$, CHRISTOFF HISCHE $^{1}$ and MILENA WROBEL ${ }^{2}$ \\ ${ }^{1}$ Fachbereich Mathematik, Universität Tübingen, Auf der Morgenstelle 10, \\ 72076 Tübingen, Germany; \\ email: juergen.hausen@uni-tuebingen.de, hische@math.uni-tuebingen.de \\ ${ }^{2}$ Institut für Mathematik, Universität Oldenburg, 26111 Oldenburg, Germany; \\ email: milena.wrobel@uni-oldenburg.de
}

Received 17 May 2018; accepted 20 September 2019

\begin{abstract}
We systematically produce algebraic varieties with torus action by constructing them as suitably embedded subvarieties of toric varieties. The resulting varieties admit an explicit treatment in terms of toric geometry and graded ring theory. Our approach extends existing constructions of rational varieties with torus action of complexity one and delivers all Mori dream spaces with torus action. We exhibit the example class of 'general arrangement varieties' and obtain classification results in the case of complexity two and Picard number at most two, extending former work in complexity one.
\end{abstract}

2010 Mathematics Subject Classification: 14L30, 14M25, 14J45 (primary)

\section{Introduction}

This article contributes to the study of algebraic varieties with torus action. Here, a torus is an algebraic group $\mathbb{T}$ isomorphic to the $k$-fold direct product $\mathbb{T}^{k}$ of the multiplicative group $\mathbb{K}^{*}$ of the ground field $\mathbb{K}$, which is assumed to be algebraically closed and of characteristic zero. The presence of a torus action on a variety brings combinatorial aspects into the game, as becomes most evident in the case of toric varieties, that means normal varieties $Z$ containing a torus $\mathbb{T}_{Z}$ as an open subset such that the group structure of $\mathbb{T}_{Z}$ extends to an action on $Z$. The fundamental correspondence between toric varieties and fans observed in 1970 by Demazure [28] is the starting point of toric geometry, a meanwhile highly developed theory and continuously active field of research.

(c) The Author(s) 2019. This is an Open Access article, distributed under the terms of the Creative Commons Attribution licence (http://creativecommons.org/licenses/by/4.0/), which permits unrestricted re-use, distribution, and reproduction in any medium, provided the original work is properly cited. 
Beyond toric geometry, there are the $\mathbb{T}$-varieties $X$ of higher complexity, where one requires $X$ to be normal, the $\mathbb{T}$-action to be effective, meaning that only the neutral element acts trivially, and the complexity is the difference $\operatorname{dim}(X)-\operatorname{dim}(\mathbb{T})$. Torus actions of complexity one are studied as well since the 1970s; we mention the combinatorial approaches [53, 67], the geometric work [32-34, 61-64] on $\mathbb{K}^{*}$-surfaces and the more algebraic point of view based on trinomial relations $[41,42,47,58]$. In arbitrary complexity, we have the general approach via polyhedral divisors [1, 2], unifying in particular aspects of [34, 67]. Torus actions serve also as a model case for actions of more general algebraic groups, for example reductive ones, and the approaches just discussed reflect in this much more general theory; we restrict ourselves to refer to the seminal work [56] in complexity zero and [68] as a landmark in complexity one.

In the present article we consider $\mathbb{T}$-varieties of arbitrary complexity. The aim is to provide an explicit approach with close links to toric geometry, supporting, for instance, concrete, example-oriented work. Besides basic algebraic geometry and graded ring theory, only rudiments of toric geometry and Cox ring theory are needed; we refer to Section 2 for a brief summary.

Let us get into the matter by means of an example. Consider the fivedimensional smooth projective quadric $X \subseteq \mathbb{P}_{6}$. The automorphism group of $X$ is the orthogonal group $\mathrm{O}(7)$. Fixing a maximal torus $\mathbb{T} \subseteq \mathrm{O}(7)$, we turn $X$ into a $\mathbb{T}$-variety. Choosing suitable coordinates, we achieve that the quadric is given by

$$
X=V\left(T_{0}^{2}+T_{1} T_{2}+T_{3} T_{4}+T_{5} T_{6}\right) \subseteq \mathbb{P}_{6}
$$

and that the elements $t=\left(t_{1}, t_{2}, t_{3}\right)$ of the (three-dimensional) torus $\mathbb{T}=\mathbb{T}^{3}$ act on the points $[z]=\left[z_{0}, \ldots, z_{6}\right]$ of $\mathbb{P}_{6}$ via

$$
t \cdot[z]=\left[z_{0}, t_{1} z_{1}, t_{1}^{-1} z_{2}, t_{2} z_{3}, t_{2}^{-1} z_{4}, t_{3} z_{5}, t_{3}^{-1} z_{6}\right] .
$$

In particular, $\mathbb{T}$ acts diagonally. In order to link the situation in an optimal manner to toric geometry, we do a further step. Consider the torus $\mathbb{T}^{6} \subseteq \mathbb{P}_{6}$ consisting of all points with only nonzero homogeneous coordinates and the splitting

$$
\mathbb{T}^{6} \rightarrow \mathbb{T}^{3} \times \mathbb{T}^{3}, \quad t \mapsto\left(t_{1} t_{2}, t_{3} t_{4}, t_{5} t_{6}, t_{1}, t_{2}, t_{3}\right) .
$$

In terms of toric geometry, such a change of torus coordinates means passing to another describing fan of the projective space $\mathbb{P}_{6}$. More precisely, we switch over from the fan $\Delta_{6}$ with rays generated by the canonical basis vectors $e_{1}, \ldots, e_{6} \in \mathbb{Q}^{6}$ and $e_{0}=-e_{1}-\cdots-e_{6}$ to the fan $\Sigma$ with rays generated by

$$
e_{0}-e_{1}-e_{2}-e_{3}, e_{1}, e_{1}+e_{4}, e_{2}, e_{2}+e_{5}, e_{3}, e_{3}+e_{6} .
$$

In the new picture, our torus $\mathbb{T}$ is the second factor of the splitting $\mathbb{T}^{6}=\mathbb{T}^{3} \times \mathbb{T}^{3}$. The projection $\mathbb{T}^{6} \rightarrow \mathbb{T}^{3}$ onto the first factor mods out the $\mathbb{T}$-action and defines 
a $\mathbb{T}$-invariant rational map $\pi: \mathbb{P}_{6} \rightarrow \mathbb{P}_{3}$ which in terms of fans arises from the projection $\mathbb{Z}^{6} \rightarrow \mathbb{Z}^{3}$ onto the first factor mapping the rays of $\Sigma$ onto the rays of the fan $\Delta_{3}$ describing $\mathbb{P}_{3}$. Observe that the closure of the image $\pi(X)$ in $\mathbb{P}_{3}$ is

$$
Y=V\left(U_{0}+U_{1}+U_{2}+U_{3}\right) \subseteq \mathbb{P}_{3},
$$

the projective plane realized as a general hyperplane in the projective space. The restriction $\pi: X \rightarrow Y$ encodes important information on the $\mathbb{T}$-action and is what we will call a 'maximal orbit quotient'. Moreover, $X \subseteq \mathbb{P}_{6}$ is the zero set of a homogeneous quadratic polynomial $g$ and thus we have a homogeneous coordinate ring $\mathbb{K}\left[T_{0}, \ldots, T_{6}\right] /\langle g\rangle$. In case of the quadric $X$, the latter equals the Cox ring

$$
\mathcal{R}(X)=\bigoplus_{\mathrm{Cl}(X)} \Gamma(X, \mathcal{O}(D)),
$$

where the grading via the divisor class group $\mathrm{Cl}(X)$ is just the classical $\mathbb{Z}$ grading of its homogeneous coordinate ring. Also for $Y \subseteq \mathbb{P}_{3}$ we observe that the homogeneous coordinate ring equals the Cox ring.

Our approach replaces the ambient projective spaces of the above example with ambient toric varieties. Given a toric variety $Z$, say a complete one, Cox's quotient presentation delivers $Z$ as a quotient of an affine space. This allows us in particular, to associate with a closed subvariety $X \subseteq Z$ a generalized homogeneous coordinate ring. We call $X \subseteq Z$ an explicit variety if, roughly speaking, its generalized homogeneous coordinate ring equals its Cox ring. In Construction 3.5, we produce systematically explicit $\mathbb{T}$-varieties, where the idea is to reverse the process of the introductory example: one starts with an explicit variety $Y \subseteq Z_{Y}$ and then builds up via an elementary game an explicit variety $X \subseteq Z_{X}$ such that a direct factor $\mathbb{T}$ of the torus $\mathbb{T}_{Z} \subseteq Z$ leaves $X$ invariant and turns $X \subseteq Z$ into a $\mathbb{T}$-variety with maximal orbit quotient $X \rightarrow Y$. So, in the above example, the projective plane $Y \subseteq \mathbb{P}_{3}$ is the input and as output we obtain the quadric $X \subseteq \mathbb{P}_{6}$.

Explicit $\mathbb{T}$-varieties are designed to be directly accessible for concrete computation. Their geometry is strongly related to that of the ambient toric variety and, due to finite generation of their Cox ring, the combinatorial methods developed in [5, Section 3] apply; see Remark 2.10 for the precise interface and Section 5 for a collection of basic geometric properties. Let us say a few words about what kind of $\mathbb{T}$-varieties we obtain. First, Construction 3.5 generalizes the Cox ring based approach to rational $\mathbb{T}$-varieties of complexity one developed in [41, 47]; see Remark 5.14. For a general statement, recall the Mori dream spaces introduced by $\mathrm{Hu}$ and Keel [48]: these varieties behave perfectly with respect to the minimal model programme and are characterized as the $\mathbb{Q}$-factorial, 
projective varieties with finitely generated Cox ring. As a special case of the more general Theorem 3.10, we obtain the following.

THEOREM 1.1. Every Mori dream space with an effective torus action admits a presentation as an explicit $\mathbb{T}$-variety.

As a first major example class, we exhibit in Section 6 the general arrangement varieties. These are $\mathbb{T}$-varieties of complexity $c$ with maximal orbit quotient $\pi: X \rightarrow \mathbb{P}_{c}$ such that the critical values of $\pi$ form a general hyperplane arrangement in the projective space $\mathbb{P}_{c}$. We have already seen an example. The smooth projective quadric $X$ with its maximal torus action discussed at the beginning is a general arrangement variety of complexity two. The critical values of the maximal orbit quotient $\pi: X \rightarrow \mathbb{P}_{2}$ are the points of the lines

$$
V\left(T_{0}\right), \quad V\left(T_{1}\right), \quad V\left(T_{2}\right), \quad V\left(T_{0}+T_{1}+T_{2}\right) .
$$

Theorem 6.18 ensures that, for instance, all projective general arrangement varieties can be presented as explicit $\mathbb{T}$-varieties. In Section 7 we use the methods on explicit $\mathbb{T}$-varieties to investigate the geometry of general arrangement varieties. For example, Proposition 7.4 gives an explicit formula for the canonical divisor and Corollary 7.16 provides a purely combinatorial smoothness criterion.

Extending recent classification work in complexity one [31], we take a closer look at smooth general arrangement varieties of Picard number at most two. In Picard number one, we retrieve precisely the smooth projective quadrics; see Proposition 7.23. Similar to the case of complexity one, the situation in Picard number two is much more ample. For the case of complexity two, we obtain the following explicit descriptions; below, we say that a torus action on a variety is of true complexity $c$, if the action is of complexity $c$ and the variety does not admit a torus action of lower complexity. Note that being Mori dream spaces, the varieties listed below are indeed determined by their Cox ring together with an ample class; see Remark 5.7. Moreover, in Remark 8.11 we provide the defining data for the respective descriptions as explicit $\mathbb{T}$-varieties.

THEOREM 1.2. Every smooth projective general arrangement variety of true complexity two and Picard number two is isomorphic to precisely one of the following varieties $X$, specified by their Cox ring $\mathcal{R}(X)$, the matrix $\left[w_{1}, \ldots, w_{r}\right]$ of generator degrees and an ample class $u \in \mathrm{Cl}(X)=\mathbb{Z}^{2}$.

\begin{tabular}{|c|c|c|c|c|}
\hline No. & $\mathcal{R}(X)$ & {$\left[w_{1}, \ldots, w_{r}\right]$} & $u$ & $\operatorname{dim}(X)$ \\
\hline 1 & $\frac{\mathbb{K}\left[T_{1}, \ldots, T_{9}\right]}{\left\langle T_{1} T_{2} T_{3}^{2}+T_{4} T_{5}+T_{6} T_{7}+T_{8} T_{9}\right\rangle}$ & 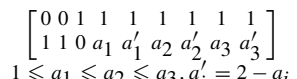 & {$\left[\begin{array}{c}1 \\
a_{3}+1\end{array}\right]$} & 6 \\
\hline
\end{tabular}




\begin{tabular}{|c|c|c|c|c|}
\hline 2 & $\frac{\mathbb{K}\left[T_{1}, \ldots, T_{9}\right]}{\left\langle T_{1} T_{2} T_{3}+T_{4} T_{5}+T_{6} T_{7}+T_{8} T_{9}\right\rangle}$ & 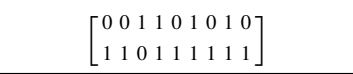 & {$\left[\begin{array}{l}1 \\
2\end{array}\right]$} & 6 \\
\hline 3 & $\frac{\mathbb{K}\left[T_{1}, \ldots, T_{8}\right]}{\left\langle T_{1} T_{2} T_{3}^{2}+T_{4} T_{5}+T_{6} T_{7}+T_{8}^{2}\right\rangle}$ & 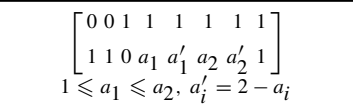 & {$\left[\begin{array}{c}1 \\
a_{2}+1\end{array}\right]$} & 5 \\
\hline 4 & $\begin{array}{c}\frac{\mathbb{K}\left[T_{1}, \ldots, T_{8}, S_{1}, \ldots, S_{m}\right]}{\left\langle T_{1} T_{2}^{l_{2}}+T_{3} T_{4}^{l_{4}}+T_{5} T_{6}^{l_{6}}+T_{7} T_{8}^{l_{8}}\right\rangle} \\
m \geqslant 0 \\
\end{array}$ & $\begin{array}{c}{\left[\begin{array}{cccccccc|ccc}0 & 1 & a_{1} & 1 & a_{2} & 1 & a_{3} & 1 & d_{1} & \ldots & d_{m} \\
1 & 0 & 1 & 0 & 1 & 0 & 1 & 0 & 1 & \ldots & 1\end{array}\right]} \\
0 \leqslant a_{1} \leqslant a_{2} \leqslant a_{3}, d_{1} \leqslant \ldots \leqslant d_{m} \\
l_{2}=a_{1}+l_{4}=a_{2}+l_{6}=a_{3}+l_{8}\end{array}$ & $\begin{array}{c}{\left[\begin{array}{l}d \\
1\end{array}\right]} \\
d \max \\
\text { of } a_{3}, d_{m} \\
\end{array}$ & $m+5$ \\
\hline 5 & $\begin{array}{c}\frac{\mathbb{K}\left[T_{1}, \ldots, T_{8}, S_{1}, \ldots, S_{m}\right]}{\left\langle T_{1} T_{2}+T_{3}^{2} T_{4}+T_{5}^{2} T_{6}+T_{7}^{2} T_{8}\right\rangle} \\
m \geqslant 0\end{array}$ & 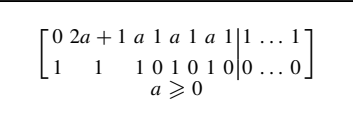 & {$\left[\begin{array}{c}2 a+2 \\
1\end{array}\right]$} & $m+5$ \\
\hline 6 & $\begin{array}{c}\frac{\mathbb{K}\left[T_{1}, \ldots, T_{8}, S_{1}, \ldots, S_{m}\right]}{\left\langle T_{1} T_{2}+T_{3} T_{4}+T_{5}^{2} T_{6}+T_{7}^{2} T_{8}\right\rangle} \\
m \geqslant 0\end{array}$ & 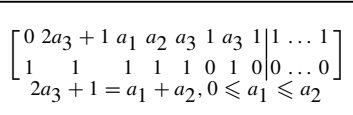 & {$\left[\begin{array}{c}2 a_{3}+2 \\
1\end{array}\right]$} & $m+5$ \\
\hline 7 & $\begin{array}{c}\frac{\mathbb{K}\left[T_{1}, \ldots, T_{8}, S_{1}, \ldots, S_{m}\right]}{\left\langle T_{1} T_{2}+T_{3} T_{4}+T_{5} T_{6}+T_{7}^{2} T_{8}\right\rangle} \\
m \geqslant 1\end{array}$ & 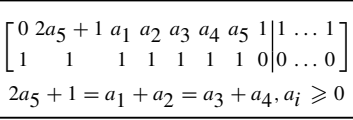 & {$\left[\begin{array}{c}2 a_{5}+2 \\
1\end{array}\right]$} & $m+5$ \\
\hline 8 & $\begin{array}{c}\frac{\mathbb{K}\left[T_{1}, \ldots, T_{8}, S_{1}, \ldots, S_{m}\right]}{\left\langle T_{1} T_{2}+T_{3} T_{4}+T_{5} T_{6}+T_{7} T_{8}\right\rangle} \\
m \geqslant 1\end{array}$ & {$\left[\begin{array}{cccccccc|ccc}0 & 0 & 0 & 0 & 0 & 0 & -1 & 1 & 1 & \ldots & 1 \\
1 & 1 & 1 & 1 & 1 & 1 & 1 & 1 & 0 & \ldots & 0\end{array}\right]$} & {$\left[\begin{array}{l}1 \\
2\end{array}\right]$} & $m+5$ \\
\hline 9 & $\begin{array}{c}\frac{\mathbb{K}\left[T_{1}, \ldots, T_{8}, S_{1}, \ldots, S_{m}\right]}{\left\langle T_{1} T_{2}+T_{3} T_{4}+T_{5} T_{6}+T_{7} T_{8}\right\rangle} \\
m \geqslant 2\end{array}$ & $\begin{array}{c}{\left[\begin{array}{cccccccc|ccc}0 & a_{1} & a_{2} & a_{3} & a_{4} & a_{5} & a_{6} & a_{7} & 1 & \ldots & 1 \\
1 & 1 & 1 & 1 & 1 & 1 & 1 & 1 & 0 & \ldots & 0\end{array}\right]} \\
a_{1}=a_{2}+a_{3}=a_{4}+a_{5}=a_{6}+a_{7} \\
a_{i} \geqslant 0\end{array}$ & {$\left[\begin{array}{c}a_{1}+1 \\
1\end{array}\right]$} & $m+5$ \\
\hline 10 & $\begin{array}{c}\frac{\mathbb{K}\left[T_{1}, \ldots, T_{8}, S_{1}, \ldots, S_{m}\right]}{\left\langle T_{1} T_{2}+T_{3} T_{4}+T_{5} T_{6}+T_{7} T_{8}\right\rangle} \\
m \geqslant 2\end{array}$ & 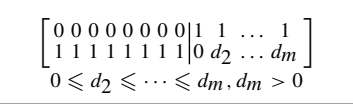 & {$\left[\begin{array}{c}1 \\
d_{m}+1\end{array}\right]$} & $m+5$ \\
\hline 11 & $\begin{array}{c}\frac{\mathbb{K}\left[T_{1}, \ldots, T_{7}, S_{1}, \ldots, S_{m}\right]}{\left\langle T_{1} T_{2}+T_{3} T_{4}+T_{5} T_{6}+T_{7}^{2}\right\rangle} \\
m \geqslant 1\end{array}$ & 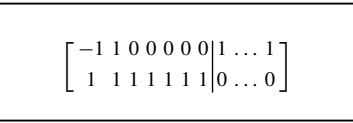 & {$\left[\begin{array}{l}1 \\
2\end{array}\right]$} & $m+4$ \\
\hline 12 & $\begin{array}{c}\frac{\mathbb{K}\left[T_{1}, \ldots, T_{7}, S_{1}, \ldots, S_{m}\right]}{\left\langle T_{1} T_{2}+T_{3} T_{4}+T_{5} T_{6}+T_{7}^{2}\right\rangle} \\
m \geqslant 2\end{array}$ & 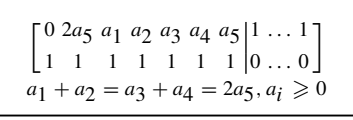 & {$\left[\begin{array}{c}2 a_{5}+1 \\
1\end{array}\right]$} & $m+4$ \\
\hline 13 & $\begin{array}{c}\frac{\mathbb{K}\left[T_{1}, \ldots, T_{7}, S_{1}, \ldots, S_{m}\right]}{\left\langle T_{1} T_{2}+T_{3} T_{4}+T_{5} T_{6}+T_{7}^{2}\right\rangle} \\
m \geqslant 2\end{array}$ & 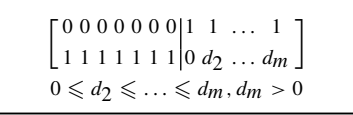 & {$\left[\begin{array}{c}1 \\
d_{m}+1\end{array}\right]$} & $m+4$ \\
\hline 14 & $\begin{array}{c}\mathbb{K}\left[T_{1}, \ldots, T_{10}\right] \\
T_{1} T_{2}+T_{3} T_{4}+T_{5} T_{6}+T_{7} T_{8}, \\
\lambda_{1} T_{3} T_{4}+\lambda_{2} T_{5} T_{6}+T_{7} T_{8}+T_{9} T_{10}\end{array} \mid$ & 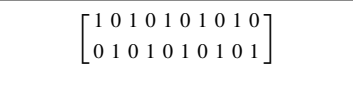 & {$\left[\begin{array}{l}1 \\
1\end{array}\right]$} & 6 \\
\hline
\end{tabular}

Moreover, each of the listed data defines a smooth projective general arrangement variety of true complexity two and Picard number two.

As an application, we contribute to the classification of (almost) Fano varieties, that means varieties with a (semi-)ample anticanonical divisor. Fano varieties show up in the minimal model programme as the general fibers of Mori 
contractions and enjoy remarkable finiteness properties: in the case of suitably mild singularities, they are Mori dream spaces [17, Corollary 1.3.2] and form in any fixed dimension a finite number of families [16]. The classification of smooth Fano varieties has a long history. In dimension one, the projective line $\mathbb{P}_{1}$ is the only example. In dimension two, we have the del Pezzo surfaces, that means $\mathbb{P}_{1} \times \mathbb{P}_{1}$ and the blow ups of the projective plane $\mathbb{P}_{2}$ in at most eight points in general position. The systematic work in the threefold case was initiated by Fano himself in the 1930s and culminated in the classifications given by Iskovskih [49, 50] and Mori/Mukai [59]. From dimension four on, the general classification is widely open.

Here, we consider smooth (almost) Fano varieties that come with a torus action. Existing classification work concerns the toric case $[8,9,54]$ and the case of complexity one [31]. Our Theorem 9.1 classifies in every dimension the (finitely many) smooth Fano general arrangement varieties of complexity two and Picard number two. Moreover, we study the geometry of the resulting Fano varieties. First we provide a raw geometric picture by presenting the (regular) elementary contractions [20], which in the setting of Theorem 9.1 are fibrations and birational contractions of prime divisors onto (possibly singular) Fano varieties of Picard number one; see Remark 9.3. Moreover, also in Remark 9.3, we exhibit small degenerations to varieties with a torus action of true complexity one, where 'small' means that all fibers of the degeneration have the same divisor class group; see [10, Definition 3.1] for a related concept used in a mirror construction. In Remark 9.4, we obtain a similar finiteness feature as observed in [31] in complexity one: all varieties of Theorem 9.1 arise via two elementary contractions and a series of isomorphisms in codimension one from a finite set of smooth projective general arrangement varieties of complexity two having dimension 5 to 8. Finally, we list in Theorem 9.5 the smooth truly almost Fano general arrangement varieties of true complexity two and Picard number two, where truly almost Fano means that the anticanonical divisor is semiample but not ample.

\section{Background on toric varieties and Cox rings}

We provide the necessary background and fix our notation on toric geometry and Cox rings. Throughout the whole article, the ground field $\mathbb{K}$ is algebraically closed and of characteristic zero. Moreover, the word variety refers to an integral separated scheme of finite type over $\mathbb{K}$. In particular, we assume varieties to be irreducible. By a point we mean a closed point.

When we speak of an action of an algebraic group $G$ on a variety $X$, then we always assume the action map $G \times X \rightarrow X,(g, x) \mapsto g \cdot x$ to be a morphism of varieties. A torus is an algebraic group $\mathbb{T}$ isomorphic to a standard torus 
$\mathbb{T}^{n}=\left(\mathbb{K}^{*}\right)^{n}$ and a $\mathbb{T}$-variety is a normal variety $X$ with an effective torus action, where effective means that only the neutral element $\mathbb{1} \in \mathbb{T}$ acts trivially. The complexity $c(X)$ of a $\mathbb{T}$-variety $X$ is the difference $\operatorname{dim}(X)-\operatorname{dim}(\mathbb{T})$.

Toric geometry treats the case of complexity zero. More precisely, a toric variety is a $\mathbb{T}$-variety $Z$ with a base point $z_{0} \in Z$ such that the orbit map $t \mapsto t \cdot z_{0}$ yields an open embedding $\mathbb{T} \rightarrow Z$; we call $\mathbb{T}_{Z}=\mathbb{T}$ the acting torus of $Z$ and write $\mathbb{T}_{Z} \subseteq Z$, identifying $\mathbb{1} \in \mathbb{T}$ with $z_{0} \in Z$ and $\mathbb{T}_{Z}$ with its orbit $\mathbb{T}_{Z} \cdot z_{0}$. Toric geometry originates in Demazure's work [28] in the 1970s and connects combinatorics, represented by fans, with algebraic geometry, represented by toric varieties. As introductory references, we mention [25, 27, 35, 60]. Here comes the fundamental construction, which at the end yields a covariant equivalence between the categories of fans and toric varieties.

Construction 2.1. A fan in $\mathbb{Z}^{n}$ is a finite collection $\Sigma$ of pointed, convex, polyhedral cones living in $\mathbb{Q}^{n}$ such that for any $\sigma \in \Sigma$ also every face of $\sigma$ belongs to $\Sigma$ and for any two $\sigma, \sigma^{\prime} \in \Sigma$ the intersection $\sigma \cap \sigma^{\prime}$ is a face of both, $\sigma$ and $\sigma^{\prime}$. Given a fan $\Sigma$ in $\mathbb{Z}^{n}$, the associated toric variety $Z$ is built by equivariantly gluing the spectra $Z_{\sigma}$ of the monoid algebras $\mathbb{K}\left[M_{\sigma}\right]$ of the monoids $M_{\sigma}:=\sigma^{\vee} \cap \mathbb{Z}^{n}$ of lattice points inside the dual cones:

$$
Z=Z_{\Sigma}=\bigcup_{\sigma \in \Sigma} Z_{\sigma}, \quad Z_{\sigma}=\operatorname{Spec} \mathbb{K}\left[M_{\sigma}\right], \quad \mathbb{K}\left[M_{\sigma}\right]=\bigoplus_{u \in M_{\sigma}} \mathbb{K} \chi^{u}
$$

The acting torus $\mathbb{T}_{Z}=\mathbb{T}^{n}=\operatorname{Spec} \mathbb{K}\left[\mathbb{Z}^{n}\right]$ embeds via $\mathbb{K}\left[M_{\sigma}\right] \subseteq \mathbb{K}\left[\mathbb{Z}^{n}\right]$ canonically into each of the $Z_{\sigma} \subseteq Z$ and one takes the neutral element $\mathbb{1}_{n} \in \mathbb{T}_{Z}=\mathbb{T}^{n}$ as base point $z_{0} \in Z$. The action of $\mathbb{T}_{Z}$ on $Z$ then just extends the group structure of $\mathbb{T}_{Z} \subseteq Z$. Locally, on the affine open subsets $Z_{\sigma} \subseteq Z$, the $\mathbb{T}_{Z}$-action is given by its comorphism $\chi^{u} \mapsto \chi^{u} \otimes \chi^{u}$.

REMARK 2.2. Let $\Sigma$ be a fan in $\mathbb{Z}^{n}$ and $Z$ the associated toric variety. The cones of $\Sigma$ are in bijection with the $\mathbb{T}_{Z}$-orbits via $\sigma \mapsto \mathbb{T}_{Z} \cdot z_{\sigma}$, where $z_{\sigma}$ denotes the common limit point for $t \rightarrow 0$ of all one-parameter groups $t \mapsto\left(t^{v_{1}}, \ldots, t^{v_{n}}\right)$ of $\mathbb{T}_{Z}$ with $v \in \mathbb{Z}^{n}$ taken from the relative interior $\sigma^{\circ} \subseteq \sigma$. The dimension of $\mathbb{T}_{Z} \cdot z_{\sigma}$ equals $n-\operatorname{dim}(\sigma)$. In particular, the rays $\varrho_{1}, \ldots, \varrho_{r}$ of $\Sigma$, that means the one-dimensional cones, define the $\mathbb{T}_{Z}$-invariant prime divisors $D_{i}:=\overline{\mathbb{T}_{Z} \cdot z_{Q_{i}}}$ of $Z$.

Cox's quotient presentation generalizes the classical construction of the projective space $\mathbb{P}_{n}$ as the quotient of $\mathbb{K}^{n+1} \backslash\{0\}$ by $\mathbb{K}^{*}$ acting via scalar multiplication. It delivers, for instance, any complete toric variety as a quotient of an open toric subset of some affine space by a quasitorus, that means an algebraic 
group isomorphic to a direct product of a torus and a finite abelian group. Below and later, we write $\tau \preccurlyeq \sigma$ if $\tau \subseteq \sigma$ is a face of the convex, polyhedral cone $\sigma$.

Construction 2.3. See [24], also [25, Section 5] and [5, Section 2.1.3]. Consider a fan $\Sigma$ in $\mathbb{Z}^{n}$ and let $\varrho_{1}, \ldots, \varrho_{r}$ denote its rays. In each $\varrho_{i}$ sits a unique primitive lattice vector $v_{i}$, the generator of the monoid $\varrho_{i} \cap \mathbb{Z}^{n}$. The generator matrix of $\Sigma$ is the $(n \times r)$-matrix

$$
P=\left[v_{1}, \ldots, v_{r}\right]
$$

having $v_{1}, \ldots, v_{r}$ as its columns, numbered accordingly to $\varrho_{1}, \ldots, \varrho_{r}$. We use the letter $P$ as well to denote the associated linear maps $\mathbb{Z}^{r} \rightarrow \mathbb{Z}^{n}$ and $\mathbb{Q}^{r} \rightarrow \mathbb{Q}^{n}$. As any integral $n \times r$ matrix, $P$ defines a homomorphism of tori

$$
p: \mathbb{T}^{r} \rightarrow \mathbb{T}^{n}, \quad t \mapsto\left(t^{P_{1 *}}, \ldots, t^{P_{n *}}\right)
$$

where $t^{P_{i *}}=t_{1}^{p_{i 1}} \cdots t_{r}^{p_{i r}}$ has the $i$ th row of $P=\left(p_{i j}\right)$ as its exponent vector. Now assume that $v_{1}, \ldots, v_{r}$ generate $\mathbb{Q}^{n}$ as a vector space, meaning that the associated toric variety $Z$ has no torus factor. Consider the orthant $\gamma=\mathbb{Q}_{\geqslant 0}^{r}$ and the set

$$
\hat{\Sigma}:=\{\tau \preccurlyeq \gamma ; P(\tau) \subseteq \sigma \text { for some } \sigma \in \Sigma\} .
$$

Then $\hat{\Sigma}$ is a subfan of the fan $\bar{\Sigma}$ of faces of the orthant $\gamma \subseteq \mathbb{Q}^{r}$. Moreover, $P$ sends cones from $\hat{\Sigma}$ into cones of $\Sigma$. Thus, $p: \mathbb{T}^{r} \rightarrow \mathbb{T}^{n}$ extends to a morphism $p: \hat{Z} \rightarrow Z$ of the associated toric varieties. We arrive at the following picture

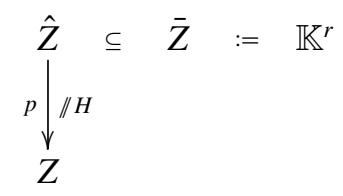

where $\hat{Z} \subseteq \bar{Z}$ is an open $\mathbb{T}^{r}$-invariant subvariety and $H \subseteq \mathbb{T}^{r}$ is the kernel of the homomorphism $p: \mathbb{T}^{r} \rightarrow \mathbb{T}^{n}$ of the acting tori. Being a closed subgroup of a torus, $H$ is a quasitorus. For any cone $\sigma \in \Sigma$, we have

$$
p^{-1}\left(Z_{\sigma}\right)=\hat{Z}_{\hat{\sigma}}, \quad \hat{\sigma}:=\operatorname{cone}\left(e_{i} ; v_{i}=P\left(e_{i}\right) \in \sigma\right), \quad p^{*} \mathcal{O}\left(Z_{\sigma}\right)=\mathcal{O}\left(\hat{Z}_{\hat{\sigma}}\right)^{H},
$$

where $e_{i} \in \mathbb{Z}^{r}$ is the $i$ th canonical basis vector. Thus, $p: \hat{Z} \rightarrow Z$ is an affine morphism and the pull back functions are precisely the $H$-invariants. In other words, $p$ is a good quotient for the $H$-action, as indicated by ' $/ / H$ '.

Generalizing the idea of homogeneous coordinates on the projective space, one uses Cox's quotient presentation to obtain global coordinates on toric varieties. 
REMARK 2.4. Let $Z$ be a toric variety with quotient presentation $p: \hat{Z} \rightarrow Z$ as in Construction 2.3. Then every $p$-fiber contains a unique closed $H$-orbit. The presentation in Cox coordinates of a point $x \in Z$ is

$$
x=\left[z_{1}, \ldots, z_{r}\right], \quad \text { where } z=\left(z_{1}, \ldots, z_{r}\right) \in p^{-1}(x) \text { with } H \cdot z \subseteq \hat{Z} \text { closed } .
$$

Thus, $[z]$ and $\left[z^{\prime}\right]$ represent the same point $x \in Z$ if and only if $z$ and $z^{\prime}$ lie in the same closed $H$-orbit of $\hat{Z}$. For instance, the points $z_{\sigma} \in Z$, where $\sigma \in \Sigma$, are given in Cox coordinates as

$$
z_{\sigma}=\left[\varepsilon_{1}, \ldots, \varepsilon_{r}\right], \quad \varepsilon_{i}= \begin{cases}0, & P\left(e_{i}\right) \in \sigma, \\ 1, & P\left(e_{i}\right) \notin \sigma .\end{cases}
$$

REMARK 2.5. Let $Z$ be a toric variety with quotient presentation $p: \hat{Z} \rightarrow Z$ as in Construction 2.3. Then we obtain an injection from the closed subvarieties $X \subseteq Z$ to the $H$-invariant closed subvarieties of $\bar{Z}=\mathbb{K}^{r}$ via

$$
X \mapsto \bar{X}:=\overline{p^{-1}(X)} \subseteq \bar{Z}
$$

The vanishing ideal $I(\bar{X}) \subseteq \mathbb{K}\left[T_{1}, \ldots, T_{r}\right]$ is generated by polynomials $g_{1}, \ldots$, $g_{s}$ being $H$-homogeneous in the sense that $g_{j}(h \cdot z)=\chi_{j}(h) g_{j}(z)$ holds with characters $\chi_{j} \in \mathbb{X}(H)$. We call $g_{1}, \ldots, g_{s}$ defining equations in Cox coordinates for $X \subseteq Z$.

We turn to Cox rings. Their history starts in the 1970s in a geometric setting, when Colliot-Thélène and Sansuc introduced the universal torsors presenting smooth varieties in a universal way as quotients [23]. In toric geometry, the quotient presentation and Cox rings popped up in the 1990s in the work of Audin [6], Cox [24] and others. In 2000, Hu and Keel observed fundamental connections between Cox rings, Mori theory and geometric invariant theory [48]. As a general introductory reference on Cox rings, we mention [5].

We enter the subject. Consider a normal variety $X$ with only constant invertible global functions and finitely generated divisor class group $\mathrm{Cl}(X)$. For a Weil divisor $D$ on $X$, denote by $\mathcal{O}(D)$ the associated sheaf of sections. Then the Cox sheaf $\mathcal{R}$ and the Cox ring $\mathcal{R}(X)$ of $X$ are defined as

$$
\mathcal{R}:=\bigoplus_{[D] \in \mathrm{Cl}(X)} \mathcal{O}(D), \quad \mathcal{R}(X):=\Gamma(X, \mathcal{R})=\bigoplus_{[D] \in \mathrm{Cl}(X)} \Gamma(X, \mathcal{O}(D))
$$

Observe that we grade $\mathcal{R}$ and $\mathcal{R}(X)$ by divisor classes, whereas the homogeneous components are defined by divisors. If $\mathrm{Cl}(X)$ is torsion free, then this problem 
of well-definedness is solved by just regarding $\mathcal{R}$ as the sheaf of multi-section algebras: fix a subgroup $K \subseteq \operatorname{WDiv}(X)$ of the Weil divisor group mapping isomorphically onto $\mathrm{Cl}(X)$ and work with

$$
\mathcal{R}:=\mathcal{S}=\bigoplus_{D \in K} \mathcal{O}(D)
$$

The case of torsion in $\mathrm{Cl}(X)$ requires more care: fix a subgroup $K \subseteq \operatorname{WDiv}(X)$ mapping onto $\mathrm{Cl}(X)$, denote by $K^{0} \subseteq K$ the subgroup consisting of all principal divisors of $K$ and choose functions $\chi^{E} \in \mathbb{K}(X)$, where $E \in K^{0}$, satisfying

$$
\operatorname{div}\left(\chi^{E}\right)=E, \quad \chi^{E} \chi^{E^{\prime}}=\chi^{E+E^{\prime}} .
$$

Consider the sheaf of $\mathcal{S}$ multi-section algebras associated with $K$, the subsheaf $\mathcal{I} \subseteq \mathcal{S}$ of ideals generated by $1-\chi^{E}$, where $E \in K^{0}$ and define $\mathcal{R}:=\mathcal{S} / \mathcal{I}$. Then $\mathcal{R}$ is graded by $K / K^{0}=\mathrm{Cl}(X)$ via

$$
\mathcal{R}_{[D]}:=\pi\left(\bigoplus_{D+K^{0}} \mathcal{O}(D)\right)
$$

where $\pi: \mathcal{S} \rightarrow \mathcal{R}$ denotes the projection. Then, up to isomorphy, this construction turns out not to depend on any of the choices made; we refer to $[14,40]$ and [5, Section 1.1.4] for the details.

Remark 2.6. See [24], also [25, Section 5] and [5, Section 2.1.3]. Let $Z$ be a toric variety without torus factor and let $D_{1}, \ldots, D_{r}$ be the $\mathbb{T}_{Z}$-invariant prime divisors of $Z$. Then the Cox ring of $Z$ and its $\mathrm{Cl}(Z)$-grading are given as

$$
\mathcal{R}(Z)=\mathbb{K}\left[T_{1}, \ldots, T_{r}\right], \quad \operatorname{deg}\left(T_{i}\right)=\left[D_{i}\right] \in \mathrm{Cl}(Z) .
$$

For a more explicit picture, let $Z$ arise from a fan $\Sigma$ in $\mathbb{Z}^{n}$. Then $D_{i}=\overline{\mathbb{T}_{Z} \cdot z_{\varrho_{i}}} \subseteq Z$ holds with the rays $\varrho_{1}, \ldots, \varrho_{r}$ of $\Sigma$. The divisor class group of $Z$ and the divisor classes of the $D_{i}$ are described by

$$
\mathrm{Cl}(Z)=K:=\mathbb{Z}^{r} / \operatorname{im}\left(P^{*}\right), \quad\left[D_{i}\right]=w_{i}:=Q\left(e_{i}\right) \in K,
$$

where we denote by $P^{*}$ the transpose of the generator matrix $P$ of $\Sigma$, by $Q: \mathbb{Z}^{r} \rightarrow$ $K$ the projection and by $e_{i} \in \mathbb{Z}^{r}$ the $i$ th canonical basis vector.

REMARK 2.7. In Construction 2.3, the toric variety $Z$ is represented as a quotient of $\hat{Z} \subseteq \mathbb{K}^{r}$ by the quasitorus $H=\operatorname{ker}(p) \subseteq \mathbb{T}^{r}$. With $K=\mathrm{Cl}(Z)=\mathbb{Z}^{r} / \operatorname{im}\left(P^{*}\right)$ 
from Remark 2.6, we can view $H$ also as the spectrum of the associated group algebra:

$$
H=\operatorname{Spec} \mathbb{K}[K], \quad \mathbb{K}[K]=\bigoplus_{w \in K} \mathbb{K} \chi^{w} .
$$

Here, the elements $\chi^{w} \in \mathbb{K}[K]$ are the characters of $H$ and $w \mapsto \chi^{w}$ defines an isomorphism between $K$ and the character group $\mathbb{X}(H)$. Setting $\chi_{i}:=\chi^{w_{i}}$, we retrieve the $H$-action from the $\mathrm{Cl}(Z)$-grading of the Cox $\operatorname{ring} \mathcal{R}(Z)$ as

$$
h \cdot z=\left(\chi_{1}(h) z_{1}, \ldots, \chi_{r}(h) z_{r}\right) .
$$

In general, the Cox ring $\mathcal{R}(X)$ is normal, integral and, as its main algebraic feature, it is $\mathrm{Cl}(X)$-factorial $[4,40]$. Let us recall the meaning. A ring $R=\oplus_{K} R_{w}$ graded by an abelian group $K$ is $K$-integral if it has no homogeneous zero divisors. A nonzero homogeneous nonunit $f \in R$ is $K$-prime if, whenever $f$ divides a product $g h$ of two homogeneous $g, h \in R$, then it divides $g$ or $h$. The ring $R$ is called $K$-factorial if it is $K$-integral and every nonzero homogeneous nonunit of $R$ is a product of $K$-primes. If $\mathrm{Cl}(X)$ is torsion free, then the Cox ring admits unique factorization in the usual sense; see [14, Proposition 8.4] and also [29, Corollary 1.2].

The bunched ring approach presented in $[5,15,39]$ uses Cox rings to encode algebraic varieties. The central construction starts with a given $K$-factorial ring $R$ and produces varieties $X$ having divisor class group $K$ and Cox ring $R$. In this article, we will work with the following variant being closer to toric geometry in the sense that it uses fans instead of the bunches of cones of $[5,15,39]$. Let us fix the necessary notation. By an affine algebra we mean a finitely generated reduced $\mathbb{K}$-algebra. If $K$ is an abelian group, then we denote by $K_{\mathbb{Q}}=K \otimes_{\mathbb{Z}} \mathbb{Q}$ the associated rational vector space. Given $w \in K$, we write as well $w$ for the element $w \otimes 1 \in K_{\mathbb{Q}}$. Moreover, if $Q: K \rightarrow K^{\prime}$ is a homomorphism, we denote the associated linear map $K_{\mathbb{Q}} \rightarrow K_{\mathbb{Q}}^{\prime}$ as well by $Q$.

CONSTRUCTION 2.8. Let $K$ be a finitely generated abelian group and $R=$ $\oplus_{K} R_{w}$ a $K$-factorial, normal, integral, affine $\mathbb{K}$-algebra with only constant homogeneous units. Suppose that $f_{1}, \ldots, f_{r}$ are pairwise nonassociated $K$-prime generators of $R$ such that any $r-1$ of the degrees $w_{i}:=\operatorname{deg}\left(f_{i}\right)$ generate $K$ as a group and for $\tau_{i}:=\operatorname{cone}\left(w_{j} ; j \neq i\right)$, the intersection $\tau_{1} \cap \cdots \cap \tau_{r}$ is of full dimension in $K_{\mathbb{Q}}$. Consider the closed embedding

$$
\operatorname{Spec} R=: \bar{X} \stackrel{x \mapsto\left(f_{1}(x), \ldots, f_{r}(x)\right)}{\longrightarrow} \bar{Z}:=\mathbb{K}^{r} .
$$

The quasitorus $H=\operatorname{Spec} \mathbb{K}[K]$ acts on $\bar{Z}$ via $h \cdot z=\left(\chi_{1}(h) z_{1}, \ldots, \chi_{r}(h) z_{r}\right)$, where $\chi_{i} \in \mathbb{X}(H)$ is the character corresponding to $w_{i} \in K$. This action leaves 
the subvariety $\bar{X} \subseteq \bar{Z}$ invariant. Now, consider the degree map

$$
Q: \mathbb{Z}^{r} \rightarrow K, \quad e_{i} \mapsto w_{i}
$$

Let $P$ be an integral $(n \times r)$-matrix, the rows of which generate $\operatorname{ker}(Q) \subseteq \mathbb{Z}^{r}$. Then the assumptions on $f_{1}, \ldots, f_{r}$ ensure that the columns of $P$ are pairwise different and primitive; see [5, Theorem 2.2.2.6 and Lemma 2.1.4.1]. Fix any fan $\Sigma$ having $P$ as generator matrix. The associated toric variety $Z$ and $p: \hat{Z} \rightarrow Z$ from Construction 2.3 fit into a commutative diagram

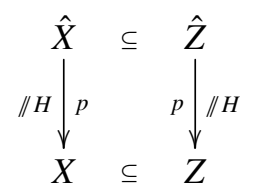

where we set $\hat{X}:=\bar{X} \cap \hat{Z}$ and $X:=\hat{X} / / H$ is a normal, closed subvariety of $Z$. We speak of $X \subseteq Z$ as an explicit variety, refer to $\alpha=\left(f_{1}, \ldots, f_{r}\right)$ as the embedding system of $X \subseteq Z$ and call any system of $K$-homogeneous generators $g_{1}, \ldots, g_{s}$ of the vanishing ideal $I(\bar{X}) \subseteq \mathbb{K}\left[T_{1}, \ldots, T_{r}\right]$ defining equations for $X$.

REMARK 2.9. If, in Construction 2.8, the ambient toric variety $Z$ is affine (complete, projective), then the resulting $X$ is affine (complete, projective).

The interface from explicit varieties to bunched rings relies on linear Gale duality. Here comes how this concretely works in our situation.

REMARK 2.10. Notation as in Construction 2.3, 2.6 and Construction 2.8. To any explicit variety $X \subseteq Z$, we can apply the machinery of bunched rings [5, Ch. 3]. The translation into the latter setting runs as follows. Consider the homomorphisms

$$
P: \mathbb{Z}^{r} \rightarrow \mathbb{Z}^{n}, e_{i} \mapsto v_{i}, \quad Q: \mathbb{Z}^{r} \rightarrow K, e_{i} \mapsto w_{i},
$$

where $K=\mathbb{Z}^{r} / \operatorname{im}\left(P^{*}\right)$. For $\sigma \in \Sigma$, the face $\hat{\sigma} \preccurlyeq \gamma \subseteq \mathbb{Q}^{r}$ of the orthant is generated by the $e_{i}$ with $v_{i} \in \sigma$. The complementary face $\hat{\sigma}^{*} \preccurlyeq \gamma$ of $\hat{\sigma} \preccurlyeq \gamma$ is generated by the $e_{i}$ with $e_{i} \notin \hat{\sigma}$. Set

$$
\Phi:=\left\{Q\left(\hat{\sigma}^{*}\right) ; \sigma \in \Sigma \text { with } X \cap \mathbb{T}_{z} \cdot z_{\sigma} \neq \emptyset\right\} .
$$

Then $\Phi$ is a collection of cones in $K_{\mathbb{Q}}$ with pairwise intersecting relative interiors. With the system of generators $\mathfrak{F}:=\left(f_{1}, \ldots, f_{r}\right)$, we obtain a bunched ring $(R, \mathfrak{F}$, $\Phi)$ in the sense of [5, Definition 3.2.1.2]. We have an open inclusion

$$
X \subseteq X(R, \mathfrak{F}, \Phi)
$$


into the variety associated with the bunched ring [5, Definition 3.2.1.3] such that the complement of $X$ in $X(R, \mathfrak{F}, \Phi)$ is of codimension at least two. If $X$ is affine or complete, then the above inclusion is even an equality.

Based on this translation, we will import several statements on the geometry of explicit ( $\mathbb{T}$-)varieties in Section 5. For the moment, we just mention the following.

REMARK 2.11. See [5, Theorem 3.2.1.4]. For every explicit variety $X \subseteq Z$, the divisor class group and the Cox ring of $X$ are given as

$$
\mathrm{Cl}(X)=K=\mathrm{Cl}(Z), \quad \mathcal{R}(X)=R=\mathcal{R}(Z) / I(\bar{X}) .
$$

Moreover, $\hat{X}$ is the relative spectrum of the Cox sheaf on $X$, which in turn is given as the direct image $\mathcal{R}=p_{*} \mathcal{O}_{\hat{X}}$. Finally, we have the prime divisors

$$
D_{i}^{X}=X \cap D_{i}^{Z} \subseteq X
$$

induced by the toric prime divisors $D_{1}^{Z}, \ldots, D_{r}^{Z}$. Here each $D_{i}^{X}$ is determined by the property $p^{*} D_{i}^{X}=V_{\bar{X}}\left(T_{i}\right)$.

Coming embedded into a toric variety, every explicit variety $X \subseteq Z$ inherits the $A_{2}$-property: any two points of $X$ admit a common affine neighborhood. The normal $A_{2}$-varieties are precisely the normal varieties that are embeddable into a toric variety; see [69]. An $A_{2}$-variety $Y$ is $A_{2}$-maximal if it does not allow open embeddings into $A_{2}$-varieties $Y^{\prime}$ such that $Y^{\prime} \backslash Y$ is nonempty of codimension at least two. For example, affine and projective varieties are $A_{2}$-maximal.

REMARK 2.12. See [5, Theorem 3.2.1.9]. Every $A_{2}$-maximal variety with only constant invertible global functions, finitely generated divisor class group and finitely generated Cox ring can be represented as an explicit variety.

In [48], Hu and Keel introduced the Mori dream spaces as $\mathbb{Q}$-factorial projective varieties with a Mori chamber decomposition satisfying suitable finiteness properties which in particular guarantee an optimal behavior with respect to the minimal model programme.

REMARK 2.13. According to [48, Proposition 2.9], the Mori dream spaces are precisely the $\mathbb{Q}$-factorial projective varieties with a finitely generated Cox ring. In particular, every Mori dream space can be represented as an explicit variety. 


\section{Constructing explicit $\mathbb{T}$-varieties}

We present our method of producing systematically explicit $\mathbb{T}$-varieties, see Construction 3.5, and we formulate basic properties, see Proposition 3.7, Theorem 3.10 and Proposition 3.16. The proofs of the latter results are given in the subsequent section. We begin by indicating the ideas behind Construction 3.5. First, take a glance at the following naive way to produce varieties with torus action sitting inside a given toric variety.

RECIPE 3.1. Let $Z$ be a toric variety, $\mathbb{T}_{Z}=\mathbb{T}^{\prime} \times \mathbb{T}$ a splitting of the acting torus into closed subtori and $Y \subseteq \mathbb{T}^{\prime}$ a closed subvariety. Consider the closure

$$
X:=\overline{Y \times \mathbb{T}} \subseteq Z
$$

Then the variety $X \subseteq Z$ is invariant under the action of $\mathbb{T}$ on $Z$ and thus we obtain an effective algebraic torus action $\mathbb{T} \times X \rightarrow X$. By construction, we have

$$
X \cap \mathbb{T}_{Z}=Y \times \mathbb{T}, \quad \mathbb{K}(X)^{\mathbb{T}}=\mathbb{K}(Y) .
$$

In particular, $Y$ represents the field of $\mathbb{T}$-invariant rational functions of $X$ and thus the projection $\mathbb{T}_{Z} \rightarrow \mathbb{T}^{\prime}$ defines a rational quotient $X \rightarrow Y$ for the $\mathbb{T}$-action on $X$.

So far, Recipe 3.1 provides no specifically close relations between the geometry of $X$ and that of its ambient toric variety $Z$. Nevertheless, we know in advance the rational quotient $Y$ and, stemming from a subtorus action on $Z$, the $\mathbb{T}$-action on $X$ can be studied by toric methods. Moreover, Recipe 3.1 produces for instance all projective $\mathbb{T}$-varieties, as we infer from the following.

REMARK 3.2. Any $\mathbb{T}$-variety $X$ that admits an equivariant embedding into a toric variety $Z$ with $\mathbb{T}$ acting as a subtorus of $\mathbb{T}_{Z}$ can be represented as in Recipe 3.1. The techniques from $[38,39]$ yield such equivariant embeddings for $\mathbb{T}$-varieties $X$ with the $A_{2}$-property provided they are $\mathbb{Q}$-factorial or, more generally, divisorial in the sense of [18], or have a Cox sheaf of locally finite type.

Our aim is to bring together the features of Recipe 3.1 with those of Construction 2.8. Let us first look at a concrete example, indicating the main rules of the subsequent construction game and illustrating the notation used there. The example we are going to treat is a well-known $\mathbb{K}^{*}$-surface, occurring as an important step in resolving the $E_{6}$-singular cubic surface; see [37, Section 4] and, for links to various other aspects, also [5, page 522]. 
EXAMPLE 3.3. Our initial data is a projective line $Y \subseteq \mathbb{P}_{2}$ given in homogeneous coordinates by the following equation:

$$
Y=V\left(T_{0}+T_{1}+T_{2}\right) \subseteq \mathbb{P}_{2} .
$$

We regard $\mathbb{P}_{2}$ as the toric variety defined by the complete fan $\Delta$ with the generator matrix

$$
B=\left[u_{0}, u_{1}, u_{2}\right]=\left[\begin{array}{lll}
-1 & 1 & 0 \\
-1 & 0 & 1
\end{array}\right] .
$$

Now we start the game that builds up the generator matrix $P$ of the fan of the prospective ambient toric variety $Z$ of our final $X$. First produce a matrix

$$
P_{0}=\left[u_{01}, u_{02}, u_{11}, u_{21}\right]=\left[\begin{array}{llll}
-3 & -1 & 3 & 0 \\
-3 & -1 & 0 & 2
\end{array}\right]
$$

the columns $u_{i j}$ of which are positive multiples of the columns $u_{i}$ of $B$. Then append a zero column to $P_{0}$, a block $d$ below $P_{0}$ and a block $d^{\prime}$ below the zero column:

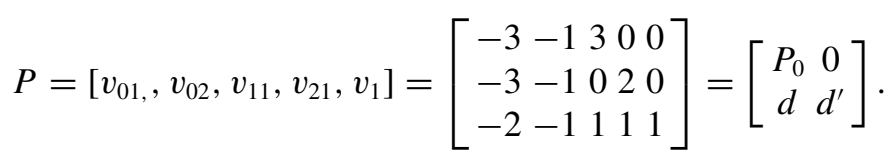

Let $\Sigma$ be any complete fan in $\mathbb{Z}^{3}$ having $P$ as its generator matrix and let $Z$ be the associated toric variety. Then the acting torus $\mathbb{T}_{Z}$ of $Z$ splits as

$$
\mathbb{T}_{Z}=\mathbb{T}^{3}=\mathbb{T}^{2} \times \mathbb{K}^{*}
$$

Moreover, $Y \cap \mathbb{T}^{2} \subseteq \mathbb{T}^{2}$ is the zero set of $1+T_{1} / T_{0}+T_{2} / T_{0}$. Proceeding exactly as in Recipe 3.1 yields a surface $X$ coming with an effective $\mathbb{K}^{*}$-action:

$$
X:=\overline{\left(Y \cap \mathbb{T}^{2}\right) \times \mathbb{K}^{*}} \subseteq Z .
$$

We have $X \cap \mathbb{T}^{3}=V\left(1+T_{1} / T_{0}+T_{2} / T_{0}\right)$. Pulling back that equation via the homomorphism $p: \mathbb{T}^{5} \rightarrow \mathbb{T}^{3}$ given by $P$ leads to the equation for $X$ in Cox coordinates:

$$
\bar{X}=V\left(T_{01}^{3} T_{02}+T_{11}^{3}+T_{21}^{2}\right) \subseteq \bar{Z}=\mathbb{K}^{5},
$$

where the variables $T_{i j}$ represent columns of $P_{0}$ and the index $i j$ tells us that we have the $j$ th repetition of the $i$ th column of $B$, scaled by the exponent $l_{i j}$ of $T_{i j}$.

REMARK 3.4. In Example 3.3, we encountered two explicit varieties in the sense of Construction 2.8: first, the projective line $Y \subseteq \mathbb{P}_{2}$ and second, the $\mathbb{K}^{*}$-surface $X \subseteq Z$. In particular, divisor class group and Cox ring of $X$ are given as

$$
\mathrm{Cl}(X)=K=\mathbb{Z}^{5} / \operatorname{im}\left(P^{*}\right)=\mathbb{Z}^{2},
$$




$$
\mathcal{R}(X)=\mathbb{K}\left[T_{01}, T_{02}, T_{11}, T_{21}, T_{1}\right] /\left\langle T_{01}^{3} T_{02}+T_{11}^{3}+T_{21}^{2}\right\rangle .
$$

Observe that the manipulations on the matrix $B$ turned the redundant defining relation $T_{0}+T_{1}+T_{2}$ of $Y$ into the serious relation $T_{01}^{3} T_{02}+T_{11}^{3}+T_{21}^{2}$, defining the resulting $X$.

We come to the general construction of explicit $\mathbb{T}$-varieties. It starts with a given explicit variety $Y \subseteq Z_{\Delta}$ provided by Construction 2.8 and delivers an explicit variety $X \subseteq Z_{\Sigma}$ which is invariant under a direct factor $\mathbb{T} \subseteq \mathbb{T}_{\Sigma}$ of the acting torus $\mathbb{T}_{\Sigma} \subseteq Z_{\Sigma}$.

CONSTRUCTION 3.5. Let $Y \subseteq Z_{\Delta}$ be an explicit variety with embedding system $\alpha=\left(f_{0}, \ldots, f_{r}\right)$. The defining fan $\Delta$ of $Z_{\Delta}$ lives in some $\mathbb{Z}^{t}$ and has a $t \times(r+1)$ generator matrix

$$
B=\left[u_{0}, \ldots, u_{r}\right] .
$$

In particular, $\mathrm{Cl}(Y)=\mathrm{Cl}\left(Z_{\Delta}\right)$ equals $K_{B}:=\mathbb{Z}^{r+1} / \operatorname{im}\left(B^{*}\right)$ and the Cox ring of $Y$ equals the $K_{B}$-factorial input ring $R_{Y}$ of Construction 2.8. We build up a new matrix from $B$ and the following data

- positive integers $n_{0}, \ldots, n_{r}$ and non-negative integers $m, s$ with $t+s \leqslant n+m$, where $n:=n_{0}+\cdots+n_{r}$,

- for any two $i, j$, where $i=0, \ldots, r$ and $j=1, \ldots, n_{i}$, a positive integer $l_{i j}$ and a vector $d_{i j} \in \mathbb{Z}^{s}$,

- for any $k$, where $1 \leqslant k \leqslant m$, a vector $d_{k}^{\prime} \in \mathbb{Z}^{s}$, where, with the multiples $u_{i j}:=l_{i j} u_{i} \in \mathbb{Z}^{t}$ of the columns of $B$, we require that the vectors

$$
v_{i j}=\left(u_{i j}, d_{i j}\right) \in \mathbb{Z}^{t+s}, \quad v_{k}=\left(0, d_{k}^{\prime}\right) \in \mathbb{Z}^{t+s}
$$

are all primitive, any two of them are distinct and altogether they generate $\mathbb{Q}^{t+s}$ as a vector space. Store $v_{i j}$ and $v_{k}$ as columns in a $(t+s) \times(n+m)$ matrix

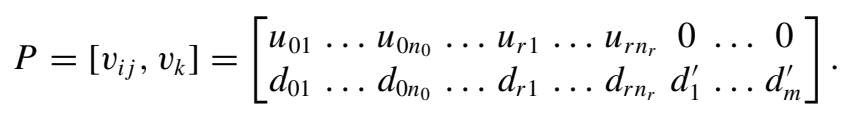

Now, let $\Sigma$ be any fan in $\mathbb{Z}^{t+s}$ having $P$ as its generator matrix and denote by $Z_{\Sigma}$ the associated toric variety. Then we obtain a commutative diagram

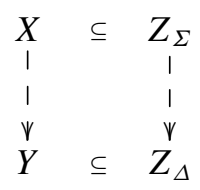


where the rational map $Z_{\Sigma} \rightarrow Z_{\Delta}$ is given by the projection $\mathbb{T}^{t} \times \mathbb{T}^{s} \rightarrow \mathbb{T}^{t}$ of the respective acting tori $\mathbb{T}_{\Sigma}=\mathbb{T}^{t} \times \mathbb{T}^{s}$ and $\mathbb{T}_{\Delta}=\mathbb{T}^{t}$ and we define

$$
X:=X(\alpha, P, \Sigma):=\overline{\left(Y \cap \mathbb{T}^{t}\right) \times \mathbb{T}^{s}} \subseteq Z_{\Sigma} .
$$

Then $X \subseteq Z_{\Sigma}$ is invariant under the action of the subtorus $\mathbb{T}=\left\{\mathbb{1}_{t}\right\} \times \mathbb{T}^{s}$ of the acting torus $\mathbb{T}_{\Sigma}=\mathbb{T}^{t} \times \mathbb{T}^{s}$ of $Z_{\Sigma}$. Moreover, set

$$
T_{i}^{l_{i}}:=T_{i 1}^{l_{i 1}} \cdots T_{i n_{i}}^{l_{i n_{i}}} \in \mathbb{K}\left[T_{i j}, S_{k}\right], \quad K_{P}:=\mathbb{Z}^{n+m} / \operatorname{im}\left(P^{*}\right)=\operatorname{Cl}\left(Z_{\Sigma}\right) .
$$

Let $h_{1}, \ldots, h_{q}$ be defining equations of $Y$ in Cox coordinates, that means $K_{B^{-}}$ homogeneous generators for the ideal of relations between $f_{0}, \ldots, f_{r}$. Consider the factor ring

$$
R(\alpha, P):=\mathbb{K}\left[T_{i j}, S_{k}\right] /\left\langle h_{1}\left(T_{0}^{l_{0}}, \ldots, T_{r}^{l_{r}}\right), \ldots, h_{q}\left(T_{0}^{l_{0}}, \ldots, T_{r}^{l_{r}}\right)\right\rangle
$$

and denote by $Q_{P}: \mathbb{Z}^{n+m} \rightarrow K_{P}$ the projection. We turn $R(\alpha, P)$ into a $K_{P^{-}}$ graded algebra via

$$
\operatorname{deg}\left(T_{i j}\right):=w_{i j}:=Q_{P}\left(e_{i j}\right), \quad \operatorname{deg}\left(T_{k}\right):=w_{k}:=Q_{P}\left(e_{k}\right),
$$

where $e_{i j}, e_{k} \in \mathbb{Z}^{n+m}$ are the canonical basis vectors. Observe that we have a unique homomorphism of graded algebras $R_{Y} \rightarrow R(\alpha, P)$ sending $f_{i}$ to $T_{i}^{l_{i}}$.

REMARK 3.6. For $t=n=0$, the above construction yields the usual construction of a toric variety from a fan. Moreover, for $s=m=0$ and $n=r+1$, we arrive at Construction 2.8.

We come to the first basic property of Construction 3.5. Note that in concrete cases the assumptions of this proposition on $R(\alpha, P)$ and the $T_{i j}$ made below can be checked algorithmically via absolute factorization; see [44, Remark 3.8].

Proposition 3.7. Let $X=X(\alpha, P, \Sigma)$ arise from Construction 3.5. If $R(\alpha, P)$ is a $K_{P}$-integral affine algebra with only constant homogeneous units and the $T_{i j}$ define pairwise nonassociated $K_{P}$-primes in $R(\alpha, P)$, then $X \subseteq Z_{\Sigma}$ is an explicit variety.

Definition 3.8. By an explicit $\mathbb{T}$-variety $X \subseteq Z$ we mean a variety $X=X(\alpha$, $P, \Sigma)$ in $Z=Z_{\Sigma}$ together with the action of $\mathbb{T}=\left\{\mathbb{1}_{t}\right\} \times \mathbb{T}^{s}$ arising from Construction 3.5 such that the assumptions of Proposition 3.7 are satisfied.

COROLlary 3.9. Let $X \subseteq Z$ be an explicit $\mathbb{T}$-variety. Then $X$ is a normal variety with only constant invertible global functions. Moreover, dimension, complexity, 
divisor class group and Cox ring of $X$ are given by

$\operatorname{dim}(X)=s+\operatorname{dim}(Y), \quad c(X)=\operatorname{dim}(Y), \quad \mathrm{Cl}(X)=K_{P}, \quad \mathcal{R}(X)=R(\alpha, P)$.

We say that a $\mathbb{T}$-variety $X^{\prime}$ admits a presentation as an explicit $\mathbb{T}$-variety if there is a $\mathbb{T}$-equivariant isomorphism $X^{\prime} \rightarrow X$ with some explicit $\mathbb{T}$-variety $X \subseteq Z$.

THEOREM 3.10. Let $X$ be an $A_{2}$-maximal $\mathbb{T}$-variety having only constant invertible global functions, finitely generated divisor class group and finitely generated Cox ring. Then $X$ admits a presentation as an explicit $\mathbb{T}$-variety.

In the rest of the section, we discuss the geometry of the torus action of an explicit $\mathbb{T}$-variety $X \subseteq Z$, aiming for a suitable quotient. First, we continue Example 3.3.

EXAMPLE 3.11. Consider again the explicit $\mathbb{K}^{*}$-surface $X \subseteq Z$ from 3.3. An important source of information is the location of the columns of $P$ over those of $B$ with respect to the projection pr: $\mathbb{Z}^{3} \rightarrow \mathbb{Z}^{2}$ onto the first two coordinates:

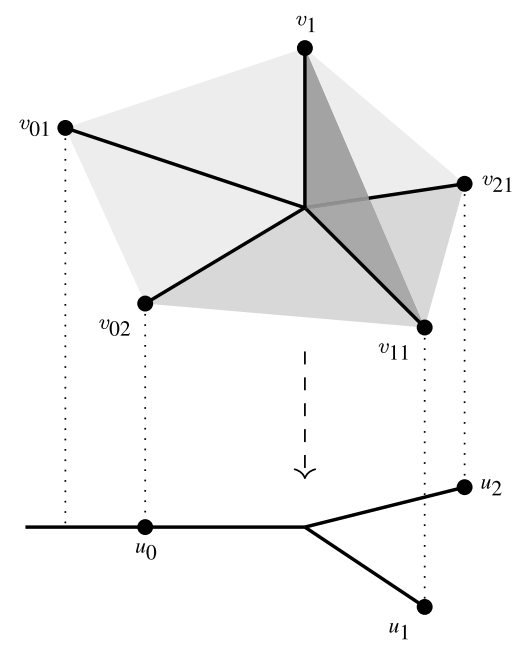

Each column $v_{i j}$ projects into the ray through $u_{i}$ and $v_{1}$ lies in the kernel of the projection. The rays $\varrho_{i j}$ through $v_{i j}$ and $\varrho_{1}$ through $v_{1}$ define prime divisors $D_{i j}^{Z}$ and $E_{1}^{Z}$ of $Z$, respectively. Cutting down to $X$ gives us prime divisors

$$
D_{i j}^{X}:=X \cap D_{i j}^{Z} \subseteq X, \quad E_{1}^{X}:=X \cap E_{1}^{Z} \subseteq X,
$$

where the basic reason for primality is that the divisors are given in Cox coordinates by $K$-prime ideals; for instance $D_{01}$ is defined by $\left\langle T_{01}, T_{11}^{3}+T_{21}^{2}\right\rangle$. 
We are interested in the isotropy groups. Recall that $\mathbb{K}^{*}$ acts on $X$ as the subtorus

$$
\mathbb{T}:=\left\{\mathbb{1}_{2}\right\} \times \mathbb{K}^{*} \subseteq \mathbb{T}^{3}=\mathbb{T}_{Z}
$$

In particular, the isotropy groups of the $\mathbb{T}$-action are constant along the $\mathbb{T}_{Z}$-orbits. Consider the kernel $L=\{0\} \times \mathbb{Z}$ of pr: $\mathbb{Z}^{3} \rightarrow \mathbb{Z}^{2}$. Then [5, Proposition 2.1.4.2] yields for any $\sigma \in \Sigma$ that the isotropy group of $\mathbb{T}$ at $z_{\sigma} \in Z$ has character group

$$
\mathbb{X}\left(\mathbb{T}_{z_{\sigma}}\right) \cong(L \cap \operatorname{lin}(\sigma)) \oplus\left(\operatorname{pr}(\operatorname{lin}(\sigma)) \cap \mathbb{Z}^{2}\right) /\left(\operatorname{pr}\left(\operatorname{lin}(\sigma) \cap \mathbb{Z}^{3}\right),\right.
$$

where $\operatorname{lin}(\sigma) \subseteq \mathbb{Q}^{3}$ denotes the $\mathbb{Q}$-linear hull. Looking at $\sigma=\varrho_{i j}$, we see that the isotropy group $\mathbb{T}_{x}$ of the general point $x \in D_{i j}^{X}$ is cyclic of order $l_{i j}$, where $l_{i j}$ is the exponent of $T_{i j}$ in the defining relation of $X$, that means

$$
l_{01}=3, \quad l_{02}=1, \quad l_{11}=3, \quad l_{21}=2 .
$$

Moreover, the curve $E_{1}^{X}$ consists of fixed points of the $\mathbb{T}$-action and there are two isolated fixed points, forming the intersections of $X$ with the toric orbits $\mathbb{T}_{Z} \cdot z_{\sigma}$ for $\sigma=\operatorname{cone}\left(v_{01}, v_{02}\right)$ and $\sigma=\operatorname{cone}\left(v_{02}, v_{11}, v_{21}\right)$, respectively. In particular, we see that

$$
X_{0}=X \cap\left(\mathbb{T}_{Z} \cup \mathbb{T}_{Z} \cdot z_{\varrho_{01}} \cup \mathbb{T}_{Z} \cdot z_{\varrho_{02}} \cup \mathbb{T}_{Z} \cdot z_{\varrho_{11}} \cup \mathbb{T}_{Z} \cdot z_{\varrho_{21}}\right) \subseteq X
$$

is the open subset of $X$ consisting of all points $x \in X$ having finite isotropy group $\mathbb{T}_{x}$. The projection pr: $\mathbb{Z}^{3} \rightarrow \mathbb{Z}^{2}$ defines a rational quotient $Z \rightarrow \mathbb{P}_{2}$ for the $\mathbb{T}$-action inducing a rational quotient $X \rightarrow Y$ which in turn is defined on $X_{0} \subseteq X$ and gives a surjective morphism $X_{0} \rightarrow Y$, where $Y=\mathbb{P}_{1}$.

Before entering the general case, let us give the precise definitions of the necessary concepts of quotients. For the moment, $X$ may be any variety with an action of an algebraic group $G$. As already indicated, a rational quotient for the $G$-variety $X$ is a dominant rational map $\pi: X \rightarrow Y$ such that $\pi^{*} \mathbb{K}(Y)=\mathbb{K}(X)^{G}$ holds. A representative of a rational quotient $\pi: X \rightarrow Y$ is a surjective morphism $W \rightarrow V$ representing $\pi$ on a nonempty open $G$-invariant subset $W \subseteq X$ and an open subset $V \subseteq Y$. By results of Rosenlicht, rational quotients always exist and admit a representative having $G$-orbits as its fibers [65].

Behind Construction 3.5 there is a specific rational quotient, the maximal orbit quotient. Recall that a geometric quotient of a $\mathbb{T}$-variety $X$ is a good quotient $X \rightarrow Y$ having precisely the $\mathbb{T}$-orbits as its fibers. Moreover, for any $\mathbb{T}$-variety $X$, we denote by $X_{0} \subseteq X$ the open subset consisting of all points $x \in X$ with finite isotropy group. 
DEFINITION 3.12. A maximal orbit quotient for a $\mathbb{T}$-variety $X$ is a rational quotient $\pi: X \rightarrow Y$ admitting a representative $\psi: W \rightarrow V$ and prime divisors $C_{0}, \ldots, C_{r}$ on $Y$ such that the following properties are satisfied:

(i) one has $W \subseteq X_{0}$ and the complements $X_{0} \backslash W \subseteq X_{0}$ and $Y \backslash V \subseteq Y$, both are of codimension at least two,

(ii) for every $i=0, \ldots, r$, the inverse image $\psi^{-1}\left(C_{i}\right) \subseteq W$ is a union of prime divisors $D_{i 1}, \ldots, D_{i n_{i}} \subseteq W$

(iii) all $\mathbb{T}$-invariant prime divisors of $X_{0}$ with nontrivial generic isotropy group occur among the $D_{i j}$,

(iv) every sequence $J=\left(j_{0}, \ldots, j_{r}\right)$ with $1 \leqslant j_{i} \leqslant n_{i}$ defines a geometric quotient $\psi: W_{J} \rightarrow V$ for the $\mathbb{T}$-action, where $W_{J}:=W \backslash \bigcup_{j \neq j_{i}} D_{i j}$.

We call $C_{0}, \ldots, C_{r} \subseteq Y$ a collection of doubling divisors for $\pi: X \rightarrow Y$. The closure of any $D_{i j}$ in $X$ is a $\mathbb{T}$-invariant prime divisor of $X$, again denoted by $D_{i j}$ and called a multiple divisor. Moreover, we denote by $E_{1}, \ldots, E_{m}$ the prime divisors in the complement $X \backslash X_{0}$ and call them the boundary divisors. Finally, we call $\psi: W \rightarrow V$ a big representative for $\pi: X \rightarrow Y$.

EXAMPLE 3.13. We continue Examples 3.3 and 3.11. The rational quotient $X \rightarrow Y$ arising from the projection $\mathbb{T}^{3} \rightarrow \mathbb{T}^{2}$ of tori is a maximal orbit quotient. The intersection points $c_{i}$ of $Y \subseteq \mathbb{P}_{2}$ with the coordinate axes $V\left(T_{i}\right)$ yield a collection of doubling divisors, the multiple divisors over $c_{i}$ are the $D_{i j}^{X}$ and the (only) boundary divisor is $E_{1}^{X}$.

REMARK 3.14. Observe that Definition 3.12 leaves some freedom for choosing the doubling divisors $C_{0}, \ldots, C_{r}$. Some divisors necessarily appear: the images of divisors with nontrivial finite generic isotropy group and the images of invariant divisors which cannot be separated by $\psi$. Beyond those, we are free to choose further doubling divisors $C_{i}$, which then means to insert $D_{i 1}$ accordingly.

REMARK 3.15. Let $\pi: X \rightarrow Y$ and $\pi^{\prime}: X \rightarrow Y^{\prime}$ be maximal orbit quotients for a $\mathbb{T}$-variety $X$. Then there are open subsets $U \subseteq Y$ and $U^{\prime} \subseteq Y^{\prime}$ having complements of codimension at least two and an isomorphism $U \rightarrow U^{\prime}$ which sends any collection of doubling divisors for $\pi$ to a collection of doubling divisors of $\pi^{\prime}$.

Proposition 3.16. Let $X \subseteq Z_{\Sigma}$ be an explicit $\mathbb{T}$-variety. Let $Z_{\Sigma}^{1} \subseteq Z_{\Sigma}$ be the union of $\mathbb{T}_{\Sigma}$ and all toric orbits $\mathbb{T}_{\Sigma} \cdot z_{Q_{i j}}$ and $Z_{\Delta}^{1} \subseteq Z_{\Delta}$ the union of all toric orbits 
of codimension at most one. Then, for $X_{1}:=X \cap Z_{\Sigma}^{1}$ and $Y_{1}:=Y \cap Z_{\Delta}^{1}$, we have a commutative diagram

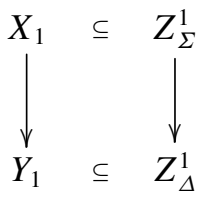

where the downwards maps are maximal orbit quotients for the action of $\mathbb{T}$. Denoting by $D_{i j}^{\Sigma}$ and $D_{k}^{\Sigma}$ the toric prime divisors of $Z_{\Sigma}$ corresponding to the rays $\varrho_{i j}=\operatorname{cone}\left(v_{i j}\right)$ and $\varrho_{k}=\operatorname{cone}\left(v_{k}\right)$, we obtain the multiple divisors and the boundary divisors of $X$ as

$$
D_{i j}^{X}=X \cap D_{i j}^{\Sigma}, \quad E_{k}^{X}=X \cap D_{k}^{\Sigma} .
$$

The generic isotropy group of $E_{k}^{X}$ is a one-dimensional torus and the generic isotropy group of $D_{i j}^{X}$ is finite of order $l_{i j}$. The doubling divisors are the intersections of $C_{i}=Y \cap D_{i}^{\Delta}$ with the toric prime divisors of $D_{i}^{\Delta} \subseteq Z_{\Delta}$.

We briefly discuss relations to polyhedral divisors [1, 2]. First we have the following recipe to convert explicit $\mathbb{T}$-varieties into the setting of polyhedral divisors.

REMARK 3.17. Given an explicit $\mathbb{T}$-variety $X \subseteq Z_{\Sigma}$, we indicate how to obtain a describing divisorial fan in the sense of [1, 2]. First follow [1, Section 11]. For every $\sigma \in \Sigma$, let $\Delta_{\sigma}$ be the fan in $\mathbb{Z}^{t}$ obtained as the coarsest common refinement of the projections $\operatorname{pr}(\tau) \subseteq \mathbb{Q}^{t}$ of all faces $\tau \preccurlyeq \sigma \subseteq \mathbb{Q}^{s+t}$. The toric variety associated with $\Delta_{\sigma}$ is the normalized Chow quotient $Z_{\sigma} / / / \mathbb{T}$; see $[26,52]$. Let $Y_{\sigma}^{\prime}$ be the normalization of the closure of the image of $X \cap \mathbb{T}_{Z}$ in $Z_{\sigma} / / / \mathbb{T}$ and write $D_{\sigma, \varrho}$ for the pull back of the toric prime divisor $D_{\varrho}$ of $Z_{\sigma} / / \mathbb{T}$ to $Y_{\sigma}^{\prime}$. Then

$$
\mathcal{D}_{\sigma}:=\sum A_{\varrho} \otimes D_{\sigma, \varrho}, \quad \mathrm{A}_{\varrho}:=\sigma \cap \operatorname{pr}^{-1}\left(v_{\varrho}\right) \subseteq \mathbb{Q}^{s+t}
$$

defines a polyhedral divisor on $Y_{\sigma}^{\prime}$ describing the $\mathbb{T}$-action on $X_{\sigma}:=X \cap Z_{\sigma}$. Now, follow the proof of [2, Theorem 5.6] to bring the local pictures together. Choose projective closures $Y_{\sigma}^{\prime} \subseteq Y_{\sigma}^{\prime \prime}$ and, via resolving indeterminacies of the birational maps between the $Y_{\sigma}^{\prime \prime}$ induced by those between the $X_{\sigma}$, construct a normal projective variety $Y^{\prime \prime}$ dominating birationally all the $Y_{\sigma}^{\prime \prime}$. Pulling back the $\mathcal{D}_{\sigma}$ to $Y^{\prime \prime}$ yields the desired divisorial fan describing the $\mathbb{T}$-action on $X$.

REMARK 3.18. In general, maximal orbit quotient and Chow quotient of a $\mathbb{T}$ variety differ from each other. For example, let $\mathbb{T}=\mathbb{K}^{*}$ act on $X=\mathbb{K}^{4}$ via

$$
t \cdot z=\left(t^{-1} z_{1}, t^{-1} z_{2}, t z_{3}, t z_{4}\right) \text {. }
$$


Working for instance in terms of fans we see that in this particular case we obtain a maximal orbit quotient just by taking the good quotient

$$
\pi: X \rightarrow X / / \mathbb{T}, \quad z \mapsto\left(z_{1} z_{3}, z_{1} z_{4}, z_{2} z_{3}, z_{2} z_{4}\right),
$$

where $X / / \mathbb{T}=\left\{w \in \mathbb{K}^{4} ; w_{1} w_{4}=w_{2} w_{3}\right\}$, and the canonical map $X / / / \mathbb{T} \rightarrow X / / \mathbb{T}$ from the Chow quotient onto the good quotient resolves the singularity $0 \in X / / \mathbb{T}$.

\section{Proofs to Section 3}

Here we prove the statements made in Construction 3.5, Proposition 3.7, Theorem 3.10 and Proposition 3.16. We will make use of Bechtold's normality criterion [12, Corollary 6]; for convenience we give a direct proof here.

Proposition 4.1. Let $K$ be a finitely generated abelian group, $R$ a $K$-factorial affine $\mathbb{K}$-algebra with only constant $K$-homogeneous units and $f_{1}, \ldots, f_{r}$ a system of pairwise nonassociated $K$-prime generators for $R$. If any $r-1$ of the $\operatorname{deg}\left(f_{i}\right)$ generate $K$ as a group, then $R$ is integral and normal.

Proof. The $K$-grading of $R$ defines an action of the quasitorus $H:=\operatorname{Spec} \mathbb{K}[K]$ on $\bar{X}:=\operatorname{Spec} R$ such that the homogeneous elements $f \in R$ of degree $w \in K$ are precisely the functions on $\bar{X}$ which are homogeneous with respect to $\chi^{w} \in \mathbb{X}(H)$. Set $g_{i}:=\prod_{j \neq i} f_{j}$ and consider the $H$-invariant open subset

$$
\hat{X}:=\bar{X}_{g_{1}} \cup \cdots \cup \bar{X}_{g_{r}} \subseteq \bar{X} .
$$

Since the $f_{i}$ are pairwise nonassociated $K$-primes, $\hat{X}$ has complement of codimension at least two in $\bar{X}$. According to [11, Theorem 1.3], each $\bar{X}_{g_{i}} / H$ is factorial and hence normal. By [5, Proposition 1.2.2.8], the $H$-action on $\bar{X}_{g_{i}}$ is free. Thus, Luna's slice theorem [55, Theorem III.1] tells us that the quotient map $\bar{X}_{g_{i}} \rightarrow \bar{X}_{g_{i}} / H$ is an étale $H$-principal bundle. As étale morphisms preserve normality, see [57, Proposition 8.1], we conclude that each $\bar{X}_{g_{i}}$ and hence $\hat{X}$ is normal. Now, observe

$$
R=\mathcal{O}(\bar{X}) \subseteq \mathcal{O}(\hat{X})
$$

We claim that the last inclusion is in fact an equality. Let $g \in \mathcal{O}(\hat{X})$ be an $H$ homogeneous function. Since $g$ is a regular homogeneous function on $\bar{X}_{g_{1}}$, we have $g=g^{\prime} / g_{1}^{l}$ with a homogeneous function $g^{\prime} \in R$. Using $K$-factoriality, we find pairwise nonassociated $K$-primes $p_{i}, f_{j} \in R$, where $f_{2}, \ldots, f_{r}$ are the generators fixed before, such that

$$
g=\frac{p_{1}^{\nu_{1}} \cdots p_{s}^{\nu_{s}}}{f_{2}^{\mu_{2}} \cdots f_{r}^{\mu_{r}}} .
$$


Since $g$ is regular on the normal variety $\hat{X}$ and $\bar{X} \backslash \hat{X}$ is of codimension at least two in $\bar{X}$, we must have $\mu_{2}=\cdots=\mu_{r}=0$. Consequently, $g \in R$ holds. Now, every regular function on $\hat{X}$ is a sum of $K$-homogeneous ones and thus extends to a regular function on $\bar{X}$. In particular, $R=\mathcal{O}(\hat{X})$ is normal.

To see that $R$ is integral, we have to show that $\bar{X}=\operatorname{Spec} R$ is irreducible. Due to normality, the irreducible components of $\bar{X}$ coincide with its connected components $\bar{X}_{1}, \ldots, \bar{X}_{k}$. Indeed, if two distinct irreducible components have a common point, then the corresponding local ring has zero divisors, contradicting normality. The assumption that $R$ is $K$-integral means on the geometric side that $H$ permutes transitively the $\bar{X}_{i}$. So, we can choose $h_{i} \in H$ with $\bar{X}_{i}=h_{i} \bar{X}_{1}$ and a nontrivial character $\chi \in \mathbb{X}(H)$ vanishing along the stabilizer of $\bar{X}_{1}$. Then, setting $f(z):=\chi\left(h_{i}\right)$ for $z \in \bar{X}_{i}$ defines a homogeneous unit on $\bar{X}$, which is nonconstant as soon as $k>1$ holds. We conclude $k=1$ and thus $\bar{X}$ is irreducible.

Proof of Construction 3.5, Propositions 3.7 and 3.16. The generator matrix $B$ of the fan $\Delta$ and the generator matrix $P$ of the fan $\Sigma$ fit into the commutative diagram

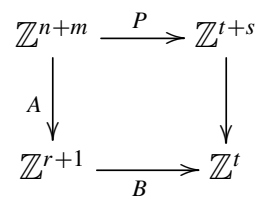

where the lifting $A: \mathbb{Z}^{n+m} \rightarrow \mathbb{Z}^{r+1}$ of the projection $\mathbb{Z}^{t+s} \rightarrow \mathbb{Z}^{t}$ sends the canonical basis vectors $e_{i j} \in \mathbb{Z}^{n+m}$ to $l_{i j} e_{i} \in \mathbb{Z}^{r+1}$ and $e_{k} \in \mathbb{Z}^{n+m}$ to $0 \in \mathbb{Z}^{r+1}$. Dualizing leads to a commutative ladder of abelian groups with exact rows

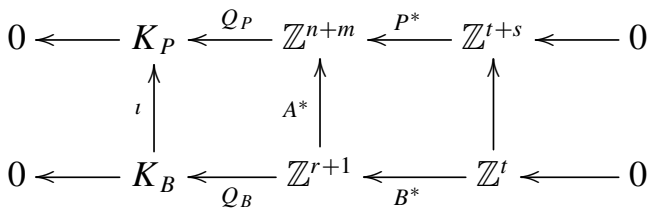

We validate Construction 3.5. According to Construction 2.8, the canonical basis vector $e_{i} \in \mathbb{Z}^{r+1}$ is sent by $Q_{B}$ to $\operatorname{deg}\left(f_{i}\right) \in K_{B}$. Thus, the induced map $\imath: K_{B} \rightarrow K_{P}$ sends $\operatorname{deg}\left(f_{i}\right) \in K_{B} Q_{P}\left(l_{i 1} e_{i 1}+\cdots+l_{i n_{i}} e_{i n_{i}}\right) \in K_{P}$. Define a $K_{B}$-grading on the polynomial ring $\mathbb{K}\left[F_{0}, \ldots, F_{r}\right]$ by $\operatorname{deg}\left(F_{i}\right)=\operatorname{deg}\left(f_{i}\right)$ and a $K_{B}$-grading on $\mathbb{K}\left[T_{i j}, S_{k}\right]$ by $\operatorname{deg}\left(T_{i j}\right)=Q_{P}\left(e_{i j}\right)$ and $\operatorname{deg}\left(S_{k}\right)=Q_{P}\left(e_{k}\right)$. Then the homomorphism

$$
\mathbb{K}\left[F_{0}, \ldots, F_{r}\right] \rightarrow \mathbb{K}\left[T_{i j}, S_{k}\right], \quad f_{i} \mapsto T_{i}^{l_{i}}
$$


sends homogeneous elements of degree $w \in K_{B}$ to homogeneous elements of degree $l(w) \in K_{P}$. In particular, the defining relations $h_{j}\left(T_{0}^{l_{0}}, \ldots, T_{r}^{l_{r}}\right)$ are $K_{P^{-}}$ homogeneous, the $K_{P}$-grading of $R(\alpha, P)$ is well defined and, moreover, we have the induced homomorphism of the graded algebras $R_{Y} \rightarrow R(\alpha, P)$ sending $f_{i}$ to $T_{i}^{l_{i}}$ as desired.

We turn to Proposition 3.7. Let $\bar{Y} \subseteq \mathbb{K}^{r+1}$ and $\bar{X} \subseteq \mathbb{K}^{n+m}$ denote the closures of the inverse images of $Y \cap \mathbb{T}^{t}$ and $X \cap \mathbb{T}^{t+s}$ under the homomorphisms of tori $b: \mathbb{T}^{r+1} \rightarrow \mathbb{T}^{t}$ and $p: \mathbb{T}^{n+m} \rightarrow \mathbb{T}^{t+s}$ defined by $B$ and $P$, respectively. Observe that $\bar{Y}=\operatorname{Spec} R_{Y}$ holds. With the quasitori $H_{Y}:=\operatorname{Spec} \mathbb{K}\left[K_{B}\right]$ and $H_{X}:=\operatorname{Spec} \mathbb{K}\left[K_{P}\right]$ and the homomorphism of tori $a: \mathbb{T}^{n+m} \rightarrow \mathbb{T}^{r+1}$ defined by $A$, we have a commutative diagram

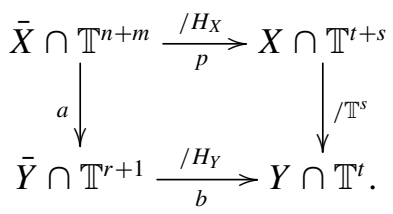

Consider the product $f \in R_{Y}$ over all the generators $f_{i}$ of $R_{Y}$ and the product $g \in R(\alpha, P)$ over all the generators $T_{i j}$ and $S_{k}$ of $R(\alpha, P)$. Then, using the above diagram, we see

$$
\left(\left(R_{Y}\right)_{f}\right)^{H_{Y}} \cong a^{*}\left(\left(R_{Y}\right)_{f}\right)^{H_{Y}}=\left(\left(R(\alpha, P)_{g}\right)^{H_{X}}\right)^{\mathbb{T}^{s}} .
$$

Since the left hand side ring is factorial, also the right hand side ring is so. By assumption, $R(\alpha, P)$ is $K_{P}$-integral and the generators $T_{i j}$ are $K_{P}$-prime. Using [11, Theorem 1.3], we see that $R(\alpha, P)$ is factorially $K_{P}$-graded and Proposition 4.1 shows that $R(\alpha, P)$ is integral and normal. Consequently, we are in the setting of Construction 2.8 which establishes Proposition 3.7.

Finally, we show Proposition 3.16. First note that $Z_{\Sigma}^{1} \rightarrow Z_{\Delta}^{1}$ defines a maximal orbit quotient of the $\mathbb{T}^{s}$-action on $Z_{\Sigma}$. The toric prime divisors of $Z_{\Sigma}^{1}$ cut down to the prime divisors $D_{i j}^{X}$ and $D_{k}^{X}$ of $X_{1}$ and those of $Z_{\Delta}^{1}$ to the prime divisors $C_{i}$ of $Y_{1}$. Thus, we can infer the statements on the isotropy groups from [5, Proposition 2.1.4.2] and conclude that $X_{1} \rightarrow Y_{1}$ is a big representative of a maximal orbit quotient of the $\mathbb{T}^{s}$-variety $X$.

We come to the proof of Theorem 3.10. The task is to provide for any abstractly given $A_{2}$-maximal $\mathbb{T}$-variety $X$ with only constant invertible global functions, finitely generated divisor class group $\mathrm{Cl}(X)$ and finitely $\mathrm{Cox}$ ring $\mathcal{R}(X)$ a presentation as an explicit $\mathbb{T}$-variety $X \subseteq Z$. This runs via general Cox ring theory. Let us recall the necessary background. Mimicking Cox's quotient 
presentation Construction 2.3, one looks at

$$
\bar{X}=\operatorname{Spec} \mathcal{R}(X), \quad H=\operatorname{Spec} \mathbb{K}[\operatorname{Cl}(X)],
$$

the total coordinate space and the characteristic quasitorus of $X$. Then $H$ acts on $\bar{X}$, where this action is defined via its comorphism, sending a homogeneous element $f \in \mathcal{R}(X)$ of degree $[D]$ to the element $\chi^{[D]} \otimes f$ of $\mathbb{K}[\mathrm{Cl}(X)] \otimes \mathcal{R}(X)$. Moreover, $X$ can be reconstructed as a good quotient

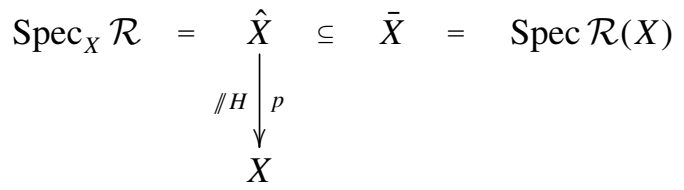

Here the relative spectrum $\hat{X}$ of the Cox sheaf $\mathcal{R}$ is called the characteristic space over $X$. It is an open $H$-invariant subset of $\bar{X}$ and the complement $\bar{X} \backslash \hat{X}$ is small in the sense that it is of codimension at least two in $\bar{X}$; see [5, Section 1.6.1].

We will deal with canonical sections, which in the context of Cox rings means the following. For any effective representative $D$ of a class $[D] \in \mathrm{Cl}(X)$, there is, up to scalars, a unique $f \in \mathcal{R}(X)_{[D]}$ with $\operatorname{div}(f)=p^{*} D$ on $\hat{X}$. In this situation, we call $f$ a canonical section of $D$ and write $f=1_{D}$. A canonical section $1_{D}$ is a $\mathrm{Cl}(X)$-prime element of $\mathcal{R}(X)$ if and only if $D$ is a prime divisor on $X$. See [5, Proposition 1.5.3.5 and Lemma 1.5.3.6] for the full details.

Proof of Theorem 3.10. Write for short $K:=\mathrm{Cl}(X)$ and $R:=\mathcal{R}(X)$. In a first step, we lift the action of the torus $\mathbb{T}$ to the total coordinate space $\bar{X}$. Consider the characteristic space $p: \hat{X} \rightarrow X$ over $X$. By [5, Theorem 4.2.3.2], there are a $\mathbb{T}$-action on $\hat{X}$ and a positive integer $b$ such that for all $t \in \mathbb{T}, h \in H$ and $x \in \hat{X}$, we have

$$
t \cdot h \cdot x=h \cdot t \cdot x, \quad p(t \cdot x)=t^{b} \cdot p(x) .
$$

Since $\hat{X} \subseteq \bar{X}$ has a small complement and $\bar{X}$ is normal, the $\mathbb{T}$-action on $\hat{X}$ extends to $\bar{X}$. The fact that the actions of $\mathbb{T}$ and $H$ commute means that we have an action of $\mathbb{T} \times H$ on $\bar{X}$. Thus, the $K$-grading of $R$ refines to a $(M \times K)$-grading for $M=\mathbb{X}(\mathbb{T})$. As $M$ is torsion free, [11, Theorem 1.5] yields that $R$ is $(M \times K)$ factorial and $(M \times K)$-primality coincides with $K$-primality in $R$.

Now, let $\mathfrak{F}=\left(f_{1}, \ldots, f_{q}\right)$ be a system of pairwise nonassociated $K$-prime generators of $R$ such that every $\mathbb{T}$-invariant prime divisor of $X$ having nontrivial generic isotropy group has a canonical section among the $f_{i}$. Then, as mentioned before, the $f_{i}$ are $(M \times K)$-prime, and thus in particular $(M \times K)$-homogeneous. Similarly as in Construction 2.8, we obtain a $(\mathbb{T} \times H)$-equivariant closed 
embedding

$$
\text { Spec } R=: \bar{X} \stackrel{x \mapsto\left(f_{1}(x), \ldots, f_{q}(x)\right)}{\longrightarrow} \bar{Z}:=\mathbb{K}^{q} .
$$

Let $Q: \mathbb{Z}^{q} \rightarrow K, e_{i} \mapsto \operatorname{deg}\left(f_{i}\right)$ be the degree map of the $K$-grading. Then, in the language of [5, Theorem 3.1.4.4], we have a maximal bunch of orbit cones

$$
\Phi=\left\{Q\left(\gamma_{x}\right) ; x \in \hat{X} \text { with } H \cdot x \subseteq \hat{X} \text { closed }\right\}, \quad \gamma_{x}=\operatorname{cone}\left(e_{i} ; f_{i}(x) \neq 0\right) .
$$

Moreover, [5, Propositions 3.2.2.2, 3.2.2.5] ensure that we obtain a bunched ring $(R, \mathfrak{F}, \Phi)$ in the sense of [5, Definition 3.2.1.1]. Now we reverse the translation performed in Remark 2.10. Fix a $q^{\prime} \times q$ matrix $P$, the rows of which form a lattice basis for $\operatorname{ker}(Q)$. For a face $\gamma_{0} \preccurlyeq \gamma$ of the orthant $\gamma=\mathbb{Q}_{\geqslant 0}^{q}$, let $\gamma_{0}^{*} \preccurlyeq \gamma$ be the complementary face. Then

$$
\Sigma_{X}:=\left\{P\left(\gamma_{x}^{*}\right) ; x \in \hat{X} \text { with } H \cdot x \subseteq \hat{X} \text { closed }\right\}
$$

is a set of cones intersecting in common faces; see [5, Theorem 2.2.1.14]. Let $\Sigma$ be any fan in $\mathbb{Z}^{q^{\prime}}$ such that $\Sigma_{X} \subseteq \Sigma$ holds. Consider the associated toric variety $Z=Z_{\Sigma}$ and Cox's quotient presentation $\hat{Z} \rightarrow Z$. We will build up the following commutative diagram

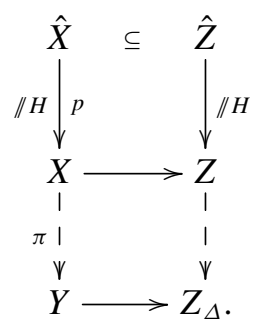

By $A_{2}$-maximality of $X$ and the choice of $\Sigma$, we have $\hat{X}=\bar{X} \cap \hat{Z}$ and the induced morphism $X \rightarrow Z$ of quotient spaces is a closed embedding. Moreover, $X \rightarrow Z$ is $\mathbb{T}$-equivariant, where $\mathbb{T}$ acts on $Z$ as a subtorus of $\mathbb{T}_{Z} \subseteq Z$. Choose a splitting $\mathbb{T}_{Z}=\mathbb{T}^{t} \times \mathbb{T}$. Accordingly, the lattice hosting the fan $\Sigma$ splits as $\mathbb{Z}^{q^{\prime}}=\mathbb{Z}^{t} \times \mathbb{Z}^{s}$. Let $\Delta$ be the fan in $\mathbb{Z}^{t}$ consisting of the zero cone and the projections of the rays of $\Sigma$. Then the projection $\mathbb{T}_{Z} \rightarrow \mathbb{T}^{t}$ of acting tori defines the rational map $Z \rightarrow Z_{\Delta}$. Defining $Y \subseteq Z_{\Delta}$ to be the closure of the image of $X \cap \mathbb{T}_{Z}$, we complete the commutative diagram.

We investigate the shape of the generator matrices $B$ of $\Delta$ and $P$ of $\Sigma$. Numbering its columns as $u_{0}, \ldots, u_{r}$, we turn $B$ into a $t \times(r+1)$ matrix. For every $i=0, \ldots, r$, denote by $v_{i 1}, \ldots, v_{i n_{i}}$ the columns of $P$ such that the ray $\varrho_{i j}=\operatorname{cone}\left(v_{i j}\right)$ projects onto cone $\left(u_{i}\right)$. Moreover, denote by $v_{1}, \ldots, v_{m}$ the columns of $P$ such that the ray $\varrho_{k}=\operatorname{cone}\left(v_{k}\right)$ lies in the kernel of the projection 
$\mathbb{Q}^{t} \times \mathbb{Q}^{s} \rightarrow \mathbb{Q}^{t}$. Then $P$ is a $(n+m) \times(t+s)$ matrix, where $n=n_{0}+\cdots+n_{r}$. Consider the toric prime divisors $D_{i j}^{Z} \subseteq Z$ and $E_{k}^{Z} \subseteq Z$ corresponding to the rays $\varrho_{i j}$ and $\varrho_{k}$, respectively. Computing the generic isotropy groups $\mathbb{T}_{x}$ of these divisors according to [5, Proposition 2.1.4.2], we see that the $E_{k}^{Z}$ are the boundary divisors of the $\mathbb{T}$-action and that the $v_{i j}$ have a nontrivial $\mathbb{Z}^{t}$-part being the $l_{i j}$ fold multiple of the primitive generator $u_{i} \in \mathbb{Z}^{t}$. Thus, $B$ and $P$ look as in Construction 3.5.

We claim that the dashed arrows are maximal orbit quotients for the $\mathbb{T}$-actions on $Z$ and $X$, respectively. Consider the union $Z^{1} \subseteq Z$ of $\mathbb{T}_{Z}$ and all toric orbits $\mathbb{T}_{Z} \cdot z_{Q_{i j}}$. Then $Z^{1} \subseteq Z_{0}$ is an open subset with complement of codimension at least two in the set $Z_{0} \subseteq Z$ consisting of all points $z \in Z$ with finite isotropy group $\mathbb{T}_{z}$. Let $C_{i}$ be the prime divisor of $Z_{\Delta}$ corresponding to cone $\left(u_{i}\right)$, where $i=0, \ldots, r$. Then $C_{0}, \ldots, C_{r}$ serve as doubling divisors and $Z^{1} \rightarrow Z_{\Delta}$ is a big representative for the rational quotient $Z \rightarrow Z_{\Delta}$, where Property 3.12(iv) is due to [5, Corollary 2.3.1.7]. Cutting down to $X$ gives an open subset $X^{1}=$ $X \cap Z^{1}$ of $X_{0}$ and a morphism $\psi: X^{1} \rightarrow Y$, which inherits the properties of a big representative from $Z^{1} \rightarrow Z_{\Delta}$. In particular, $Y$ is normal. A collection of doubling divisors is given by $C_{i}=Y \cap D_{i}$, where $D_{0}, \ldots, D_{r} \subseteq Z_{\Delta}$ are the invariant prime divisors of $Z_{\Delta}$. Observe that each $C_{i}$ is prime, because it is the image of $X \cap D$ for any $\mathbb{T}_{Z}$-invariant prime divisor $D \subseteq Z$ lying over $D_{i} \subseteq Z_{\Delta}$.

To conclude the proof, we still have to show that $Y \subseteq Z_{\Delta}$ is an explicit variety, that means that $1_{C_{0}}, \ldots, 1_{C_{r}}$ generate the Cox ring $\mathcal{R}(Y)$. Consider the commutative diagram

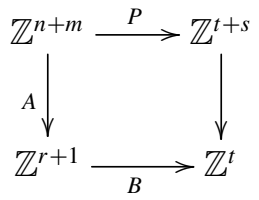

where the matrix $A: \mathbb{Z}^{n+m} \rightarrow \mathbb{Z}^{r+1}$ defines the homomorphism of $a: \mathbb{T}^{n+m} \rightarrow$ $\mathbb{T}^{r+1}$ which in turn uniquely extends to the monomial map

$$
a: \mathbb{K}^{n+m} \rightarrow \mathbb{K}^{r+1}, \quad(z, w) \mapsto\left(z_{0}^{l_{0}}, \ldots, z_{r}^{l_{r}}\right) .
$$

Note that $a$ is the good quotient for the action of the quasitorus $\operatorname{ker}(a)$ on $\mathbb{K}^{n+m}$. The total coordinate space $\bar{X} \subseteq \mathbb{K}^{n+m}$ is invariant and thus maps onto a closed normal subvariety $\bar{Y} \subseteq \mathbb{K}^{r+1}$. Moreover, $\bar{Y}$ inherits from $X$ the property that the coordinate functions of $\mathbb{K}^{r+1}$ define pairwise nonassociated elements on $\mathcal{O}(\bar{Y})$. By construction, $\bar{Y} \cap \mathbb{T}^{r+1}$ dominates $Y \subseteq Z_{\Delta}$. Thus, using [5, Lemmas 3.4.1.7, 3.4.1.9 and Corollary 3.4.1.6], we see that $\mathcal{O}(\bar{Y})$ is the Cox ring of $Y$.

As a consequence of the above proofs we retrieve [46, Theorem 1.2] for the special case of $\mathbb{T}$-varieties with finitely generated Cox ring. 
Corollary 4.2. Let $X$ be a $\mathbb{T}$-variety with finitely generated Cox ring $\mathcal{R}(X)$. Then $X$ admits a maximal orbit quotient $\pi: X \rightarrow Y$ and a collection $C_{0}, \ldots, C_{r}$ of doubling divisors such that we have an isomorphism of $\mathrm{Cl}(X)$-graded rings

$$
\mathcal{R}(X) \cong \mathcal{R}(Y)\left[T_{i j}, S_{k}\right] /\left\langle T_{i}^{l_{i}}-U_{i} ; i=0, \ldots, r\right\rangle,
$$

where $T_{i j}, S_{k} \in \mathcal{R}(X)$ and $U_{i} \in \mathcal{R}(Y)$ are canonical sections of the multiple divisors $D_{i j}$, boundary divisors $E_{k}$ and doubling divisors $C_{i}$, respectively, and the $\mathrm{Cl}(X)$-grading on the right hand side is given by

$$
\operatorname{deg}\left(U_{i}\right)=\left[l_{i 1} D_{i 1}+\cdots+l_{i n_{i}} D_{i n_{i}}\right], \quad \operatorname{deg}\left(D_{i j}\right)=\left[D_{i j}\right], \quad \operatorname{deg}\left(S_{k}\right)=\left[E_{k}\right] .
$$

Moreover, we have $\bar{Y}=\bar{X} / / H_{X, Y}$, where the quasitorus $H_{X, Y} \subseteq \mathbb{T}^{n+m}$ is the kernel of the homomorphism of tori $\mathbb{T}^{n+m} \rightarrow \mathbb{T}^{r+1}$ sending $(t, s)$ to $\left(t_{0}^{l_{0}}, \ldots, t_{r}^{l_{r}}\right)$.

\section{First properties and examples}

We discuss basic geometric properties of explicit $\mathbb{T}$-varieties. First we provide a collection of general statements directly imported from [5, Ch. 3], concerning singularities, the Picard group and various cones of divisor classes. Then we present more specific statements involving the $\mathbb{T}$-action. The second part of the section is devoted to examples. We indicate how to apply the results in practice by means of a concrete (new) example, we show how the construction of rational $\mathbb{T}$-varieties of complexity one from $[41,47]$ fits into the framework of explicit $\mathbb{T}$-varieties and finally, we present the Grassmannian $\operatorname{Gr}(2, n)$ with its maximal torus action as an explicit $\mathbb{T}$-variety.

When we speak about an explicit $\mathbb{T}$-variety $X \subseteq Z$ or, more specifically, about an explicit $\mathbb{T}$-variety $X(\alpha, P, \Sigma)$ in $Z_{\Sigma}$, then we allow ourselves to make free use of the notation introduced in Construction 3.5. Recall from Remark 3.6 that the case of a trivial $\mathbb{T}$-action, that means the explicit varieties from Construction 2.8, is included via $s=m=0$ and $n=r+1$.

REMARK 5.1. Let $X \subseteq Z$ be an explicit $\mathbb{T}$-variety. The total coordinate spaces $\bar{X}$ and $\bar{Z}$, that means the spectra of the Cox $\operatorname{rings} \mathcal{R}(X)$ and $\mathcal{R}(Z)$, are given as

$$
\bar{X}:=\bar{X}(\alpha, P):=V\left(h_{1}\left(T_{0}^{l_{0}}, \ldots, T_{r}^{l_{r}}\right), \ldots, h_{q}\left(T_{0}^{l_{0}}, \ldots, T_{r}^{l_{r}}\right)\right) \subseteq \mathbb{K}^{n+m}=: \bar{Z} .
$$

The embedding $\bar{X} \subseteq \bar{Z}$ is equivariant with respect to the actions of the characteristic quasitorus $H=\operatorname{Spec} \mathbb{K}\left[K_{P}\right]$ defined by the gradings of $\mathcal{R}(X)$ and 
$\mathcal{R}(Z)$ by $K_{P}=\mathrm{Cl}(X)=\mathrm{Cl}(Z)$. Moreover, we have a commutative diagram

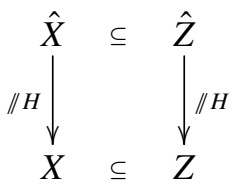

where $\hat{Z} \rightarrow Z$ is Cox's quotient presentation Construction 2.3 and $\hat{X}=\bar{X} \cap \hat{Z}$ holds. The good quotients $\hat{X} \rightarrow X$ and $\hat{Z} \rightarrow Z$ are the characteristic spaces over $X$ and $Z$, respectively.

Every explicit $\mathbb{T}$-variety $X \subseteq Z$ inherits a decomposition into locally closed subsets by cutting down the toric orbit decomposition of $Z$. Generalizing wellknown basic facts of toric geometry, one can express several geometric properties of $X$ in terms of this inherited decomposition. Let us introduce the necessary notation for precise statements.

Definition 5.2. Let $X \subseteq Z$ be an explicit $\mathbb{T}$-variety. Set $\gamma:=\mathbb{Q}_{\geqslant 0}^{n+m}$. An $\bar{X}$-face is a face $\gamma_{0} \preccurlyeq \mathbb{Q}^{n+m}$ such that the complementary face $\gamma_{0}^{*} \preccurlyeq \gamma$ satisfies

$$
\mathbb{K}^{n+m} \supseteq \bar{X}\left(\gamma_{0}\right):=\bar{X} \cap \mathbb{T}^{n+m} \cdot z_{\gamma_{0}^{*}} \neq \emptyset .
$$

For $\sigma \in \Sigma$ and the corresponding face $\gamma_{0} \preccurlyeq \gamma$, that means the face with $P\left(\gamma_{0}^{*}\right)=$ $\sigma$, consider the intersection of $X$ and the associated toric orbit of $Z=Z_{\Sigma}$ :

$$
X\left(\gamma_{0}\right):=X(\sigma):=X \cap \mathbb{T}^{t+s} \cdot z_{\sigma} \subseteq Z .
$$

We call $\sigma \in \Sigma$ an $X$-cone and $\gamma_{0} \preccurlyeq \gamma$ an $X$-face if $X\left(\gamma_{0}\right)=X(\sigma)$ is nonempty. Moreover, we denote

$$
\operatorname{rlv}(X):=\left\{\gamma_{0} \preccurlyeq \gamma ; \gamma \text { is an } X \text {-face }\right\} .
$$

Finally, we call the subsets $X\left(\gamma_{0}\right) \subseteq X$, where $\gamma_{0}$ is an $X$-face, the pieces of the explicit $\mathbb{T}$-variety $X \subseteq Z$.

REMARK 5.3. Let $X \subseteq Z$ be an explicit $\mathbb{T}$-variety. Then every piece $X\left(\gamma_{0}\right) \subseteq X$ is locally closed and $X$ is the disjoint union of its pieces:

$$
X=\bigsqcup_{\gamma_{0} \in \operatorname{rlv}(X)} X\left(\gamma_{0}\right)
$$

Moreover, $\gamma_{0} \preccurlyeq \gamma$ is an $X$-face if and only if it is an $\bar{X}$-face and we have $P\left(\gamma_{0}^{*}\right) \in$ $\Sigma$. If $\gamma_{0} \preccurlyeq \gamma$ is an $X$-face, then $\bar{X}\left(\gamma_{0}\right)$ maps onto $X\left(\gamma_{0}\right)$. 
We describe basic local properties in terms of the pieces. Consider for the moment any normal variety $X$. A point $x \in X$ is factorial if every Weil divisor of $X$ is Cartier near $x$. Moreover, $x \in X$ is $\mathbb{Q}$-factorial if for every Weil divisor of $X$ some nonzero multiple is Cartier near $x$.

Proposition 5.4. Let $X \subseteq Z$ be an explicit $\mathbb{T}$-variety. Consider an $X$-face $\gamma_{0} \preccurlyeq$ $\gamma$ and $\sigma=P\left(\gamma_{0}^{*}\right) \in \Sigma$. Then the following statements are equivalent.

(i) The piece $X(\sigma)$ consists of $\mathbb{Q}$-factorial points of $X$.

(ii) The cone $\sigma$ is simplicial.

(iii) The cone $Q\left(\gamma_{0}\right) \subseteq K_{\mathbb{Q}}$ is of full dimension.

Proof. Translate via Remark 2.10 and apply [5, 3.3.1.8 and 3.3.1.12].

Proposition 5.5. Let $X \subseteq Z$ be an explicit $\mathbb{T}$-variety. Consider an $X$-face $\gamma_{0} \preccurlyeq$ $\gamma$ and $\sigma=P\left(\gamma_{0}^{*}\right) \in \Sigma$. Then the following statements are equivalent.

(i) The piece $X(\sigma)$ consists of factorial points of $X$.

(ii) The cone $\sigma$ is regular.

(iii) The set $Q\left(\gamma_{0} \cap \mathbb{Z}^{n+m}\right)$ generates $K$ as a group.

Moreover, $X(\sigma)$ consists of smooth points of $X$ if and only if one of the above statements holds and $\bar{X}\left(\gamma_{0}\right)$ consists of smooth points of $\bar{X}$.

Proof. Translate via Remark 2.10 and apply [5, 3.3.1.8, 3.3.1.9 and 3.3.1.12].

We turn to the Picard group and the various cones of divisor classes. For a subset $A \subseteq V$ of a $\mathbb{Q}$-vector space, we denote by $\operatorname{lin}(A) \subseteq V$ its $\mathbb{Q}$-linear hull.

Proposition 5.6. Let $X \subseteq Z$ be an explicit $\mathbb{T}$-variety. Then, in $K_{P}=\mathrm{Cl}(X)$, the Picard group of $X$ is given by

$$
\operatorname{Pic}(X)=\bigcap_{\gamma_{0} \in \operatorname{rlv}(X)} Q\left(\operatorname{lin}\left(\gamma_{0}\right) \cap \mathbb{Z}^{n+m}\right) .
$$

Moreover, in $\left(K_{P}\right)_{\mathbb{Q}}=\mathrm{Cl}_{\mathbb{Q}}(X)$, the cones of effective, movable, semiample and ample divisor classes are given by

$$
\operatorname{Eff}(X)=Q(\gamma), \quad \operatorname{Mov}(X)=\bigcap_{\gamma_{0} \preccurlyeq \gamma \text { facet }} Q\left(\gamma_{0}\right),
$$




$$
\operatorname{SAmple}(X)=\bigcap_{\gamma_{0} \in \operatorname{rlv}(X)} Q\left(\gamma_{0}\right), \quad \operatorname{Ample}(X)=\bigcap_{\gamma_{0} \in \operatorname{rlv}(X)} Q\left(\gamma_{0}\right)^{\circ}
$$

Proof. Translate via Remark 2.10 and apply [5, Corollary 3.3.1.6 and Proposition 3.3.2.9].

REMARK 5.7. Let $X=X(\alpha, P, \Sigma)$ in $Z=Z_{\Sigma}$ be an explicit $\mathbb{T}$-variety. If the fan $\Sigma$ is the normal fan of a polytope in $\mathbb{Q}^{t+s}$, then $Z$ and hence $X$ are projective. Conversely, if $X$ is projective, choose any class $u \in \operatorname{Ample}(X)$, an element $e \in$ $\mathbb{Q}^{n+m}$ with $Q(e)=u$ and consider the polytope

$$
\left.B(u)=\left(P^{*}\right)^{-1}\left(Q^{-1}(u) \cap \gamma\right)-e\right) \subseteq \mathbb{Q}^{t+s} .
$$

Then, with the normal fan $\Sigma(u)$ of $B(u)$, we have $X=X(\alpha, P, \Sigma(u))$, whereas the toric ambient variety $Z(u)$ associated with $\Sigma(u)$ may differ from the original $Z=Z_{\Sigma}$. Note that in terms of the faces $\gamma_{0} \preccurlyeq \gamma$, the normal fan is given as

$$
\Sigma(u)=\left\{P\left(\gamma_{0}^{*}\right) ; \gamma_{0} \preccurlyeq \gamma \text { with } u \in Q\left(\gamma_{0}\right)^{\circ}\right\} .
$$

We indicate, in our setting, the fundamental connection between geometric invariant theory and Mori theory found by $\mathrm{Hu}$ and Keel [48, Theorem 2.3]; we refer to [5, Sections 3.1.2 and 3.3.4] for additional background.

REMARK 5.8. Let $X=X(\alpha, P, \Sigma(u))$ in $Z=Z_{\Sigma(u)}$ be a projective explicit $\mathbb{T}$ variety. For every $u \in \operatorname{Eff}(X)$, denote by $\Gamma(u)$ the collection of $\bar{X}$-faces $\gamma_{0} \preceq \gamma$ with $u \in Q\left(\gamma_{0}\right)$ and define a convex polyhedral cone

$$
\lambda_{u}=\bigcap_{\gamma_{0} \in \Gamma(u)} Q\left(\gamma_{0}\right) \subseteq K_{\mathbb{Q}}=\mathrm{Cl}_{\mathbb{Q}}(X)
$$

The cones $\lambda_{u}$ form a fan $\Lambda$ subdividing $\operatorname{Eff}(X)$. The fan $\Lambda$ maintains the geometric invariant theory of the characteristic quasitorus action on $\bar{X}$ in the sense that the sets $X^{s s}(u)$ of semistable points associated with the characters $\chi^{u} \in \mathbb{X}(H)$ satisfy

$$
\bar{X}^{s s}(u) \subseteq \bar{X}^{s s}\left(u^{\prime}\right) \quad \Longleftrightarrow \quad \lambda_{u} \succcurlyeq \lambda_{u^{\prime}} .
$$

Observe that if one of the conditions holds, then we have an induced morphism $X_{u} \rightarrow X_{u^{\prime}}$ of the associated quotients by $H$. Now, look at the $u \in \operatorname{Mov}(X)^{\circ}$. These define projective explicit $\mathbb{T}$-varieties. More precisely, we have

$$
X_{u}=X(\alpha, P, \Sigma(u)), \quad \hat{X}_{u}=\bar{X}^{s s}(u) .
$$

Each $X_{u}$ has $\lambda_{u}$ as its semiample cone and is $\mathbb{Q}$-factorial if and only if $\operatorname{dim}\left(\lambda_{u}\right)$ equals $\operatorname{dim}\left(K_{\mathbb{Q}}\right)$. Moreover, $\Lambda$ reflects the Mori equivalence: the birational map $X_{u} \rightarrow X_{u}^{\prime}$ is an isomorphism if and only if $u, u^{\prime} \in \lambda^{\circ}$ holds for some $\lambda \in \Lambda$. 
Now we discuss more specific properties of explicit $\mathbb{T}$-varieties $X \subseteq Z$ involving in particular the torus action. Recall that $\mathbb{T}=\mathbb{T}^{s}$, being a factor of $\mathbb{T}_{Z}=\mathbb{T}^{t} \times \mathbb{T}^{s}$, acts on $Z$ and leaves $X \subseteq Z$ invariant. Moreover, the projection $\mathbb{T}_{Z} \rightarrow \mathbb{T}^{t}$ defines the maximal orbit quotient $Z \rightarrow Z_{\Delta}$ for the $\mathbb{T}$-action on $Z$ and by restricting we obtain a maximal orbit quotient $\pi: X \rightarrow Y$ for the $\mathbb{T}$-action on $X$.

Proposition 5.9. Let $X \subseteq Z$ be an explicit $\mathbb{T}$-variety. Let $L \subseteq \mathbb{Z}^{t+s}$ be the kernel of the projection $\mathrm{pr}: \mathbb{Z}^{t+s} \rightarrow \mathbb{Z}^{t}$. Then, for every $X$-cone $\sigma \in \Sigma$ and every $x \in X(\sigma)$, the isotropy group $\mathbb{T}_{x}$ satisfies

$$
\mathbb{X}\left(\mathbb{T}_{x}\right) \cong(L \cap \operatorname{lin}(\sigma)) \oplus\left(\operatorname{pr}(\operatorname{lin}(\sigma)) \cap \mathbb{Z}^{t}\right) /\left(\operatorname{pr}\left(\operatorname{lin}(\sigma) \cap \mathbb{Z}^{t+s}\right) .\right.
$$

Proof. From [5, Proposition 2.1.4.2] we infer the formula for the isotropy group of $\mathbb{T} \subseteq \mathbb{T}_{Z}$ at the point $z_{\sigma} \in Z$. Since the isotropy groups of the $\mathbb{T}$-action are constant along the toric orbits, this is all we need.

Proposition 5.10. Let $X \subseteq Z$ be an explicit $\mathbb{T}$-variety. Suppose that the Cox ring presentation $\mathcal{R}(Y)=\mathbb{K}\left[f_{1}, \ldots, f_{r}\right] /\left\langle h_{1}, \ldots, h_{q}\right\rangle$ is a complete intersection. Then, with $h_{u}^{\prime}:=h_{u}\left(T_{0}^{l_{0}}, \ldots, T_{r}^{l_{r}}\right)$, also the Cox ring presentation

$$
\mathcal{R}(X)=\mathbb{K}\left[T_{i j}, S_{k}\right] /\left\langle h_{1}^{\prime}, \ldots, h_{q}^{\prime}\right\rangle
$$

is a complete intersection. Moreover, in the latter case, the canonical divisor class of $X$ is given by

$$
\mathcal{K}_{X}=-\sum_{i=0}^{r} \sum_{j=1}^{n_{i}} \operatorname{deg}\left(T_{i j}\right)-\sum_{k=1}^{m} \operatorname{deg}\left(S_{k}\right)+\sum_{u=1}^{q} \operatorname{deg}\left(h_{u}^{\prime}\right) \in K_{P}=\operatorname{Cl}(X) .
$$

In particular, with the canonical divisor class $\mathcal{K}_{Y} \in K_{B}=\mathrm{Cl}(Y)$ and the maximal orbit quotient $\pi: X \rightarrow Y$, we have

$$
\mathcal{K}_{X}-\pi^{*}\left(\mathcal{K}_{Y}\right)=\sum_{i=0}^{r} \sum_{j=1}^{n_{i}}\left(l_{i j}-1\right) \operatorname{deg}\left(T_{i j}\right)-\sum_{k=1}^{m} \operatorname{deg}\left(S_{k}\right) .
$$

Proof. The second and third statements follow from [5, Proposition 3.3.3.2]. The first one is seen via a simple dimension computation:

$$
\begin{aligned}
\operatorname{dim}(\bar{X}) & =\operatorname{dim}(X)+\operatorname{rk}(\operatorname{Cl}(X)) \\
& =s+\operatorname{dim}(Y)+\operatorname{rk}(\operatorname{Cl}(X)) \\
& =s+\operatorname{dim}(\bar{Y})-\operatorname{rk}(\operatorname{Cl}(Y))+\operatorname{rk}(\mathrm{Cl}(X)) \\
& =s+(r+1-q)-(r+1-t)+(n+m-t-s) \\
& =n+m-q .
\end{aligned}
$$


For the next observation, note that in Construction 3.5, we may remove successively all maximal cones from the fan $\Sigma$ that are not $X$-cones. The result is a minimal fan $\Sigma$ defining still the initial $X$. We call $Z=Z_{\Sigma}$ in this case the minimal ambient toric variety of $X$.

Proposition 5.11. Let $X \subseteq Z$ be an explicit $\mathbb{T}$-variety and assume that $Z$ is the minimal toric ambient variety of $X$. Let $L \subseteq \mathbb{Z}^{t+s}$ be the kernel of the projection $\mathbb{Z}^{t+s} \rightarrow \mathbb{Z}^{t}$

(i) The normalization of the general $\mathbb{T}$-orbit closure of $X$ is the toric variety defined by the fan $\Sigma_{L}$ in $L$, where

$$
\Sigma_{L}:=\left\{\tau ; \tau \preccurlyeq\left(\sigma \cap L_{\mathbb{Q}}\right), \sigma \in \Sigma\right\} .
$$

(ii) If the maximal orbit quotient $\pi: X \rightarrow Y$ is a morphism, then $\Sigma_{L}$ is a subfan of $\Sigma$.

Proof. As $Z$ is the minimal toric embedding, the general $\mathbb{T}$-orbit closure of $X$ equals the general $\mathbb{T}$-orbit closure of $Z$. This reduces the problem to standard toric geometry.

Corollary 5.12. Assumptions as in Proposition 5.11. If $X$ is complete and $\Sigma_{L}$ is a subfan of $\Sigma$, then we have

$$
\text { rk }(\mathrm{Cl}(X))-\operatorname{rk}(\mathrm{Cl}(Y))>n-r-1 .
$$

Proof. According to Proposition 5.11, the general $\mathbb{T}$-orbit closure of $X$ has divisor class group of rank $m-s>0$. Thus, the assertion follows from

$$
\operatorname{rk}(\mathrm{Cl}(X))=n+m-t-s, \quad \operatorname{rk}(\mathrm{Cl}(Y))=r+1-t .
$$

We come to the announced example discussions. First, we use Construction 3.5 to produce a concrete example of a $\mathbb{Q}$-factorial Fano variety with torus action of complexity two and maximal orbit quotient $X \rightarrow \mathbb{P}_{1} \times \mathbb{P}_{1}$.

EXAmple 5.13. Consider the surface $Y:=\mathbb{P}_{1} \times \mathbb{P}_{1}$. Then we have $\mathrm{Cl}(Y)=\mathbb{Z}^{2}$ and the Cox ring of $Y$ is the polynomial ring $\mathbb{K}\left[T_{0}, T_{1}, T_{2}, T_{3}\right]$, where the $\mathbb{Z}^{2}$ grading is given by

$$
\operatorname{deg}\left(T_{0}\right)=\operatorname{deg}\left(T_{1}\right)=(1,0), \quad \operatorname{deg}\left(T_{2}\right)=\operatorname{deg}\left(T_{3}\right)=(0,1) .
$$

Consider the redundant system $\alpha=\left(f_{0}, \ldots, f_{5}\right)$ of generators for $\mathcal{R}(Y)$ consisting of $f_{i}:=T_{i}$ for $i=0, \ldots, 3$ and the defining equations of the 
diagonals

$$
f_{4}:=T_{0} T_{3}-T_{1} T_{2}, \quad f_{5}:=T_{0} T_{2}-T_{1} T_{3},
$$

both being of degree $(1,1)$. A matrix $B$ of relations between the degrees of generators $f_{0}, \ldots, f_{5}$ is given by

$$
B:=\left[\begin{array}{rrrrrr}
-1 & 1 & 0 & 0 & 0 & 0 \\
0 & 0 & -1 & 1 & 0 & 0 \\
-1 & 0 & -1 & 0 & 1 & 0 \\
-1 & 0 & -1 & 0 & 0 & 1
\end{array}\right] .
$$

Then $Y$ is embedded into the toric variety $Z_{\Delta}$, the fan $\Delta$ of which lives in $\mathbb{Z}^{4}$ and has the following four cones as its maximal ones

$$
\text { cone }\left(v_{1}, v_{3}, v_{4}\right) \text {, cone }\left(v_{1}, v_{2}, v_{5}\right) \text {, cone }\left(v_{0}, v_{3}, v_{5}\right) \text {, cone }\left(v_{0}, v_{2}, v_{4}\right) \text {, }
$$

where $v_{i}$ denotes the $i$ th column of $B$. Note that $Y$ is given in Cox coordinates by the equation $f_{4}=f_{0} f_{3}-f_{1} f_{2}$ and $f_{5}=f_{0} f_{2}-f_{1} f_{3}$. To build the variety $X$, consider the matrix

$$
P:=\left[\begin{array}{rrrrrrrr}
-1 & 1 & 0 & 0 & 0 & 0 & 0 & 0 \\
0 & 0 & -1 & 1 & 0 & 0 & 0 & 0 \\
-1 & 0 & -1 & 0 & 2 & 0 & 0 & 0 \\
-1 & 0 & -1 & 0 & 0 & 1 & 2 & 0 \\
-1 & -1 & 1 & 1 & -1 & 1 & -1 & -1 \\
0 & 1 & 0 & 1 & 0 & 1 & 2 & -1
\end{array}\right]
$$

obtained from $B$ by first doubling the last column, then multiplying its last and third last columns with 2 , adding a zero column and, after that, adding two new rows as $d, d^{\prime}$ part. We gain polynomials by modifying the variables of the describing relations of $Y \subseteq Z_{\Delta}$ accordingly to the column modifications:

$$
g_{1}:=T_{41}^{2}-T_{01} T_{31}+T_{11} T_{21}, \quad g_{2}:=T_{51} T_{52}^{2}-T_{01} T_{21}+T_{11} T_{31} .
$$

By construction, the polynomials $g_{i}$ are homogeneous with respect to the grading of $\mathbb{K}\left[T_{i j}, S_{1}\right]$ given by

$$
\operatorname{deg}\left(T_{i j}\right):=Q\left(e_{i j}\right) \in K, \quad \operatorname{deg}\left(S_{1}\right):=Q\left(e_{1}\right) \in K,
$$

where $Q: \mathbb{Z}^{8} \rightarrow K:=\mathbb{Z}^{8} / \operatorname{im}\left(P^{*}\right) \cong \mathbb{Z}^{2}$, is the projection and $e_{i j}, e_{1} \in \mathbb{Z}^{8}$ are the canonical basis vectors, numbered according to the variables $T_{i j}$ and $S_{1}$. Let $\Sigma=\Sigma(u)$ in $\mathbb{Z}^{6}$ be the normal fan of the polytope

$$
\left.\left(P^{*}\right)^{-1}\left(Q^{-1}(u) \cap \gamma\right)-e\right) \subseteq \mathbb{Q}^{6},
$$


where $u:=(8,-4) \in K$ and $e \in \mathbb{Z}^{8}$ is any point with $Q(e)=u$. Then $\Sigma$ has the columns of $P$ as its primitive generators. Moreover, the projection $\mathbb{Z}^{8} \rightarrow \mathbb{Z}^{6}$ onto the first six coordinates sends the rays of $\Sigma$ into the rays of $\Delta$. This gives a rational toric map $\pi: Z_{\Sigma} \rightarrow Z_{\Delta}$. Now, define a variety

$$
X=X(\alpha, P, \Sigma):=\overline{\pi^{-1}\left(Y \cap \mathbb{T}^{4}\right)} \subseteq Z_{\Sigma} .
$$

Then $X$ is invariant under the action of the subtorus $\mathbb{T}:=\left\{\mathbb{1}_{4}\right\} \times \mathbb{T}^{2}$ of the acting torus $\mathbb{T}^{6}$ of $Z$. The $\mathbb{T}$-variety $X$ is normal, of dimension four with divisor class group and Cox ring given by

$$
\mathrm{Cl}(X)=\mathbb{Z}^{2}, \quad \mathcal{R}(X)=\mathbb{K}\left[T_{i j}, S_{1}\right] /\left\langle g_{1}, g_{2}\right\rangle,
$$

where the grading of the Cox ring is the one given above. This involves application of Proposition 3.7; the necessary assumptions are directly verified. Now, applying Propositions 5.4, 5.6, 5.10 and their implementation in [43], we see that $X$ is a $\mathbb{Q}$-factorial Fano variety of Gorenstein index 30 .

REMARK 5.14. The Cox ring based approach of [41, 47] produces all $A_{2}$ maximal rational $\mathbb{T}$-varieties $X$ of complexity one with only constant invertible global functions via a construction having a pair of matrices and a bunch of cones as input data. Let us see how to retrieve these $X$ via Construction 3.5. Two types of $\mathbb{T}$-varieties are distinguished: the first admits nonconstant $\mathbb{T}$-invariant functions, the second does not.

Type 1 . The starting variety is $Y=\mathbb{K}$ in $Z_{\Delta}=\mathbb{K}^{r+1}$, where the generator matrix of $\Delta$ is $B=\mathbb{E}_{r+1}$, we embed via the system $\alpha=\left(f_{0}, \ldots, f_{r}\right)$ given by $f_{i}=T-a_{i}$ with pairwise different $a_{i} \in \mathbb{K}$ and the defining relations for $Y$ are

$$
h_{i}=U_{i}-U_{i+1}-\left(a_{i}+a_{i+1}\right) \in \mathbb{K}\left[U_{0}, \ldots, U_{r}\right], \quad i=0, \ldots, r-1 .
$$

Type 2. The starting variety is $Y=\mathbb{P}_{1}$ in $Z_{\Delta}=\mathbb{P}_{r}$, where $\Delta$ has generator matrix $B=\left[-\mathbb{1}_{r}, \mathbb{E}_{r}\right]$, the embedding system $\alpha=\left(f_{0}, \ldots, f_{r}\right)$ with $f_{i}:=a_{i, 1} T_{1}+a_{i, 2} T_{2}$ such that $\left[a_{i, 1}, a_{i, 2}\right] \in \mathbb{P}_{1}$ are pairwise different and the defining relations for $Y$ are

$$
h_{i}:=\operatorname{det}\left[\begin{array}{ccc}
a_{i, 1} & a_{i+1,1} & a_{i+2,1} \\
a_{i, 2} & a_{i+1,2} & a_{i+2,2} \\
U_{i} & U_{i+1} & U_{i+2}
\end{array}\right] \in \mathbb{K}\left[U_{0}, \ldots, U_{r}\right], \quad i=0, \ldots, r-1 .
$$

Now run Construction 3.5 for both types. The assumptions of Proposition 3.7 are satisfied by [41, Theorem 10.4] and [47, Theorem 1.5]. Thus, Theorem 3.10 gives the desired result. The input data $A, P$ and $\Phi$ of $[41,47]$ are recovered as follows: 
the matrix $A$ is $\left[a_{0}, \ldots, a_{r}\right]$, where for Type 1 the $a_{i}$ are as above and for Type 2 we set $a_{i}=\left(a_{i, 1}, a_{i, 2}\right)$, the matrix $P$ is the one produced by Construction 3.5 and the bunch of cones $\Phi$ is related to the fan $\Sigma$ via Gale duality as outlined in Remark 2.10.

EXAMPle 5.15. Fix $n \geqslant 5$ and let $X_{2, n}=\operatorname{Gr}(2, n)$ be the Grassmannian of two-dimensional vector subspaces of $\mathbb{K}^{n}$. Then $X_{2, n}$ has the projective linear group PGL $(n)$ as its automorphism group [22]. We will pick a maximal torus $\mathbb{T} \subseteq \operatorname{PGL}(n)$ and show how to obtain the $\mathbb{T}$-variety $X_{2, n}$ via Construction 3.5. Set

$$
r:=\left(\begin{array}{l}
n \\
2
\end{array}\right)
$$

Identify the Plücker coordinate space $\mathbb{K}^{n} \wedge \mathbb{K}^{n}$ with $\mathbb{K}^{r}$ such that the basis $\left(e_{i} \wedge e_{j}\right)$ of $\mathbb{K}^{n} \wedge \mathbb{K}^{n}$ corresponds to the basis $\left(e_{i j}\right)$ of $\mathbb{K}^{r}$, where $e_{i j} \in \mathbb{K}^{r}$ has $i j$ th Plücker coordinate equal to one and all others zero. We order the bases $\left(e_{i} \wedge e_{j}\right)$ and $\left(e_{i j}\right)$ lexicographically. Accordingly, we have the Plücker ideal and the affine cone

$$
I_{2, n} \subseteq \mathbb{K}\left[T_{i j} ; 1 \leqslant i<j \leqslant n\right], \quad \bar{X}_{2, n}=V\left(I_{2, n}\right) \subseteq \mathbb{K}^{r} .
$$

Look at the largest diagonal torus $\overline{\mathbb{T}} \subseteq \mathrm{GL}(r)$ leaving $\bar{X}_{2, n}$ invariant. With $t:=$ $r-n$, we obtain $\overline{\mathbb{T}}$ as the kernel of the homomorphism $b: \mathbb{T}^{r} \rightarrow \mathbb{T}^{t}$ defined by the following $t \times r$ matrix, the first $n$ columns of which are defined by the remaining ones as indicated:

$$
B=\left[v_{12}, \ldots, v_{n-1 n}\right]=\left[v_{12}, \ldots, v_{1 n}, \mathbb{E}_{t},-\mathbb{1}_{t}\right], \quad v_{1 i}:=\sum_{\{j, k\} \cap\{1, i\}=\emptyset} v_{j k}
$$

Observe that $\overline{\mathbb{T}} \subseteq \mathrm{GL}(r)$ is of dimension $n$. The corresponding torus $\mathbb{T} \in \operatorname{PGL}(r)$ is of dimension $n-1$. Moreover, $\mathbb{T}$ acts effectively on $X_{2, n} \subseteq \mathbb{P}_{r-1}$ and thus $\mathbb{T}$ defines a maximal torus of the automorphism group PGL $(n)$. Now, look at the $(r-1) \times r$ stack matrix

$$
P:=\left[\begin{array}{l}
B \\
d
\end{array}\right]=\left[\begin{array}{ccc}
v_{12}, \ldots, v_{1 n} & \mathbb{E}_{t}-\mathbb{1}_{t} \\
\mathbb{E}_{n-1} & 0-\mathbb{1}_{n-1}
\end{array}\right]
$$

Observe that the kernel of $P$ is generated by the vector $\mathbb{1}_{r} \in \mathbb{Z}^{r}$. In particular, $P$ differs from $\left[\mathbb{E}_{r},-\mathbb{1}_{r}\right]$ by multiplication with a unimodular matrix from the left. Let $\Sigma$ be the unique complete fan in $\mathbb{Z}^{r}$ having $P$ as generator matrix and let $\Delta$ be the fan in $\mathbb{Z}^{t}$ having the rays through the columns of $B$ as its maximal cones. 
Then we obtain a commutative diagram

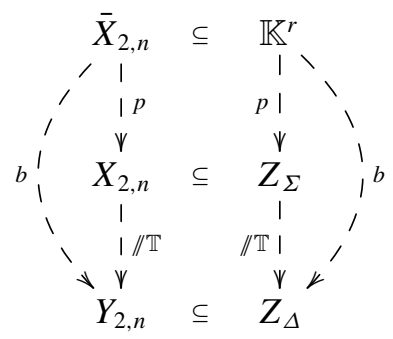

where $Y_{2, n} \subseteq Z_{\Delta}$ is the closure of the image $b\left(\bar{X}_{2, n} \cap \mathbb{T}^{r}\right)$. We have $Z_{\Sigma}=\mathbb{P}_{r-1}$ and $X_{2, n} \subseteq Z_{\Sigma}$ is the Plücker embedding. Moreover, $R_{2, n}=\mathbb{K}\left[T_{i j}\right] / I_{2, n}$ is a unique factorization domain [66, Proposition 8.5] and the variables $T_{i j}$ define prime elements. Thus, $Y_{2, n} \subseteq Z_{\Delta}$ is an explicit variety and the $\mathbb{T}$-variety $X_{2, n} \subseteq Z_{\Sigma}$ is an explicit $\mathbb{T}$-variety. Moreover, the $\mathbb{T}$-action has maximal orbit quotient

$$
\pi: X_{2, n} \rightarrow Y_{2, n} \text {. }
$$

We claim that, up to codimension two, $Y_{2, n}$ equals the blowing up $\mathrm{Bl}_{n-1}\left(\mathbb{P}_{n-3}\right)$ of $\mathbb{P}_{n-3}$ at $n-1$ points in general position. Indeed, we may first blow up the $n-2$ toric fixed points of $\mathbb{P}_{n-3}$ and then the base point of the resulting toric variety. Doing the latter via [44, Algorithm 5.7], one directly checks that the procedure terminates after the first step and delivers $R_{2, n}$ as Cox ring of $\mathrm{Bl}_{n-1}\left(\mathbb{P}_{n-3}\right)$. Thus, we may also take $X_{2, n} \rightarrow \mathrm{Bl}_{n-1}\left(\mathbb{P}_{n-3}\right)$ as a maximal orbit quotient for the $\mathbb{T}$-action.

REMARK 5.16. In order to describe a Mori dream space with torus action via divisorial fans $[1,2]$, it happens that one has to start with a non Mori dream space as prospective Chow quotient. For example, the maximal torus action on the Grassmannian $\operatorname{Gr}(2, n)$ has the moduli space $\bar{M}_{0, n}$ as its Chow quotient [51] and for $n \geqslant 10$, it is known that $\bar{M}_{0, n}$ and hence all its blow ups have a nonfinitely generated Cox ring $[21,36,45]$. Note that the Chow quotient $\bar{M}_{0, n}$ starts differing at $n=6$ from the maximal orbit quotient discussed just before. Altmann and Hein gave in [3] a description of the maximal torus action on $\operatorname{Gr}(2, n)$ by means of a divisorial fan living on $\bar{M}_{0, n}$.

\section{General arrangement varieties}

We introduce general arrangement varieties as certain $\mathbb{T}$-varieties with a projective space as maximal orbit quotient, naturally generalizing the rational projective $\mathbb{T}$-varieties of complexity one. Construction 6.13 produces examples 
which are explicit in the sense of Construction 3.5 and Theorem 6.18 shows that we obtain all $A_{2}$-maximal general arrangement varieties in this way.

For the precise definition, let us recall the necessary notions on projective hyperplane arrangements. A hyperplane $H$ in the projective space $\mathbb{P}_{n}$ is the zero set of a nonzero homogeneous polynomial of degree one. A hyperplane arrangement in $\mathbb{P}_{n}$ is a finite collection $H_{1}, \ldots, H_{r}$ of hyperplanes in $\mathbb{P}_{n}$. A hyperplane arrangement in $\mathbb{P}_{n}$ is called general if for any $1 \leqslant i_{1}<\cdots<i_{k} \leqslant r$, the intersection $H_{i_{1}} \cap \cdots \cap H_{i_{k}}$ is of dimension $(n-k)$.

Definition 6.1. A (general) arrangement variety of complexity $c$ is a $\mathbb{T}$ variety $X$ with maximal orbit quotient $X \rightarrow \mathbb{P}_{c}$ such that the doubling divisors $C_{0}, \ldots, C_{r}$ form a (general) hyperplane arrangement in $\mathbb{P}_{c}$.

REMARK 6.2. The projective general arrangement varieties of complexity $c=1$ are precisely the rational projective $\mathbb{T}$-varieties of complexity one. Indeed, any rational projective $\mathbb{T}$-variety $X$ of complexity one has maximal orbit quotient $\pi: X \rightarrow \mathbb{P}_{1}$. The doubling divisors form a point configuration in $\mathbb{P}_{1}$, which trivially satisfies the conditions of a general hyperplane arrangement.

We enter the construction of general arrangement varieties. As in the case of complexity one $[\mathbf{5}, \mathbf{4 1}, \mathbf{4 2}]$, we first write down the prospective Cox rings in terms of generators and relations, then investigate their algebraic properties and after all that construct the varieties we are aiming for.

Construction 6.3. Fix integers $r \geqslant c>0$ and $n_{0}, \ldots, n_{r}>0$ as well as $m \geqslant 0$. Set $n:=n_{0}+\cdots+n_{r}$. The input data is a pair $\left(A, P_{0}\right)$, where

- $A$ is a $(c+1) \times(r+1)$ matrix over $\mathbb{K}$ such that any $c+1$ of its columns $a_{0}, \ldots, a_{r}$ are linearly independent,

- $P_{0}$ is an integral $r \times(n+m)$ matrix built from tuples of positive integers $l_{i}=\left(l_{i 1}, \ldots, l_{i n_{i}}\right)$, where $i=0, \ldots, r$, as follows

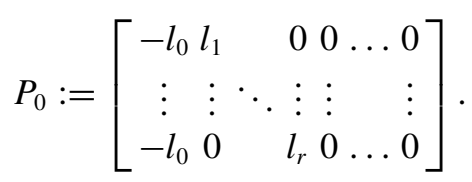

Write $\mathbb{K}\left[T_{i j}, S_{k}\right]$ for the polynomial ring in the variables $T_{i j}$, where $i=0, \ldots, r$, $j=1, \ldots, n_{i}$, and $S_{k}$, where $k=1, \ldots, m$. Every $l_{i}$ defines a monomial

$$
T_{i}^{l_{i}}:=T_{i 1}^{l_{11}} \cdots T_{i n_{i}}^{l_{i n_{i}}} \in \mathbb{K}\left[T_{i j}, S_{k}\right]
$$


Moreover, for every $t=1, \ldots, r-c$, we obtain a polynomial $g_{t}$ by computing the following $(c+2) \times(c+2)$ determinant

$$
g_{t}:=\operatorname{det}\left[\begin{array}{cccc}
a_{0} & \ldots & a_{c} & a_{c+t} \\
T_{0}^{l_{0}} & \ldots & T_{c}^{l_{c}} & T_{c+t}^{l_{c+t}}
\end{array}\right] \in \mathbb{K}\left[T_{i j}, S_{k}\right]
$$

Now, let $e_{i j} \in \mathbb{Z}^{n}$ and $e_{k} \in \mathbb{Z}^{m}$ denote the canonical basis vectors and consider the projection

$$
Q_{0}: \mathbb{Z}^{n+m} \rightarrow K_{0}:=\mathbb{Z}^{n+m} / \operatorname{im}\left(P_{0}^{*}\right)
$$

onto the factor group by the row lattice of $P_{0}$. Then the $K_{0}$-graded $\mathbb{K}$-algebra associated with $\left(A, P_{0}\right)$ is defined by

$$
\begin{gathered}
R\left(A, P_{0}\right):=\mathbb{K}\left[T_{i j}, S_{k}\right] /\left\langle g_{1}, \ldots, g_{r-c}\right\rangle, \\
\operatorname{deg}\left(T_{i j}\right):=Q_{0}\left(e_{i j}\right), \quad \operatorname{deg}\left(S_{k}\right):=Q_{0}\left(e_{k}\right) .
\end{gathered}
$$

EXAMPLE 6.4. Let us take $c=2$ and $r=3$. Thus, we will work with a $3 \times 4$ matrix $A$. Moreover, let $n_{0}=2$ and $n_{1}=n_{2}=n_{3}=1$ and fix $m=0$. This amounts to $n=5$ and a $3 \times 5$ matrix $P_{0}$. We choose

$$
A=\left[\begin{array}{llll}
1 & 0 & 0 & -1 \\
0 & 1 & 0 & -1 \\
0 & 0 & 1 & -1
\end{array}\right], \quad P_{0}=\left[\begin{array}{lllll}
-1 & -2 & 2 & 0 & 0 \\
-1 & -2 & 0 & 2 & 0 \\
-1 & -2 & 0 & 0 & 4
\end{array}\right] \text {. }
$$

So, the exponent vectors $l_{i}$ are $l_{0}=(1,2), l_{1}=l_{2}=(2)$ and $l_{3}=(4)$. Accordingly, we obtain the four monomials

$$
T_{0}^{l_{0}}=T_{01} T_{02}^{2}, \quad T_{1}^{l_{1}}=T_{11}^{2}, \quad T_{2}^{l_{2}}=T_{21}^{2}, \quad T_{3}^{l_{3}}=T_{31}^{4} .
$$

We arrive at $r-c=1$ relation $g_{1} \in \mathbb{K}\left[T_{01}, T_{02}, T_{11}, T_{21}, T_{31}\right]$, obtained by computing the following $4 \times 4$ determinant

$$
g_{1}=\operatorname{det}\left[\begin{array}{cccc}
1 & 0 & 0 & -1 \\
0 & 1 & 0 & -1 \\
0 & 0 & 1 & -1 \\
T_{01} T_{02}^{2} & T_{11}^{2} & T_{21}^{2} & T_{31}^{4}
\end{array}\right]=T_{01} T_{02}^{2}+T_{11}^{2}+T_{21}^{2}+T_{31}^{4} .
$$

The canonical basis vectors of the row space of $P_{0}$ are indexed in accordance with the variables $T_{i j}$, that means that we write

$$
e_{01}, e_{02}, e_{11}, e_{21}, e_{31} \in \mathbb{Z}^{5}=\mathbb{Z}^{n_{0}+n_{1}+n_{2}+n_{3}} .
$$


We have $K_{0}=\mathbb{Z}^{5} / \operatorname{im}\left(P_{0}^{*}\right)=\mathbb{Z}^{2} \oplus \mathbb{Z} / 2 \mathbb{Z} \oplus \mathbb{Z} / 2 \mathbb{Z}$. The projection $Q_{0}: \mathbb{Z}^{5} \rightarrow K_{0}$ sending $e_{i j}$ to its class in $K_{0}$ is made concrete by the degree matrix

$$
Q_{0}=\left[Q_{0}\left(e_{i j}\right)\right]=\left[\begin{array}{lllll}
2 & 1 & 2 & 2 & 1 \\
0 & 2 & 2 & 2 & 1 \\
\overline{0} & \overline{0} & \overline{1} & \overline{1} & \overline{0} \\
\overline{0} & \overline{0} & \overline{1} & \overline{0} & \overline{0}
\end{array}\right] .
$$

Consequently, for the initial data $A$ and $P_{0}$ of this example, the resulting $K_{0^{-}}$ graded algebra $R\left(A, P_{0}\right)$ is given by

$$
\mathbb{K}\left[T_{01}, T_{02}, T_{11}, T_{21}, T_{31}\right] /\left\langle T_{01} T_{02}^{2}+T_{11}^{2}+T_{21}^{2}+T_{31}^{4}\right\rangle, \quad \operatorname{deg}\left(T_{i j}\right)=Q_{0}\left(e_{i j}\right) .
$$

We present the basic properties of the graded algebra $R\left(A, P_{0}\right)$. Recall that a grading of a $\mathbb{K}$-algebra $R=\oplus_{K} R_{w}$ by a finitely generated abelian group is effective if the weights $w \in K$ with $R_{w} \neq\{0\}$ generate $K$ as a group and pointed, if $R_{0}=\mathbb{K}$ holds and $R_{w} \neq\{0\} \neq R_{-w}$ is only possible for torsion elements $w \in \mathbb{K}$. Finally, we say that an effective grading is of complexity $c$ if $\operatorname{dim}(R)-\operatorname{rk}(K)=c$ holds.

THEOREM 6.5. Let $R\left(A, P_{0}\right)$ be a $K_{0}$-graded $\mathbb{K}$-algebra arising from Construction 6.3. Then $R\left(A, P_{0}\right)$ is an integral, normal, complete intersection ring satisfying

$$
\operatorname{dim}\left(R\left(A, P_{0}\right)\right)=n+m-r+c, \quad R\left(A, P_{0}\right)^{*}=\mathbb{K}^{*} .
$$

The $K_{0}$-grading of $R\left(A, P_{0}\right)$ is effective, pointed, factorial and of complexity $c$. The variables $T_{i j}, S_{k}$ define pairwise nonassociated $K_{0}$-primes in $R\left(A, P_{0}\right)$, and for $c \geqslant 2$, they define even primes.

The following auxiliary statements for the proof of this theorem are also used later. We begin with discussing the specific nature of the matrix $A$ and its impact on the ideal of relations of $R(A, P)$.

REMARK 6.6. Situation as in Construction 6.3. For any tuple $I=\left(i_{1}, \ldots, i_{c+2}\right)$ of strictly increasing integers from $[0, r]$, consider the matrix

$$
A(I):=\left[a_{i_{1}}, \ldots, a_{i_{c+2}}\right],
$$

Let $w(I) \in \mathbb{K}^{c+2}$ denote the cross product of the rows of $A(I)$ and define a vector $v(I) \in \mathbb{K}^{r+1}$ by putting the entries of $w(I)$ at the right places:

$$
v(I)_{i}:= \begin{cases}w(I)_{j}, & i=i_{j} \text { occurs in } I=\left(i_{1}, \ldots, i_{c+2}\right), \\ 0, & \text { else. }\end{cases}
$$


Then any linearly independent choice of vectors $v\left(I_{1}\right), \ldots, v\left(I_{r-c}\right)$ is a basis for $\operatorname{ker}(A)$. Note that any nonzero $v \in \operatorname{ker}(A)$ has at least $c+2$ nonzero coordinates.

REMARK 6.7. Situation as in Construction 6.3. Every vector $v \in \operatorname{ker}(A) \subseteq \mathbb{K}^{r+1}$ defines a polynomial

$$
g_{v}:=v_{0} T_{0}^{l_{0}}+\cdots+v_{r} T_{r}^{l_{r}} \in\left\langle g_{1}, \ldots, g_{r-c}\right\rangle .
$$

Moreover, if a subset $B \subseteq \operatorname{ker}(A)$ generates $\operatorname{ker}(A)$ as a vector space, then the polynomials $g_{v}, v \in B$, generate the ideal $\left\langle g_{1}, \ldots, g_{r-c}\right\rangle$. In particular, we have

$$
\left\langle g_{1}, \ldots, g_{r-c}\right\rangle=\left\langle g_{v(I)} ; I=\left(i_{1}, \ldots, i_{c+2}\right), 0 \leqslant i_{1}<\cdots<i_{c+2} \leqslant r\right\rangle,
$$

with the tuples $I$ from Remark 6.7. Observe that each $g_{v}, 0 \neq v \in \operatorname{ker}(A)$, has at least $c+2$ of the monomials $T_{i}^{l_{i}}$ and all the $g_{v}$ share the same $K_{0}$-degree.

LEMMA 6.8. Let $R\left(A, P_{0}\right)$ be a graded algebra arising from Construction 6.3.

(i) If we have $l_{i 1}+\cdots+l_{i n_{i}}=1$ for some $i$, then $R\left(A, P_{0}\right)$ is isomorphic to a ring $R\left(A^{\prime}, P_{0}^{\prime}\right)$ with data $r^{\prime}=r-1$ and $c^{\prime}=c$.

(ii) For any generator $T_{i j}$, the factor ring $R\left(A, P_{0}\right) /\left\langle T_{i j}\right\rangle$ is isomorphic to a ring $R\left(A^{\prime}, P_{0}^{\prime}\right)$ with data $r^{\prime}=r-1$ and $c^{\prime}=c-1$.

Proof. To obtain (i), let $A^{\prime}$ be the matrix obtained by deleting the $i$ th column from $A$. Then the respective ideals defined by $A$ and $A^{\prime}$ produce isomorphic rings. Adapting the matrix $P_{0}$ accordingly, gives the desired $P_{0}^{\prime}$.

We show (ii). As elementary row operations on $A$ neither change the required properties of $A$ nor the defining ideal of $R(A, P)$, we may assume that $a_{i 1} \neq$ 0 holds and all other entries of the $i$ th column of $A$ equal zero. Then the matrix $A^{\prime}$ obtained by deleting the first row and the $i$ th column from $A$ satisfies the assumptions of Construction 6.3 with $r^{\prime}=r-1$ and $c^{\prime}=c-1$. Using Remarks 6.6 and 6.7, we see that the ideal defined by $A^{\prime}$ corresponds to the defining ideal of $R\left(A, P_{0}\right) /\left\langle T_{i j}\right\rangle$. Again, adapting the matrix $P_{0}$ accordingly, gives the desired $P_{0}^{\prime}$.

Definition 6.9. Situation as in Construction 6.3. We say that a point $z \in \mathbb{K}^{n+m}$ with coordinates $z_{i j}, z_{k}$ is of

(i) big type, if for every $i=0, \ldots, r$, there is an index $1 \leqslant j_{i} \leqslant n_{i}$ such that $z_{i j_{i}}=0$ holds,

(ii) leaf type, if there is a set $I_{z}=\left\{i_{1}, \ldots, i_{c}\right\}$ of indices $0 \leqslant i_{1}<\cdots<i_{c} \leqslant r$, such that for all $i, j$, we have $z_{i j}=0 \Rightarrow i \in I_{z}$. 
REMARK 6.10. Situation as in Construction 6.3. Consider $\gamma=\mathbb{Q}^{n+m}$, a face $\gamma_{0} \preccurlyeq \gamma$ and the complementary face $\gamma_{0}^{*} \preccurlyeq \gamma$. Then any coordinate $z_{i j}$, $z_{k}$ of $z=z_{\gamma_{0}^{*}} \in \mathbb{K}^{n+m}$ equals zero or one and we have

$$
z_{i j}=0 \Longleftrightarrow e_{i j} \in \gamma_{0}, \quad z_{k}=0 \Longleftrightarrow e_{k} \in \gamma_{0} .
$$

In particular, there is a point of big (leaf) type in $\mathbb{T}^{n+m} \cdot z_{\gamma_{0}^{*}} \subseteq \mathbb{K}^{n+m}$ if and only if all points of this toric orbit are of big (leaf) type. Moreover, in terms of the cone $P_{0}\left(\gamma_{0}^{*}\right)$ with $P_{0}$ from Construction 6.3, we obtain the following characterizations:

(i) $z_{\gamma_{0}^{*}}$ is of big type if and only if $P_{0}\left(\gamma_{0}^{*}\right)=\mathbb{Q}^{r}$ holds,

(ii) $z_{\gamma_{0}^{*}}$ is of leaf type if and only if $P_{0}\left(\gamma_{0}^{*}\right) \neq \mathbb{Q}^{r}$ and $\operatorname{dim}\left(P_{0}\left(\gamma_{0}^{*}\right)\right) \leqslant c$.

Observe that if one of the conditions of (ii) holds, then the image cone $P_{0}\left(\gamma_{0}^{*}\right)$ is generated by at most $r$ vectors from $-\mathbb{1}_{r}, e_{1}, \ldots, e_{r} \in \mathbb{Z}^{r}$ and thus is pointed.

LEMMA 6.11. For $\bar{X}=V\left(g_{1}, \ldots, g_{r-c}\right) \subseteq \mathbb{K}^{n+m}$ from Construction 6.3, we have the following statements.

(i) Every point $z \in \bar{X}$ is either of big type or it is of leaf type.

(ii) Every $z \in \mathbb{K}^{n+m}$ of big type is contained in $\bar{X}$.

(iii) For every $z \in \mathbb{K}^{n+m}$ of leaf type, there is a $t \in \mathbb{T}^{n+m}$ with $t \cdot z \in \bar{X}$.

Proof. To obtain (i), we have to show that any $z \in \bar{X}$ which is not of big type must be of leaf type. Otherwise, there are indices $i_{1}<\cdots<i_{c+1}$ and associated $j_{q}$ with $z_{i_{q} j_{q}}=0$. As $z$ is not of big type, there is at least one index $i_{0}$ with $z_{i_{0} j} \neq 0$ for all $j=1, \ldots, n_{i_{0}}$. Remarks 6.6 and 6.7 provide us with a relation $g \in\left\langle g_{1}, \ldots\right.$, $\left.g_{r-c}\right\rangle$ involving precisely the monomials $T_{i}^{l_{i}}$ for $i=i_{0}, i_{1}, \ldots, i_{c+1}$. Then $g(z)=0$ implies $z_{i_{0} j}=0$ for some $j=1, \ldots, n_{i_{0}}$; a contradiction.

We verify (ii) and (iii). Let $z \in \mathbb{K}^{n+m}$. If $z$ is of big type, then we obviously have $g_{i}(z)=0$ for $i=1, \ldots, r-c$. Thus, $z \in \bar{X}$. Now, assume that $z$ is of leaf type. First consider the case $I_{z}=\{1, \ldots, c\}$. Then, suitably scaling $z_{c+1,1}$, we achieve $g_{1}(z)=0$. Next we scale $z_{c+2,1}$ to ensure $g_{2}(z)=0$, and so on, until we have also $g_{r-c}(z)=0$. Then we have found our $t \in \mathbb{T}^{n+m}$ with $t \cdot z \in \bar{X}$. Given an arbitrary $I_{z}$, Remarks 6.6 and 6.7 yield a suitable system $g_{1}^{\prime}, \ldots, g_{r-c}^{\prime}$ of ideal generators that allows us to argue analogously.

LEMMA 6.12. Situation as in Construction 6.3. Let $\bar{X}=V\left(g_{1}, \ldots, g_{r-c}\right) \subseteq \mathbb{K}^{n+m}$ and denote by $J$ the Jacobian of $g_{1}, \ldots, g_{r-c}$. Then, for any $z \in \bar{X}$, the following statements are equivalent: 
(i) The Jacobian $J(z)$ is not of full rank, that is, we have $\operatorname{rk}(J(z))<r-c$.

(ii) The point $z \in \bar{X}$ is of big type and there are $i_{1}<\cdots<i_{c+2}$ such that each of these $i_{q}$ fulfills one of the subsequent two conditions:

- $z_{i_{q} j_{q}}=0$ and $l_{i_{q} j_{q}} \geqslant 2$ hold for at least one $1 \leqslant j_{q} \leqslant n_{i_{q}}$,

- $z_{i_{q} j}=0$ and $l_{i_{q} j}=1$ hold for at least two $1 \leqslant j \leqslant n_{i_{q}}$.

In particular, the set of points $z \in \bar{X}$ with $J(z)$ not of full rank is of codimension at least $c+1$ in $\bar{X}$.

Proof. Assertion (ii) directly implies the supplement and, by a simple computation, also (i). We are left with proving '(i) $\Rightarrow$ (ii)'. So, let $z \in \bar{X}$ be a point such that $J(z)$ is not of full rank. Then there is a nontrivial linear combination annulating the lines of $J(z)$ :

$$
\eta_{1} \operatorname{grad}\left(g_{1}\right)(z)+\cdots+\eta_{r-c} \operatorname{grad}\left(g_{r-c}\right)(z)=0 .
$$

The corresponding $g:=\eta_{1} g_{1}+\cdots+\eta_{r-c} g_{r-c}$ satisfies $\operatorname{grad}(g)(z)=0$ and is of the form $g=g_{v}$ with a nonzero $v \in \operatorname{ker}(A)$ as in Remark 6.7. The condition $\operatorname{grad}(g)(z)=0$ implies $z_{i j_{i}}=0$ for some $1 \leqslant j_{i} \leqslant n_{i}$ whenever the monomial $T_{i}^{l_{i}}$ shows up in $g$. As observed in Remark 6.7, the polynomial $g$ has at least $c+2$ monomials. Thus, we have $z_{i j_{i}}=0$ for at least $c+2$ different $i$. By Lemma 6.11, the point $z \in \bar{X}$ is of big type. Moreover, the two conditions of (ii) reflect the fact $\operatorname{grad}(g)(z)=0$.

Proof of Theorem 6.5. For $c=1$, the statement is proven in [41, Theorem 10.1 and Proposition 10.7]. So, assume $c \geqslant 2$. First we show that $\bar{X}=V\left(g_{1}\right.$, $\left.\ldots, g_{r-c}\right) \subseteq \mathbb{K}^{n+m}$ is connected. By construction, the quasitorus $H_{0} \subseteq \mathbb{T}^{n+m}$ is the kernel of the homomorphism $\mathbb{T}^{n+m} \rightarrow \mathbb{T}^{r}$ defined by $P_{0}$. Consider the multiplicative one-parameter subgroup $\mathbb{K}^{*} \rightarrow H_{0}, t \mapsto\left(t^{\xi}, t^{\xi}\right)$, where

$$
\zeta=\left(\frac{n_{0} \cdots n_{r} l_{01} \cdots l_{r n_{r}}}{n_{0} l_{01}}, \ldots, \frac{n_{0} \cdots n_{r} l_{01} \cdots l_{r n_{r}}}{n_{r} l_{r n_{r}}}\right) \in \mathbb{T}^{n}, \quad \xi=(1, \ldots, 1) \in \mathbb{T}^{m}
$$

This gives rise to a $\mathbb{K}^{*}$-action on $\bar{X}$ having the origin as an attractive fixed point. Consequently, $\bar{X}$ is connected. Moreover, we can conclude that all invertible functions as well as all $H_{0}$-invariant functions are constant on $\bar{X}$.

Now, Lemma 6.12 allows us to apply Serre's criterion and thus we obtain that $R\left(A, P_{0}\right)$ is an integral, normal, complete intersection. By construction, the $K_{0^{-}}$ grading is effective and as seen above, it is pointed. To obtain factoriality of the $K_{0}$-grading, localize $R\left(A, P_{0}\right)$ by the product over all generators $T_{i j}, S_{k}$, observe 
that the degree zero part of the resulting ring is a polynomial ring and apply [11, Theorem 1.1]. Finally, primality of the generators $T_{i j}$ follows from Lemma 6.8(i).

Now we use the algebras $R\left(A, P_{0}\right)$ obtained by Construction 6.3 to produce general arrangement varieties. The basic idea is to turn $R\left(A, P_{0}\right)$ into a prospective Cox ring via coarsening the grading by $K_{0}=\mathbb{Z}^{n+m} / \operatorname{im}\left(P_{0}^{*}\right)$ to a grading by $K=\mathbb{Z}^{n+m} / \operatorname{im}\left(P^{*}\right)$, where $P$ arises from $P_{0}$ by adding suitable further rows.

Construction 6.13. Let $A$ and $P_{0}$ be input data as in Construction 6.3. Moreover, fix $1 \leqslant s \leqslant n+m-r$ and let $d$ be an integral $s \times(n+m)$ matrix such that the columns $v_{i j}, v_{k}$ of the $(r+s) \times(n+m)$ stack matrix

$$
P:=\left[\begin{array}{c}
P_{0} \\
d
\end{array}\right]
$$

are pairwise different, primitive and generate $\mathbb{Q}^{r+s}$ as a vector space. Consider the factor group $K:=\mathbb{Z}^{n+m} / \operatorname{im}\left(P^{*}\right)$. Then the projection $Q: \mathbb{Z}^{n+m} \rightarrow K$ factors through $Q_{0}$ and we obtain the $K$-graded $\mathbb{K}$-algebra associated with $(A, P)$ :

$$
\begin{aligned}
R(A, P) & :=\mathbb{K}\left[T_{i j}, S_{k}\right] /\left\langle g_{1}, \ldots, g_{r-c}\right\rangle, \\
\operatorname{deg}\left(T_{i j}\right) & :=w_{i j}:=Q\left(e_{i j}\right), \quad \operatorname{deg}\left(S_{k}\right):=w_{k}:=Q\left(e_{k}\right) .
\end{aligned}
$$

Now, let $\Sigma$ be any fan in $\mathbb{Z}^{r+s}$ having precisely the rays through the columns of $P$ as its one-dimensional cones and let $Z$ be the associated toric variety. Then we have a commutative diagram

$$
\begin{aligned}
& V\left(g_{1}, \ldots, g_{r-c}\right)=\bar{X} \subseteq \bar{Z}=\mathbb{Z}^{n+m}
\end{aligned}
$$

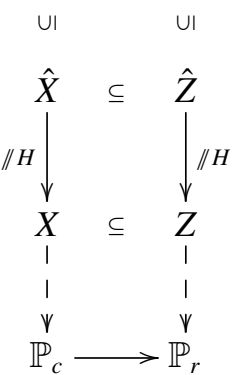

with the quasitorus $H=\operatorname{Spec} \mathbb{K}[K]$, Cox's quotient presentation $\hat{Z} \rightarrow Z$ and the induced quotient $\hat{X} \rightarrow X$, where $\hat{Z}:=\bar{X} \cap \hat{Z}$. The resulting variety $X=X(A, P$, $\Sigma$ ) is normal with dimension, invertible functions, divisor class group and CoX 
ring given by

$$
\operatorname{dim}(X)=s+c, \quad \Gamma\left(X, \mathcal{O}^{*}\right)=\mathbb{K}^{*}, \quad \mathrm{Cl}(X)=K, \quad \mathcal{R}(X)=R(A, P) .
$$

The acting torus $\mathbb{T}_{Z} \subseteq Z$ splits as $\mathbb{T}_{Z}=\mathbb{T}^{r} \times \mathbb{T}^{s}$ and the factor $\mathbb{T}=\left\{\mathbb{1}_{r}\right\} \times \mathbb{T}^{s}$ leaves $X \subseteq Z$ invariant. The induced $\mathbb{T}$-action on $X$ is effective and of complexity

$$
c(X)=c .
$$

Finally, the dashed arrows indicate the maximal orbit quotients for the $\mathbb{T}$-actions on $X$ and $Z$, respectively, and $\mathbb{P}_{c} \subseteq \mathbb{P}_{r}$ is the linear subspace given by

$$
\mathbb{P}_{c}=V\left(h_{1}, \ldots, h_{r-c}\right), \quad h_{t}:=\operatorname{det}\left[\begin{array}{cccc}
a_{0} & \ldots & a_{c} & a_{c+t} \\
U_{0} & \ldots & U_{c} & U_{c+t}
\end{array}\right] \in \mathbb{K}\left[U_{0}, \ldots, U_{r}\right] .
$$

A collection of doubling divisors for the maximal orbit quotient $X--\rightarrow \mathbb{P}_{c}$ is given by the intersections of $\mathbb{P}_{c}$ with the coordinate hyperplanes of $\mathbb{P}_{r}$ which form the general hyperplane arrangement

$$
H_{0}, \ldots, H_{r} \subseteq \mathbb{P}_{c}, \quad H_{i}:=\left\{z \in \mathbb{P}_{c} ; a_{i 0} z_{0}+\cdots+a_{i c} z_{c}=0\right\} .
$$

Proposition 6.14. Let $X=X(A, P, \Sigma)$ arise from Construction 6.13. Consider the explicit variety $Y=\mathbb{P}_{c}$ in $Z_{\Delta}=\mathbb{P}_{r}$ with embedding system $\alpha=\left(f_{0}, \ldots, f_{r}\right)$, where $\Delta$ is the complete fan in $\mathbb{Z}^{r}$ with generator matrix $B=\left[-\mathbb{1}_{r}, \mathbb{E}_{r}\right]$ and

$$
f_{i}=a_{i 0} U_{0}+\cdots+a_{i c} U_{c} \in \mathbb{K}\left[U_{0}, \ldots, U_{c}\right]=\mathcal{R}\left(\mathbb{P}_{c}\right) .
$$

Then the variety $X(A, P, \Sigma) \subseteq Z_{\Sigma}$ equals the variety $X(\alpha, P, \Sigma) \subseteq Z_{\Sigma}$ arising from Construction 3.5. In particular, $X(A, P, \Sigma) \subseteq Z_{\Sigma}$ is an explicit $\mathbb{T}$-variety.

Proof of Construction 6.13 and Proposition 6.14. The fact that $X(A, P$, $\Sigma) \subseteq Z_{\Sigma}$ equals $X(\alpha, P, \Sigma) \subseteq Z_{\Sigma}$ is clear by construction. Observe that, forgetting for the moment about the gradings, we have $R(A, P)=R\left(A, P_{0}\right)$. Thus, Theorem 6.5 ensures that $R(A, P)$ is normal, integral with only constant homogeneous units. Moreover, for $c \geqslant 2$, the generators $T_{i j}$ and $S_{k}$ of $R(A, P)$ are pairwise nonassociated. Being prime and $K$-homogeneous, they are also $K$ prime. For $c=1$, we infer $K$-primality of the generators from [41, Theorem 10.4]. So, $X(A, P, \Sigma) \subseteq Z_{\Sigma}$ satisfies the conditions of Definition 3.8 and hence is an explicit $\mathbb{T}$-variety. This yields in particular the statements on the divisor class group and the Cox ring. The statement on the maximal orbit quotient is due to Proposition 3.16. 
REMARK 6.15. According to Lemma 6.8(i), we may always assume that the defining data $P$ of Construction 6.13 is irredundant in the sense that $l_{i 0}+\cdots+$ $l_{i n_{i}} \geqslant 2$ holds for every $i=0, \ldots, r$. In this case, we also say that $X(A, P, \Sigma)$ is irredundant.

DEFINITION 6.16. By an explicit general arrangement variety we mean a $\mathbb{T}$ variety $X=X(A, P, \Sigma)$ in $Z=Z_{\Sigma}$ arising from Construction 6.13.

EXAmple 6.17. Let $A$ and $P_{0}$ be as in Example 6.4. We enhance $P_{0}$ by an $1 \times 5$ block as follows

$$
P=\left[\begin{array}{l}
P_{0} \\
d
\end{array}\right]=\left[\begin{array}{lllll}
-1 & -2 & 2 & 0 & 0 \\
-1 & -2 & 0 & 2 & 0 \\
-1 & -2 & 0 & 0 & 4 \\
-1 & -3 & 1 & 1 & 1
\end{array}\right]
$$

So, we chose $s=1$. We have $K=\mathbb{Z}^{5} / \operatorname{im}\left(P^{*}\right)=\mathbb{Z} \oplus \mathbb{Z} / 2 \mathbb{Z} \oplus \mathbb{Z} / 2 \mathbb{Z}$ and $Q: \mathbb{Z}^{5} \rightarrow K$ is represented by the degree matrix, having $w_{i j}=Q\left(e_{i j}\right) \in K$ as its columns:

$$
Q=\left[w_{01}, w_{02}, w_{11}, w_{21}, w_{31}\right]=\left[\begin{array}{llllll}
2 & 1 & 2 & 2 & 1 \\
\overline{0} & \overline{0} & \overline{1} & \frac{1}{1} & 0 \\
\overline{0} & \overline{1} & 0 & \frac{1}{1} & 0
\end{array}\right] .
$$

Let $\Sigma$ be the unique complete fan in $\mathbb{Z}^{4}$ with $P$ as its generator matrix. Then we arrive at a projective explicit arrangement variety $X=X(A, P, \Sigma)$ in $Z=Z_{\Sigma}$ with

$$
\operatorname{dim}(X)=3, \quad c(X)=2, \quad \operatorname{Cl}(X)=\mathbb{Z} \oplus \mathbb{Z} / 2 \mathbb{Z} \oplus \mathbb{Z} / 2 \mathbb{Z} .
$$

Moreover, assigning to each generator $T_{i j}$ the divisor class $Q\left(e_{i j}\right)$, we obtain a representation of the Cox ring by homogeneous generators and relations:

$$
\mathcal{R}(X)=\mathbb{K}\left[T_{01}, T_{02}, T_{11}, T_{21}, T_{31}\right] /\left\langle T_{01} T_{02}^{2}+T_{11}^{2}+T_{21}^{2}+T_{31}^{4}\right\rangle .
$$

As a maximal orbit quotient, we have $\pi: X \rightarrow \mathbb{P}_{2}$ and the doubling divisors form the general line configuration in $\mathbb{P}_{2}$ given by

$$
V\left(T_{0}\right), \quad V\left(T_{1}\right), \quad V\left(T_{2}\right), \quad V\left(T_{0}+T_{1}+T_{2}\right) .
$$

THEOREM 6.18. Let $X$ be an $A_{2}$-maximal general arrangement variety. Then $X$ admits a presentation as an explicit general arrangement variety.

Proof. According to Definition 6.1, there is a maximal orbit quotient $\pi: X \rightarrow-$ $Y$ with $Y=\mathbb{P}_{c}$ admitting a general hyperplane arrangement $C_{0}, \ldots, C_{r}$ as a collection of doubling divisors. Then the canonical sections $1_{C_{0}}, \ldots, 1_{C_{r}}$ are 
of degree one in the Cox ring $\mathcal{R}(Y)=\mathbb{K}\left[U_{0}, \ldots, U_{c}\right]$. Suitably enhancing the general hyperplane arrangement $C_{0}, \ldots, C_{r}$, we achieve that $1_{C_{0}}, \ldots, 1_{C_{r}}$ generate $\mathcal{R}(Y)$. Regard $\mathcal{R}(Y)$ as a graded subalgebra of $\mathcal{R}(X)$ as in Corollary 4.2 and let $\alpha=\left(f_{0}, \ldots, f_{q}\right)$ be pairwise nonassociated $\mathrm{Cl}(X)$-prime generators of the Cox ring $\mathcal{R}(X)$ such that the $f_{i}$ lying in $\mathcal{R}(Y)$ are precisely $1_{C_{0}}, \ldots, 1_{C_{r}}$. Then, following the lines of the proof of Theorem 3.10, one reproduces $X$ as an explicit $\mathbb{T}$-variety $X(\alpha, P, \Sigma)$ in $Z=Z_{\Sigma}$. Thus, Proposition 6.14 gives the assertion.

REMARK 6.19. Let $X$ be a general arrangement variety of complexity $c$. Then the torus action of $X$ has $\mathbb{P}_{c}$ as Chow quotient; use [7, Propositions 2.4 and 2.5] for a proof. Using the conversions Remark 3.17 and [46, Theorem 4.8], we see that the general arrangement varieties are precisely the $\mathbb{T}$-varieties arising from a divisorial fan $\Xi$ on a projective space $\mathbb{P}_{c}$ in the sense of [2] such that the prime divisors $D \subseteq \mathbb{P}_{c}$ with nontrivial slices $\Xi_{D}$ form a general hyperplane arrangement in $\mathbb{P}_{c}$.

\section{Examples and first properties}

We discuss examples and study basic structural properties of general arrangement varieties. For instance, we investigate torsion in the divisor class group, describe the canonical class and give a combinatorial characterization of the $X$-cones which in turn leads to the combinatorial smoothness criterion provided by Corollary 7.16. Moreover, we specify constraints on the defining data of an explicit general arrangement variety imposed by conditions on the singularities, preparing the classification performed in Section 9. As a first concrete application, we prove at the end of this section that the smooth projective general arrangement varieties of Picard number one are just the classical smooth projective quadrics; see Proposition 7.23.

We begin with the examples. The first one shows how to realize intrinsic quadrics as explicit general arrangement varieties. Recall from [15] that an intrinsic quadric is a normal projective variety with a Cox ring defined by a single quadratic relation. The intrinsic quadrics form a playground immediately adjacent to the one given by the projective toric varieties, which have a polynomial ring as Cox ring. We mention Bourqui's work [19] proving Manin's conjecture for the full intrinsic quadrics and the classification results on smooth (Fano) intrinsic quadrics of low Picard number in [30] as examples for research in this field.

EXAMPLE 7.1. The normal form for graded quadrics provided by [30, Proposition 2.1] shows that we can represent every intrinsic quadric as an explicit general arrangement variety $X \subseteq Z$ with defining matrix $P$ having left 
upper block

$$
\left[\begin{array}{rrrr}
-l_{0} & l_{1} & & 0 \\
\vdots & \ddots & \\
-l_{0} & 0 & & l_{r}
\end{array}\right], \quad l_{0}=\cdots=l_{q}=(1,1), \quad l_{q+1}=\cdots=l_{r}=(2)
$$

where $-1 \leqslant q \leqslant r$ and the variables $T_{i 1}$ with $i=q+1, \ldots, r$ have pairwise distinct $K$-degrees. Moreover, for the dimension of $X$, the rank of the divisor class group and the complexity of the torus action on $X$, we have

$$
\operatorname{dim}(X)=r-1+s, \quad \operatorname{rk}(\mathrm{Cl}(X))=m+q+2-s, \quad c(X)=r-1 .
$$

In the second example we exhibit a series of general arrangement varieties producing many Fano examples. We pick up these varieties again in Example 7.18, when the necessary methods are available to figure out the smooth Fano varieties.

EXAMPLE 7.2. Fix integers $r>c \geqslant 1$. Consider the product $Z=\mathbb{P}_{r} \times \mathbb{P}_{r}$ and the intersection $X=V\left(g_{1}\right) \cap \cdots \cap V\left(g_{r-c}\right) \subseteq Z$ of the $r-c$ divisors of bidegree $(a, b)$ in $Z$ given by

$$
\begin{aligned}
g_{1} & =\lambda_{1,0} T_{01}^{a} T_{02}^{b}+\lambda_{1,1} T_{11}^{a} T_{12}^{b}+\cdots+\lambda_{1, c} T_{c 1}^{a} T_{c 2}^{b}+T_{c+1,1}^{a} T_{c+1,2}^{b}, \\
& \vdots \\
g_{r-c} & =\lambda_{r-c, 0} T_{01}^{a} T_{02}^{b}+\lambda_{r-c, 1} T_{11}^{a} T_{12}^{b}+\cdots+\lambda_{r-c, c} T_{c 1}^{a} T_{c 2}^{b}+T_{r 1}^{a} T_{r 2}^{b},
\end{aligned}
$$

where $a, b>0$ are coprime integers and any $c+1$ of the vectors $\lambda_{i}=\left(\lambda_{i, 0}, \ldots\right.$, $\left.\lambda_{i, c}\right)$ are linearly independent. Observe that for $r>c+1$, the divisors $V\left(g_{i}\right) \subseteq Z$ are singular. We realize $X \subseteq Z$ as an explicit general arrangement variety. Let $P$ be the stack matrix with upper and lower blocks

$$
\begin{aligned}
P_{0}= & {\left[\begin{array}{rrrr}
-l_{0} & l_{1} & & 0 \\
\vdots & \ddots & \\
-l_{0} & 0 & & l_{r}
\end{array}\right], \quad l_{0}=\cdots=l_{r}=(a, b), } \\
d= & {\left[\begin{array}{rrrr}
-d_{0} & d_{1} & & 0 \\
\vdots & & \ddots & \\
-d_{0} & 0 & & d_{r}
\end{array}\right], \quad d_{0}=\cdots=d_{r}=(v, u), }
\end{aligned}
$$

where $u$ and $v$ are integers with $u a-v b=1$. We claim that there is precisely one complete fan $\Sigma$ with generator matrix $P$ and the associated toric variety $Z=Z_{\Sigma}$ is the product $\mathbb{P}_{r} \times \mathbb{P}_{r}$. Indeed, consider the matrices

$$
\left[\begin{array}{rr}
u \cdot \mathbb{E}_{r}-b \cdot \mathbb{E}_{r} \\
-v \cdot \mathbb{E}_{r} & a \cdot \mathbb{E}_{r}
\end{array}\right], \quad\left[\begin{array}{rrrr}
-\mathbb{1}_{r} & \mathbb{E}_{r} & 0 & 0 \\
0 & 0 & -\mathbb{1}_{r} & \mathbb{E}_{r}
\end{array}\right]
$$


The first one is unimodular and multiplying it from the left to $P$ yields, after suitably renumbering columns, the second one. Now, choosing a suitable $(c+1) \times$ $(r+1)$ matrix $A$, we obtain the above relations as the output of Construction 6.13. Thus, $X=X(A, P, \Sigma)$ is of dimension $r+c$ and comes with an effective $r$-torus action.

We enter the study of structural properties of explicit general arrangement varieties $X \subseteq Z$ as provided by Construction 6.13 . We will freely use the notation fixed there. Our first observation is that there may occur unavoidable torsion in the divisor class group.

Proposition 7.3. Let $X \subseteq Z$ be an explicit general arrangement variety. Then $\mathbb{Z}^{r} / \mathrm{im}\left(P_{0}\right)$ is a finite subgroup of the divisor class group $\mathrm{Cl}(X)$.

Proof. The divisor class group of $X$ equals $K=\mathbb{Z}^{n+m} / \operatorname{im}\left(P^{*}\right)$. Moreover, $\mathbb{Z}^{r} / \operatorname{im}\left(P_{0}\right)$ is the torsion part $K_{0}^{\text {tors }}$ of the factor group $K_{0}=\mathbb{Z}^{n+m} / \operatorname{im}\left(P_{0}^{*}\right)$. Applying the snake Lemma to the exact sequences arising from $P_{0}^{*}$ and $P^{*}$ yields that the kernel of $K_{0} \rightarrow K$ injects into $\mathbb{Z}^{s}$. Consequently, the torsion part $K_{0}^{\text {tors }}$ maps injectively into $K$.

In Remark 6.7, we observed that $R(A, P)$ is a complete intersection ring. Thus, we can apply Proposition 5.10 and obtain the following description of the canonical class.

Proposition 7.4. Let $X \subseteq Z$ be an explicit general arrangement variety of complexity $c(X)=c$. Then the canonical class of $X$ is given in terms of the generator degrees $w_{i j}=\operatorname{deg}\left(T_{i j}\right)$ and $w_{k}=\operatorname{deg}\left(S_{k}\right)$ as

$$
\mathcal{K}_{X}=-\sum_{i=0}^{r} \sum_{j=1}^{n_{i}} w_{i j}-\sum_{k=0}^{r} w_{k}+(r-c) \sum_{j=1}^{n_{0}} l_{0 j} w_{0 j} \in K=\mathrm{Cl}(X) \text {. }
$$

EXAMPLE 7.5. Consider again the explicit general arrangement varieties $X \subseteq Z$ discussed in Example 7.2. The degree matrix $Q$ is

$$
Q=\left[w_{01}, w_{02}, \ldots, w_{r 1}, w_{r 2}\right]=\left[\mathbb{E}_{2}, \ldots, \mathbb{E}_{2}\right] .
$$

Thus, Proposition 5.6 tells us $\operatorname{Eff}(X)=\operatorname{SAmple}(X)=$ cone $\left(e_{1}, e_{2}\right)$. Moreover, the anticanonical class of $X$ is given by

$$
-\mathcal{K}_{X}=(r+1-(r-c) a, r+1-(r-c) b) \in \mathrm{Cl}(X)=\mathbb{Z}^{2}
$$


as we infer from Proposition 7.4. In particular, $X$ is a Fano variety if and only if the following two conditions are satisfied

$$
a<\frac{r+1}{r-c}, \quad b<\frac{r+1}{r-c} .
$$

Recall that in Definition 5.2 we introduced for any explicit variety $X \subseteq Z$ the $X$-cones as those cones $\sigma \in \Sigma$ of the defining fan of $Z$ such that $X$ intersects the corresponding orbit $\mathbb{T}_{Z} \cdot z_{\sigma}$ nontrivially. For explicit general arrangement varieties $X \subseteq Z$, we may determine the $X$-cones in a simple purely combinatorial way.

Definition 7.6. Consider the setting of Construction 6.13 and let $\sigma \in \Sigma$. We say that the cone $\sigma$ is

(i) big (elementary big) if $\sigma$ contains at least (precisely) one column $v_{i j}$ of $P$ for every $i=0, \ldots, r$,

(ii) a leaf cone if there is a set $I_{\sigma}=\left\{i_{1}, \ldots, i_{c}\right\}$ of indices $0 \leqslant i_{1}<\cdots<i_{c} \leqslant r$ such that for any $i$, we have $v_{i j} \in \sigma \Rightarrow i \in I_{\sigma}$.

REMARK 7.7. Situation as in Construction 6.13. Given $\sigma \in \Sigma$, let $\gamma_{0} \preccurlyeq \gamma$ be the corresponding face, that means that $\sigma=P\left(\gamma_{0}^{*}\right)$ holds. Then $\sigma$ is a big (leaf) cone if and only if the toric orbit $\mathbb{T}^{n+m} \cdot z_{\gamma_{0}^{*}} \subseteq \mathbb{K}^{n+m}$ consists of points of big (leaf) type in the sense of Definition 6.9.

Proposition 7.8. Let $X \subseteq Z$ be an explicit general arrangement variety. Then, for every $\sigma \in \Sigma$, the following statements are equivalent.

(i) The cone $\sigma$ is an $X$-cone.

(ii) The cone $\sigma$ is big or a leaf cone.

Proof. Consider the face $\gamma_{0} \preccurlyeq \gamma$ with $P\left(\gamma_{0}^{*}\right)=\sigma$. By Remark 7.7, our $\sigma$ is a big (leaf) cone if and only if $\bar{X}\left(\gamma_{0}\right)$ consists of points of big (leaf) type. The assertion thus follows from Lemma 6.11.

EXAMPLE 7.9. We look again at $X=X(A, P, \Sigma)$ in $Z=Z_{\Sigma}$ from Examples 6.4 and 6.17. Recall that we have

$$
P=\left[v_{01}, v_{02}, v_{11}, v_{21}, v_{31}\right]=\left[\begin{array}{lllll}
-1 & -2 & 2 & 0 & 0 \\
-1 & -2 & 0 & 2 & 0 \\
-1 & -2 & 0 & 0 & 4 \\
-1 & -3 & 1 & 1 & 1
\end{array}\right] \text {. }
$$


Except cone $\left(v_{01}, v_{02}, v_{11}, v_{21}, v_{31}\right)$, every cone generated by some of the $v_{i j}$ occurs in the fan $\Sigma$. In particular, $\Sigma$ has two big cones

$$
\sigma_{1}=\operatorname{cone}\left(v_{01}, v_{11}, v_{21}, v_{31}\right), \quad \sigma_{2}=\operatorname{cone}\left(v_{02}, v_{11}, v_{21}, v_{31}\right),
$$

and six maximal leaf cones

$$
\begin{gathered}
\tau_{1}=\operatorname{cone}\left(v_{01}, v_{02}, v_{11}\right), \quad \tau_{2}=\operatorname{cone}\left(v_{01}, v_{02}, v_{21}\right), \quad \tau_{3}=\operatorname{cone}\left(v_{01}, v_{02}, v_{31}\right), \\
\tau_{4}=\operatorname{cone}\left(v_{11}, v_{21}\right), \quad \tau_{5}=\operatorname{cone}\left(v_{11}, v_{31}\right), \quad \tau_{6}=\operatorname{cone}\left(v_{21}, v_{31}\right) .
\end{gathered}
$$

Thus, by Proposition 7.8 the $X$-cones of $\Sigma$ are $\sigma_{1}, \sigma_{2}$ and the faces of $\tau_{1}, \ldots, \tau_{6}$. This allows us to determine the Picard group $\operatorname{Pic}(X)$. Recall the degree matrix

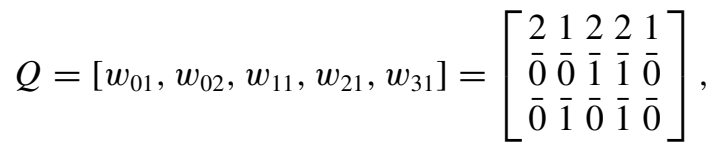

having the generator degrees $w_{i j}=Q\left(e_{i j}\right) \in K=\mathbb{Z} \times \mathbb{Z} / 2 \mathbb{Z} \times \mathbb{Z} / 2 \mathbb{Z}$ as its columns. The $X$-faces corresponding to the $X$-cones $\sigma_{1}, \sigma_{2}, \tau_{1}, \tau_{2}, \tau_{3}$ are

$$
\begin{gathered}
\gamma_{1}=\operatorname{cone}\left(e_{02}\right), \quad \gamma_{2}=\operatorname{cone}\left(e_{02}\right), \\
\eta_{1}=\operatorname{cone}\left(e_{11}, e_{21}\right), \quad \eta_{2}=\operatorname{cone}\left(e_{11}, e_{21}\right), \quad \eta_{3}=\operatorname{cone}\left(e_{21}, e_{31}\right) .
\end{gathered}
$$

Observe that these are precisely the minimal ones among all $X$-faces $\gamma_{0} \preccurlyeq \gamma=$ $\mathbb{Q}^{5} \geqslant 0$. Thus, Proposition 5.6 yields

$$
\operatorname{Pic}(X)=\bigcap_{i=1}^{2} Q\left(\operatorname{lin}_{\mathbb{Q}}\left(\gamma_{i}\right) \cap \mathbb{Z}^{4}\right) \cap \bigcap_{i=1}^{3} Q\left(\operatorname{lin}_{\mathbb{Q}}\left(\eta_{i}\right) \cap \mathbb{Z}^{4}\right)=\mathbb{Z} \cdot(4, \overline{0}, \overline{0}) \subseteq \operatorname{Cl}(X) .
$$

Using Proposition 7.4, we see that $(4, \overline{0}, \overline{0})$ equals the anticanonical class $-\mathcal{K}_{X}$. In particular, $X$ is a Gorenstein Fano threefold.

Big and leaf cones admit also simple characterizations in terms of the geometry of the defining fan of the ambient toric variety.

REMARK 7.10. Consider the setting of Construction 6.13 and let $L \subseteq \mathbb{Z}^{r+s}$ be the kernel of the projection pr: $\mathbb{Z}^{r+s} \rightarrow \mathbb{Z}^{s}$. Then, for any $\sigma \in \Sigma$, the following statements are equivalent.

(i) The cone $\sigma$ is big.

(ii) We have $\operatorname{pr}(\sigma)=\mathbb{Q}^{r}$.

(iii) We have $\sigma \nsubseteq L_{\mathbb{Q}}$ and $\sigma^{\circ} \cap L_{\mathbb{Q}} \neq \emptyset$. 
Moreover, $\sigma \in \Sigma$ is a leaf cone if and only if its image $\operatorname{pr}(\sigma) \subseteq \mathbb{Q}^{r}$ is a pointed cone of dimension at most $c$.

Proposition 7.11. Situation as in Construction 6.13. Let $L \subseteq \mathbb{Z}^{r+s}$ be the kernel of the projection $\mathrm{pr}: \mathbb{Z}^{r+s} \rightarrow \mathbb{Z}^{s}$ and $\Sigma_{L}$ the fan in $\mathbb{Z}^{r+s}$ consisting of all the faces of the cones $\sigma \cap L_{\mathbb{Q}}$, where $\sigma \in \Sigma$. Then the following statements are equivalent.

(i) $\Sigma_{L}$ is a subfan of $\Sigma$.

(ii) $\Sigma$ contains no big cone.

(iii) $\Sigma$ consists of leaf cones.

Proof. The equivalence of (ii) and (iii) is clear. We prove '(i) $\Rightarrow$ (ii)'. Assume that there is a big cone $\sigma \in \Sigma$. Then $\sigma \cap L_{\mathbb{Q}}$ belongs to $\Sigma_{L}$ but not to $\Sigma$ according to Remark 7.10(iii); a contradiction. We turn to '(ii) $\Rightarrow$ (i)'. The task is to show that for every cone $\sigma \in \Sigma$, the intersection $\sigma \cap L_{\mathbb{Q}}$ is a face of $\sigma$. Let $\tau \preccurlyeq \sigma$ be the minimal face containing $\sigma \cap L_{\mathbb{Q}}$. Then $\tau^{\circ} \cap L_{\mathbb{Q}}$ is nonempty. Since $\tau \in \Sigma$ is not big, we can use Remark 7.10(iii) to conclude $\tau \subseteq L_{\mathbb{Q}}$. This means $\sigma \cap L_{\mathbb{Q}}=\tau$.

We use the concrete description of $X$-cones as big cones and leaf cones to study (quasi)smoothness properties of explicit general arrangement varieties $X \subseteq Z$. First, let us define quasismoothness.

Definition 7.12. Let $X \subseteq Z$ be an explicit $\mathbb{T}$-variety. We say that $x \in X$ is a quasismooth point of $X$ if the fiber $p^{-1}(x) \subseteq \hat{X}$ consists of smooth points of $\bar{X}$.

REMARK 7.13. Let $X \subseteq Z$ be an explicit $\mathbb{T}$-variety, $\sigma \in \Sigma$ an $X$-cone and $\gamma_{0} \preccurlyeq \gamma$ the face with $P\left(\gamma_{0}^{*}\right)=\sigma$. Then, for $x \in X(\sigma)$, the intersection $p^{-1}(x) \cap \bar{X}\left(\gamma_{0}\right)$ equals the closed orbit $H \cdot z$ of $p^{-1}(x)$. In particular, $x \in X$ is quasismooth if and only if $z \in \bar{X}$ is smooth. Moreover, $X(\sigma)$ consists of quasismooth points of $X$ if and only if $\bar{X}\left(\gamma_{0}\right)$ consists of smooth points of $\bar{X}$.

Proposition 7.14. Let $X \subseteq Z$ be an explicit general arrangement variety.

(i) For every big cone $\sigma \in \Sigma$, the following statements are equivalent.

(a) There is a quasismooth point of $X$ in the piece $X(\sigma) \subseteq X$.

(b) The piece $X(\sigma) \subseteq X$ consists of quasismooth points of $X$.

(c) Every sequence $0 \leqslant i_{1}<\cdots<i_{c+2} \leqslant r$ admits $1 \leqslant q \leqslant c+2$ and $1 \leqslant j \leqslant n_{i_{q}}$ such that $v_{i_{q} j} \in \sigma, l_{i_{q} j}=1$ and $v_{i_{q} k} \notin \sigma$ for all $k \neq j$. 
(ii) For every leaf cone $\sigma \in \Sigma$, the piece $X(\sigma) \subseteq X$ consists of quasismooth points of $X$.

Proof. According to Remark 7.13, we just have to care about smoothness of the points of $\bar{X}\left(\gamma_{0}\right)$. By Remark 6.7, a point $z \in \bar{X}\left(\gamma_{0}\right)$ is smooth if and only if the Jacobian $J(z)$ of $g_{1}, \ldots, g_{r-c}$ is of full rank. The latter is characterized via Lemma 6.12(ii). In particular, we see that in the case of a leaf cone $\sigma$, all points of $\bar{X}\left(\gamma_{0}\right)$ are smooth, proving (ii). To show (i), let $\sigma$ be big. By the nature of Condition 6.12(ii), there is a smooth point of $\bar{X}$ in $\bar{X}\left(\gamma_{0}\right)$ if and only if every point of $\bar{X}\left(\gamma_{0}\right)$ is smooth in $\bar{X}$. This establishes the equivalence of (a) and (b). The equivalence of (a) and (c) is obtained by negating Condition 6.12(ii) for a point $z$ of big type.

COROLLARY 7.15. Let $X \subseteq Z$ be a quasismooth explicit general arrangement variety. Assume that $P$ is irredundant and let $\sigma=\operatorname{cone}\left(v_{0 j_{0}}+\cdots+v_{r j_{r}}\right)$ be an elementary big cone of $\Sigma$.

(i) We have $l_{i j_{i}} \geqslant 2$ for at most $c+1$ different $i=0, \ldots, r$.

(ii) We have $n_{i}=1$ for at most $c+1$ different $i=0, \ldots, r$.

Combining Proposition 7.14, Remark 7.13 and Proposition 5.5 leads to the following purely combinatorial smoothness criterion for explicit general arrangement varieties.

COROLLARY 7.16. Let $X \subseteq Z$ be an explicit general arrangement variety.

(i) Let $\sigma \in \Sigma$ be a big cone and $\gamma_{0} \preccurlyeq \gamma$ the corresponding $X$-face. Then the following statement are equivalent.

(a) There is a smooth point of $X$ in the piece $X(\sigma)=X\left(\gamma_{0}\right) \subseteq X$.

(b) The piece $X(\sigma)=X\left(\gamma_{0}\right) \subseteq X$ consists of smooth points of $X$.

(c) The cone $\sigma$ is regular and Proposition 7.14(i)(c) holds.

(d) We have $K=Q\left(\operatorname{lin}_{\mathbb{Q}}\left(\gamma_{0}\right) \cap \mathbb{Z}^{n+m}\right)$ and Proposition 7.14(i)(c) holds.

(ii) Let $\sigma \in \Sigma$ be a leaf cone and $\gamma_{0} \preccurlyeq \gamma$ the corresponding $X$-face. Then the following statements are equivalent.

(a) There is a smooth point of $X$ in the piece $X(\sigma)=X\left(\gamma_{0}\right) \subseteq X$.

(b) The piece $X(\sigma)=X\left(\gamma_{0}\right) \subseteq X$ consists of smooth points of $X$.

(c) The cone $\sigma$ is regular.

(d) We have $K=Q\left(\operatorname{lin}_{\mathbb{Q}}\left(\gamma_{0}\right) \cap \mathbb{Z}^{n+m}\right)$. 
EXAMPLE 7.17. Let us continue the discussion of $X \subseteq Z$ from Examples 6.4 and 6.17. In Example 7.9, we determined the maximal $X$-cones: there are two big cones $\sigma_{1}, \sigma_{2}$ and six maximal leaf cones $\tau_{1}, \ldots, \tau_{6}$. Using Corollary 7.16, we see that the associated pieces are precisely those consisting of singular points of $X$. Note that

$$
\overline{X\left(\tau_{i}\right)}=X\left(\sigma_{1}\right) \cup X\left(\tau_{i}\right) \cup X\left(\sigma_{2}\right), \quad i=1,2,3,
$$

are curves, each being the closure of the $\mathbb{T}$-orbit $X\left(\tau_{i}\right)$; use Proposition 5.9. The union over these $\overline{X\left(\tau_{i}\right)}$ is a connected component of the singular locus of $X$. In addition, we have $X\left(\tau_{i}\right)$ for $i=4,5,6$, each consisting of an isolated singularity.

EXAMPLE 7.18. We continue Example 7.2. Suitably renumbering the variables we achieve $a \geqslant b$. We claim that $X$ is smooth if and only if one of the following conditions is satisfied:

(i) $r=c+1, a \geqslant 1$ and $b=1$,

(ii) $r=c+2$ and $a=b=1$.

Indeed, $r \leqslant c+2$ holds, because otherwise the big cone $\sigma \in \Sigma$ generated by all $v_{i j}$ with $i \leqslant r-2$ and $v_{r-11}, v_{r 2}$ yields a singular point in $X(\sigma)$. Now, the elementary big cones of $\Sigma$ are precisely cone $\left(v_{0 j_{0}}, \ldots, v_{r j_{r}}\right)$, where $\left\{j_{0}, \ldots, j_{r}\right\}$ equals $\{1,2\}$, and the claim follows from Corollaries 7.15 and 7.16. In particular we obtain smooth Fano varieties in the cases

(iii) $r=c+1,1 \leqslant a \leqslant r$ and $b=1$,

(iv) $r=c+2$ and $a=b=1$.

Finally, we observe constraints on the defining data of an explicit general arrangement variety arising from local factoriality and $\mathbb{Q}$-factoriality.

Proposition 7.19. Let $X \subseteq Z$ be a locally factorial explicit general arrangement variety, where $P$ is irredundant. Assume that $\Sigma$ consists of leaf cones and each of the sets cone $\left(v_{i 1}\right)+L_{\mathbb{Q}}$ is covered by cones of $\Sigma$. Then $n_{i} \geqslant 2$ holds for $i=0, \ldots, r$.

Proof. Assume that $n_{i}=1$ holds for some $i$. Let $\varrho$ denote the ray through $v_{i 1}$ and consider the cone $\tau:=\varrho+L_{\mathbb{Q}}$. We claim that for every $\sigma \in \Sigma$, the intersection $\tau \cap \sigma$ is a face of $\sigma$. Indeed, as $\Sigma$ consists of leaf cones, the image of $\operatorname{pr}(\sigma)$ under the projection pr: $\mathbb{Q}^{r+s} \rightarrow \mathbb{Q}^{r}$ is a pointed cone, having $\operatorname{pr}(\varrho)$ as an extremal ray. Thus, $\tau=\operatorname{pr}^{-1}(\operatorname{pr}(\varrho))$ cuts out a face from $\sigma$. 
By our assumptions, the above claim implies that $\tau=\varrho+L_{\mathbb{Q}}$ is a union of cones of $\Sigma$. Any cone of $\Sigma \backslash \Sigma_{L}$ contained in $\tau$ is necessarily of the form $\varrho+\sigma_{L}$ $\in \Sigma$ with $\sigma_{L} \in \Sigma_{L}$. We conclude that in particular all the cones $\sigma=\varrho+\sigma_{L}$, where $\operatorname{dim}\left(\sigma_{L}\right)=s$, must belong to $\Sigma$. As $\sigma$ and $\sigma_{L}$ are leaf cones, they are $X$-cones by Proposition 7.8. Thus, Proposition 5.5 yields that $\sigma$ and $\sigma_{L}$ are regular. This implies $l_{i 1}=1$; a contradiction to the assumption that $P$ is irredundant.

COROLLARY 7.20. Let $X \subseteq Z$ be a nontoric, projective, locally factorial explicit general arrangement variety. If $\Sigma$ consists of leaf cones, then the Picard number and the complexity of $X$ satisfy

$$
\rho(X) \geqslant r+3 \geqslant c(X)+4
$$

Proof. Since $X$ is nontoric, we may assume that $P$ is irredundant with $r>$ $c$. Moreover, as $X$ is projective, we may assume that $\Sigma$ is complete. Thus, Proposition 7.19 applies and we obtain $n \geqslant 2 r+2$. Then Corollary 5.12 yields the desired estimate.

Proposition 7.21. Let $X \subseteq Z$ be a $\mathbb{Q}$-factorial explicit general arrangement variety. If $\Sigma$ admits a big cone, then it admits an elementary big cone.

Proof. Let $\sigma \in \Sigma$ be a big cone. Then $\sigma$ is an $X$-cone according to Proposition 7.8. Proposition 5.4 tells us that $\sigma$ is simplicial. Now, any elementary big face of $\sigma$ is as wanted.

COROLlary 7.22. Let $X \subseteq Z$ be a nontoric, projective, locally factorial explicit general arrangement variety. If $X$ is of Picard number $\rho(X) \leqslant c+3$, then $\Sigma$ admits an elementary big cone.

We conclude the section with a closer look at smooth projective general arrangement varieties of Picard number one.

Proposition 7.23. Let $X$ be a nontoric, smooth, projective general arrangement variety of Picard number one. Then $X$ is a quadric $V\left(T_{0}^{2}+\right.$ $\left.\cdots+T_{r}^{2}\right) \subseteq \mathbb{P}_{r}$.

Proof. According to Theorem 6.18, it suffices to consider the explicit general arrangement varieties $X \subseteq Z$. Moreover, we may assume that $P$ is irredundant and $n_{0} \geqslant \cdots \geqslant n_{r}$ holds. Finally, we have $K_{\mathbb{Q}}=\mathbb{Q}$ and may assume that the effective cone of $X$ is $\mathbb{Q} \geqslant 0$. 
First we show that there are no variables of type $S_{k}$ in $R(A, P)$. Otherwise, $\gamma_{1}=\operatorname{cone}\left(e_{1}\right) \preccurlyeq \gamma$ is an $X$-face and thus we find a point $x \in \hat{X}$ having $x_{1}$ as its only nonzero coordinate. By smoothness of $X$, the Jacobian of $g_{1}, \ldots, g_{r-c}$ does not vanish at $x$; see Proposition 5.5. This implies $l_{i 1}+\cdots+l_{i n_{i}}=1$ for some $i$; a contradiction to irredundance of $P$.

According to Corollary 7.22, the fan $\Sigma$ admits an elementary big cone. Proposition 7.15 tells us $n_{0} \geqslant 2$. Thus $\gamma_{0 j}=\operatorname{cone}\left(e_{0 j}\right) \preccurlyeq \gamma$ is an $X$-face. Proposition 5.5 yields that $\operatorname{deg}\left(T_{0 j}\right)$ generates $K$. We conclude $K=\mathbb{Z}$ and $\operatorname{deg}\left(T_{0 j}\right)=1$. Additionally, smoothness of $X\left(\gamma_{01}\right)$ implies that $\operatorname{grad}\left(g_{1}\right)(x) \neq 0$ holds for every point $x \in \bar{X}\left(\gamma_{01}\right)$. We conclude $n_{0}=2$ and $\operatorname{deg}\left(g_{1}\right)=2$. This implies $\operatorname{deg}\left(T_{i j}\right)=1$ and for all $i, j$, we obtain $l_{i j}=1$ or $l_{i j}=2$ according to $n_{i}=2$ or $n_{i}=1$.

Finally, observe that $c=r-1$ holds, that is, there is only one defining relation. Indeed, otherwise, we find generators $g_{1}^{\prime}, \ldots, g_{r-c}^{\prime}$, each involving precisely $c+$ 2 monomials and $g_{r-c}^{\prime}$ all different from $T_{0}^{l_{0}}$. Then the corresponding Jacobian vanishes at any $x \in \bar{X}\left(\gamma_{01}\right)$, showing that $X\left(\gamma_{01}\right)$ is singular. A contradiction.

REMARK 7.24. Consider a smooth, projective explicit general arrangement variety $X \subseteq Z$ of Picard number one with $P$ being irredundant. By Proposition 7.23, the divisor class group $\mathrm{Cl}(X)$ is torsion free. Thus, Proposition 7.3 yields

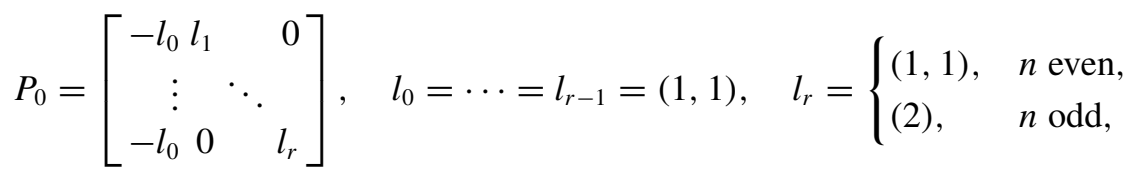

where $n=n_{0}+\cdots+n_{r}$. Moreover, the torus action on $X$ is the action of the maximal torus of $\operatorname{Aut}(X)=\mathrm{O}(n)$. In particular, the torus action on $X$ is of complexity

$$
c= \begin{cases}\frac{n}{2}-2, & n \text { even } \\ \frac{n-1}{2}-1, & n \text { odd }\end{cases}
$$

\section{Proof of the classification Theorem 1.2}

This section is devoted to the proof of Theorem 1.2, which presents all smooth projective general arrangement varieties of true complexity two and Picard number two. Theorem 6.18 allows us to work in the setting of explicit general arrangement varieties $X=X(A, P, \Sigma)$ in $Z=Z_{\Sigma}$. The task is then to specify 
further and further the defining data until we arrive at the list of Theorem 1.2, where we follow a similar strategy as in the case of complexity one [31].

Let us indicate the basic principles behind the proof of Theorem 1.2 and see how smoothness and Picard number two come into the game. Recall from Definition 5.2 the notions of $\bar{X}$-faces and $X$-faces of the orthant $\gamma=\mathbb{Q}_{\geqslant 0}^{n+m}$ and look at the degree map $Q: \mathbb{Z}^{n+m} \rightarrow K=\mathrm{Cl}(X)$, having the image of $P^{*}$ as its kernel. The arguments for establishing the list of Theorem 1.2 rely on the following.

- Due to the explicit nature of the defining relations $g_{1}, \ldots, g_{r-2}$ of $\bar{X}$, we can determine the $\bar{X}$-faces $\gamma_{0} \preccurlyeq \gamma$ and characterize smoothness of the points of the associated pieces $\bar{X}\left(\gamma_{0}\right) \subseteq \bar{X}$ in terms of defining data.

- Since $K_{\mathbb{Q}}=\mathrm{Cl}_{\mathbb{Q}}(X)$ is of dimension two, the positions of the generator degrees $Q\left(e_{i j}\right)$ and $Q\left(e_{k}\right)$ with respect to the ample cone are sufficiently accessible, so that we can figure out possible $X$-faces.

- Smoothness of $X$ is characterized by the fact that for every $X$-face $\gamma_{0} \preccurlyeq \gamma$, the image $Q\left(\gamma_{0} \cap \mathbb{Z}^{n+m}\right)$ generates $K$ as a group and $\bar{X}\left(\gamma_{0}\right)$ consists of smooth points of $\bar{X}$.

As it turns out, the fact that $X$ is of Picard number two allows us to extract enough information from the $\bar{X}$-faces of dimension at most two in order to determine at the end all possibilities for the degree map $Q$ and the ample class of $X$, which in turn lead to the possible matrices $P$ and fans $\Sigma$. In order to obtain that all varieties of Theorem 1.2 are indeed of true complexity two, we have to show that there are no toric examples and no examples of complexity one in the list. This will be done by comparing geometric data, which in the complexity one case we import from [31].

Let us enter the first step toward the list of Theorem 1.2. We will bound the number $r$ which in turn bounds the size of the matrix $A$ and the number of defining relations of the Cox ring $\mathcal{R}(X)=R(A, P)$. Moreover, this step provides first specifications on the numbers $n_{0}, \ldots, n_{r}$ and $m$, which sum up to the number of columns of the matrix $P$. Here comes the precise formulation.

Proposition 8.1. Let $X \subseteq Z$ be a smooth, projective explicit general arrangement variety of Picard number and complexity two. Assume $n_{0} \geqslant \cdots \geqslant n_{r}$ and that $P$ is irredundant. Then we have $\mathrm{Cl}(X)=\mathbb{Z}^{2}$ and one of the following statements holds.

(I) We have $r=3$ and the tuple $\left(n_{0}, n_{1}, n_{2}, n_{3}\right)$ together with the number $m$ fits into one of the cases below, where $n_{0} \geqslant n_{1} \geqslant 3$ : 
(a) $m \geqslant 0$ and $\left(n_{0}, n_{1}, 2,2\right)$,

(b) $m \geqslant 0$ and $\left(n_{0}, 2,2,2\right)$,

(c) $m \geqslant 0$ and $\left(n_{0}, 2,2,1\right)$,

(d) $m=0$ and $(3,2,1,1)$,

(e) $m=0$ and $(3,1,1,1)$, (f) $m \geqslant 0$ and $(2,2,2,2)$,

(g) $m \geqslant 0$ and $(2,2,2,1)$,

(h) $m \geqslant 0$ and $(2,2,1,1)$,

(i) $m>0$ and $(2,1,1,1)$.

(II) We have $r=4$ and $m=0$ and the tuple $\left(n_{0}, n_{1}, n_{2}, n_{3}, n_{4}\right)$ is one of $(2,2,2,2,2),(2,2,2,2,1),(2,2,2,1,1),(2,2,1,1,1)$.

We will obtain this proposition as a consequence of the more general statements, Propositions 8.5, 8.6 and Corollary 8.7, presented and proven below after the necessary preparatory work.

The following Lemma identifies small $\bar{X}$-faces and relates smoothness of the points of $\bar{X}\left(\gamma_{0}\right)$ to data of the defining matrix $P$. It applies to arbitrary explicit general arrangement varieties and generalizes the corresponding statement [31, Lemma 3.9] on the case of complexity one.

LEMMA 8.2. Situation as in Construction 6.13. Consider the orthant $\gamma=\mathbb{Q}_{\geqslant 0}^{n+m}$, its extremal rays $\gamma_{i j}:=\operatorname{cone}\left(e_{i j}\right)$ and $\gamma_{k}:=\operatorname{cone}\left(e_{k}\right)$ and the two-dimensional faces

$$
\gamma_{k_{1}, k_{2}}:=\gamma_{k_{1}}+\gamma_{k_{2}}, \quad \gamma_{i j, k}:=\gamma_{i j}+\gamma_{k}, \quad \gamma_{i_{1} j_{1}, i_{2} j_{2}}:=\gamma_{i_{1} j_{1}}+\gamma_{i_{2} j_{2}} .
$$

(i) All $\gamma_{k}$, respectively $\gamma_{k_{1}, k_{2}}$, are $\bar{X}$-faces and each $\bar{X}\left(\gamma_{k}\right)$, respectively $\bar{X}\left(\gamma_{k_{1}, k_{2}}\right)$, consists of singular points of $\bar{X}$.

(ii) A given $\gamma_{i j}$, respectively $\gamma_{i j, k}$, is an $\bar{X}$-face if and only if $n_{i} \geqslant 2$ holds. In that case, $\bar{X}\left(\gamma_{i j}\right)$, respectively $\bar{X}\left(\gamma_{i j, k}\right)$, consists of smooth points of $\bar{X}$ if and only if $r=c+1, n_{i}=2$ and $l_{i, 3-j}=1$ hold.

(iii) A given $\gamma_{i j_{1}, i j_{2}}$ with $j_{1} \neq j_{2}$ is an $\bar{X}$-face if and only if $n_{i} \geqslant 3$ holds. In that case, $\bar{X}\left(\gamma_{i j_{1}, i j_{2}}\right)$ consists of smooth points of $\bar{X}$ if and only if $r=c+1, n_{i}=3$ and $l_{i j}=1$ for the $j \neq j_{1}, j_{2}$ hold.

(iv) A given $\gamma_{i_{1} j_{1}, i_{2} j_{2}}$ with $i_{1} \neq i_{2}$ is an $\bar{X}$-face if and only if we have either $n_{i_{1}}$, $n_{i_{2}} \geqslant 2$ or $n_{i_{1}}=n_{i_{2}}=1$ and $r=c+1$. In the former case $\bar{X}\left(\gamma_{i_{1} j_{1}, i_{2} j_{2}}\right)$ consists of smooth points of $\bar{X}$ if and only if one of the following holds:

- $r=c+1, n_{i_{t}}=2$ and $l_{i_{t}, 3-j_{t}}=1$ for a $t \in\{1,2\}$,

- $r=c+2, n_{i_{1}}=n_{i_{2}}=2, l_{i_{1}, 3-j_{1}}=l_{i_{2}, 3-j_{2}}=1$.

Proof. Recall that for any face $\gamma_{0} \preccurlyeq \gamma$, we set $\bar{X}\left(\gamma_{0}\right)=\bar{X} \cap \mathbb{T}^{n+m} \cdot z$, where the coordinates $z_{i j}, z_{k}$ of the point $z=z_{\gamma_{0}^{*}} \in \mathbb{K}^{n+m}$ satisfy $z_{i j}=1$ if $e_{i j} \in \gamma_{0}$ 
and $z_{k}=1$ if $e_{k} \in \gamma_{0}$ and equal zero otherwise. Now consider the $\gamma_{0} \preccurlyeq \gamma$ from Assertions (i) to (iii). In these cases, we infer from Lemma 6.11 that $\gamma_{0}$ is an $\bar{X}$ face if and only if $z=z_{\gamma_{0}^{*}}$ is of big type. The latter is trivially satisfied in (i), means $n_{i} \geqslant 2$ in (ii) and $n_{i} \geqslant 3$ in (iii). Moreover, Lemma 6.12 yields the desired characterizations of smoothness. If $\gamma_{0}$ is as in Assertion (iv) with $n_{i_{1}}, n_{i_{2}} \geqslant 2$, then we use Lemmas 6.11 and 6.12 as in the preceding cases. If $\gamma_{0}$ is as in Assertion (iv) one of $n_{i_{1}}, n_{i_{2}}$ equals one, then $z=z_{\gamma_{0}^{*}}$, then Lemma 6.11 that tells us that $\gamma_{0}$ is an $\bar{X}$-face if and only if $z=z_{\gamma_{0}^{*}}$ is of leaf type, which amounts to $n_{i_{1}}=n_{i_{2}}=1$ and $r=c+1$.

Observe that the above statements (iii), (iv) and (v) depend on the complexity $c$. To proceed, we take a look at the ample cone and the position of generator degrees of the Cox ring. Propositions 5.4 and 5.6 lead to the following picture.

REMARK 8.3. Let $X \subseteq Z$ be a projective explicit general arrangement variety with divisor class group $\mathrm{Cl}(X)$ of rank two. Then the effective cone of $X$ is of dimension two and decomposes as

$$
\operatorname{Eff}(X)=\tau^{+} \cup \tau_{X} \cup \tau^{-},
$$

where $\tau_{X} \subseteq \operatorname{Eff}(X)$ is the ample cone, $\tau^{+}, \tau^{-}$are closed cones not intersecting $\tau_{X}$ and $\tau^{+} \cap \tau^{-}$consists of the origin. Due to $\tau_{X} \subseteq \operatorname{Mov}(X)$, each of the cones $\tau^{+}$ and $\tau^{-}$contains at least two of the weights

$$
w_{i j}=\operatorname{deg}\left(T_{i j}\right)=Q\left(e_{i j}\right), \quad w_{k}=\operatorname{deg}\left(S_{k}\right)=Q\left(e_{k}\right) .
$$

Moreover, for every $\bar{X}$-face $\{0\} \neq \gamma_{0} \preccurlyeq \gamma$ precisely one of the following inclusions holds:

$$
Q\left(\gamma_{0}\right) \subseteq \tau^{+}, \quad \tau_{X} \subseteq Q\left(\gamma_{0}\right)^{\circ}, \quad Q\left(\gamma_{0}\right) \subseteq \tau^{-} .
$$

The $X$-faces are exactly the $\bar{X}$-faces $\gamma_{0} \preccurlyeq \gamma$ with $\tau_{X} \subseteq Q\left(\gamma_{0}\right)^{\circ}$. Note that the ample cone $\tau_{X}$ is of dimension two if and only if $X$ is $\mathbb{Q}$-factorial.

The following Lemma is a direct generalization of [31, Lemma 3.11] and provides first constraints for the possible positions of the generator degrees with respect to the ample cone.

LEMMA 8.4. Let $X \subseteq Z$ be a projective explicit general arrangement variety with divisor class group of rank two.

(i) Suppose that $X$ is $\mathbb{Q}$-factorial. Then $w_{k} \notin \tau_{X}$ holds for all $1 \leqslant k \leqslant m$ and for all $0 \leqslant i \leqslant r$ with $n_{i} \geqslant 2$ we have $w_{i j} \notin \tau_{X}$, where $1 \leqslant j \leqslant n_{i}$. 
(ii) Suppose that $X$ is quasismooth, $m>0$ holds and there is $0 \leqslant i_{1} \leqslant r$ with $n_{i_{1}} \geqslant 3$. Then the $w_{i j}, w_{k}$ with $n_{i} \geqslant 3, j=1, \ldots, n_{i}$ and $k=1, \ldots, m$ lie either all in $\tau^{+}$or all in $\tau^{-}$.

(iii) Suppose that $X$ is quasismooth and there is $0 \leqslant i_{1} \leqslant r$ with $n_{i_{1}} \geqslant 4$. Then the $w_{i j}$ with $n_{i} \geqslant 4$ and $j=1, \ldots, n_{i}$ lie either all in $\tau^{+}$or all in $\tau^{-}$.

(iv) Suppose that $X$ is quasismooth and there exist $0 \leqslant i_{1}<i_{2} \leqslant r$ with $n_{i_{1}}$, $n_{i_{2}} \geqslant 3$. Then the $w_{i j}$ with $n_{i} \geqslant 3, j=1, \ldots, n_{i}$ lie either all in $\tau^{+}$or all in $\tau^{-}$.

(v) Suppose that $X$ is quasismooth. Then $w_{1}, \ldots, w_{m}$ lie either all in $\tau^{+}$or all in $\tau^{-}$.

Proof. We prove (i). By Lemma 8.2(i) and (ii), all rays $\gamma_{k}$ and all rays $\gamma_{i j}$ with $n_{i} \geqslant 2$ are $\bar{X}$-faces. By $\mathbb{Q}$-factoriality, $\tau_{X} \subseteq K_{\mathbb{Q}}$ is of dimension two which excludes $\tau_{X} \subseteq Q\left(\gamma_{k}\right)^{\circ}$ and $\tau_{X} \subseteq Q\left(\gamma_{i j}\right)^{\circ}$. Taking $\gamma_{0}=\gamma_{k}$ and $\gamma_{0}=\gamma_{i j}$ in Remark 8.3 gives the claim. We show (ii). By Lemma 8.2(i) and (ii), all $\gamma_{k}$ and all $\gamma_{i j}, \gamma_{i j, k}$ with $n_{i} \geqslant 3$ are $\bar{X}$-faces and the corresponding pieces in $\bar{X}$ consist of singular points of $\bar{X}$. By quasismoothness, none of these $\bar{X}$-faces is an $X$-face. For $\gamma_{0}=\gamma_{i_{1} 1}$, Remark 8.3 yields $w_{i_{1} 1} \in \tau^{+}$or $w_{i_{1} 1} \in \tau^{-}$, say $w_{i_{1} 1} \in \tau^{+}$. Applying Remark 8.3 to $\gamma_{0}=\gamma_{i j, k}$ gives $w_{k}, w_{i j} \in \tau^{+}$for $k=1, \ldots, m$ and all $i$ with $n_{i} \geqslant 3$ and $j=1, \ldots, n_{i}$. Assertions (iii), (iv) and (v) are seen by similar arguments.

Proposition 8.5. Let $X \subseteq Z$ be a nontoric projective quasismooth explicit general arrangement variety with divisor class group of rank two, where $P$ is irredundant and $n_{0} \geqslant \cdots \geqslant n_{r}$. Assume that $m>0$ holds and $\Sigma$ admits an elementary big cone.

(i) We have $r=c+1$ and are in one of the following situations:

(a) We have $n_{0}=2$ and there exist indices $i$ and $j$ such that $n_{i}=2$ holds and $\gamma_{i j, k}$ is an $X$-face for all $k$.

(b) We have $n_{0} \geqslant 3$ and there exist indices $i_{1} \neq i_{2}$ and $j_{1}, j_{2}$ such that $n_{i_{1}}=n_{i_{2}}=2$ holds and $\gamma_{i_{1} j_{1}, k}, \gamma_{i_{2} j_{2}, k}$ are $X$-faces for all $k$.

(ii) Assume $c=2$. Then we have $r=3$ and the constellation of the $n_{i}$ is $\left(n_{0}\right.$, $\left.n_{1}, 2,2\right),\left(n_{0}, 2,2,2\right),\left(n_{0}, 2,2,1\right)(2,2,2,2),(2,2,2,1),(2,2,1,1)$ or $(2$, $1,1,1)$, where $n_{0} \geqslant n_{1} \geqslant 3$.

Proof. Due to Lemma 8.4(v), we may assume $w_{1}, \ldots, w_{m} \in \tau^{+}$. As $X$ is nontoric we have at least one relation $g_{1}$. Thus, $r \geqslant c+1$ holds and Proposition 7.15(ii) 
yields $n_{0} \geqslant 2$. Lemma 8.4(i) says that none of the $w_{i j}$ with $n_{i} \geqslant 2$ lies in $\tau_{X}$. Moreover, at least one of the $w_{i j}$ with $n_{i} \geqslant 2$ lies in $\tau^{-}$; otherwise, since all relations $g_{i}$ share the same degree, we had $w_{i 1} \in \tau^{+}$for all $i$ with $n_{i}=1$, meaning that $\tau^{-}$contains no weights at all; a contradiction. In particular, if $n_{0}=2$ holds, then there exists a $w_{i j} \in \tau^{-}$with $n_{i}=2$ and all $\gamma_{i j, k}$ are $X$-faces. Assume $n_{0} \geqslant 3$. Then Lemma 8.4(ii) yields $w_{i j} \in \tau^{+}$whenever $n_{i} \geqslant 3$. Moreover, because all relations $g_{i}$ have the same degree, $w_{i j} \in \tau^{+}$holds for all $i$ with $n_{i}=1$. Since $\tau^{-}$ contains at least two weights, we find $i_{1}, i_{2}$ and $j_{1}, j_{2}$ with $n_{i_{1}}=n_{i_{2}}=2$ and $w_{i_{1} j_{1}}$, $w_{i_{2} j_{2}} \in \tau^{-}$. Note that all $\gamma_{i_{1} j_{1}, k}, \gamma_{i_{2} j_{2}, k}$ are $X$-faces. Now, Lemma 8.2(ii) yields $r=c+1$. Thus, Assertion (i) is proven. Assertion (ii) is a direct consequence.

Proposition 8.6. Let $X \subseteq Z$ be a nontoric projective quasismooth explicit general arrangement variety with divisor class group of rank two, where $P$ is irredundant and $n_{0} \geqslant \cdots \geqslant n_{r}$. Assume that $m=0$ holds and $\Sigma$ admits an elementary big cone.

(i) We are in one of the following situations:

(a) We have $r=c+1, n_{0}=3>n_{1}$ and there exists an index $j$ such that $\gamma_{01,0 j}$ is an $X$-face.

(b) We have $r=c+1$ and there exist indices $0 \leqslant i_{1}<i_{2}$ with $n_{i_{1}}=n_{i_{2}}=2$ and indices $j_{0}, j_{2}$ such that $\gamma_{0 j_{0}, i_{2} j_{2}}$ is an $X$-face.

(c) We have $r=c+2$ and $n_{0}=n_{1}=2$ and there exist indices $0<i_{1}$ and $j_{0}, j_{1}$ such that $\gamma_{0 j_{0}, i_{1} j_{1}}$ is an $X$-face.

(ii) Assume $c=2$. Then the constellation of the $n_{i}$ is one of the following, where $n_{0} \geqslant n_{1} \geqslant 3$ holds:

$$
\begin{array}{ll}
r=3: & \left(n_{0}, n_{1}, 2,2\right),\left(n_{0}, 2,2,2\right),\left(n_{0}, 2,2,1\right),(3,2,1,1),(3,1,1,1), \\
& (2,2,2,2),(2,2,2,1),(2,2,1,1) . \\
r=4: \quad & (2,2,2,2,2),(2,2,2,2,1),(2,2,2,1,1),(2,2,1,1,1) .
\end{array}
$$

Proof. Only for the first assertion, there is something to show. As $X$ is nontoric we have at least one relation $g_{1}$ and conclude $r \geqslant c+1$. Moreover, Proposition 7.15(ii) yields $n_{0} \geqslant 2$. Finally, Lemma 8.4(i) shows that none of the $w_{i j}$ with $n_{i} \geqslant 2$ lies in $\tau_{X}$. We distinguish the following cases.

First, let $n_{0} \geqslant 4$ or $n_{0}=n_{1}=3$. By Assertions (iii) and (iv) of Lemma 8.4, we may assume $w_{i j} \in \tau^{+}$for all $i$ with $n_{i} \geqslant 3$. Then $w_{i j} \in \tau^{+}$holds as well for all $i$ with $n_{i}=1$. Since $\tau^{-}$contains at least two weights, there are $i_{1}<i_{2}$ and $j_{1}, j_{2}$ with $n_{i_{1}}=n_{i_{2}}=2$ and $w_{i_{1} j_{1}}, w_{i_{2} j_{2}} \in \tau^{-}$. Observe that $\gamma_{01, i_{2} j_{2}}$ is an $X$-face. Moreover, Lemma 8.2(iv) shows $r=c+1$. We arrive at Case (b) of (i). 
Next, let $n_{0}=3>n_{1}$. If all weights $w_{0 j}$ lie either in $\tau^{+}$or in $\tau^{-}$, then we can argue as above and end up in Case (b) of (i). Otherwise, $w_{01}$ and some $w_{0 j}$ for $j=2,3$ lie on different sides of $\tau_{X}$. Then $\gamma_{01,0 j}$ is an $X$-face. Lemma 8.2(iii) yields $r=c+1$ and we are in Case (a) of (i).

Finally, let $n_{0}=2$. The common degree of $g_{1}, \ldots, g_{r-c}$ and hence all $w_{i j}$ with $n_{i}=1$ lie in precisely one of the cones $\tau^{+}, \tau^{-}$or $\tau_{X}$, where we may assume that this is not $\tau^{-}$. Then no pair $w_{i 1}, w_{i 2}$ lies in $\tau^{-}$. As there must be at least two weights in $\tau^{-}$, we conclude $n_{1}=2$ and find the desired $\gamma_{0 j_{0}, 1 j_{1}}$. Lemma 8.2(iv) yields $r \leqslant c+2$. Thus, we are in one of the Cases (b) or (c) of (i).

COROLLARY 8.7. Let $X$ be a smooth projective general arrangement variety of Picard number two. Then we have $\operatorname{Cl}(X)=\operatorname{Pic}(X)=\mathbb{Z}^{2}$.

Proof. We may assume that $X \subseteq Z$ is explicit. Corollary 7.22 tells us that $\Sigma$ admits an elementary big cone. Thus Propositions 8.5 and 8.6 provide a twodimensional $X$-face $\gamma_{0} \preccurlyeq \gamma$. According to Proposition 5.5, the two weights stemming from $\gamma_{0}$ generate $K$ as a group. This implies $\mathrm{Cl}(X) \cong K \cong \mathbb{Z}^{2}$.

Proof of Proposition 8.1. According to Corollary 7.22, the fan $\Sigma$ admits an elementary big cone. Thus, Propositions 8.5(ii) and 8.6(ii) apply. Together with Corollary 8.7 they provide the desired statements.

In order to show that Theorem 1.2 lists all smooth projective general arrangement varieties of true complexity two, we have to go through the cases of Proposition 8.1. After some further preparation, we will treat exemplarily Cases 8.1(I)(a) and (b). The detailed discussion of the remaining cases is given in [70, Section 6.1].

REMARK 8.8. Let $X \subseteq Z$ be a smooth, projective explicit general arrangement variety of Picard number two. Corollary 8.7 ensures $\mathrm{Cl}(X)=\mathbb{Z}^{2}$ and we will write

$$
\begin{gathered}
\operatorname{deg}\left(T_{i j}\right)=Q\left(e_{i j}\right)=w_{i j}=\left(x_{i j}, y_{i j}\right) \in \mathbb{Z}^{2}, \\
\operatorname{deg}\left(T_{k}\right)=Q\left(e_{k}\right)=w_{k}=\left(x_{k}, y_{k}\right) \in \mathbb{Z}^{2}
\end{gathered}
$$

for the weights. Moreover, the (common) degree of the relations $g_{1}, \ldots, g_{r-c}$ will be denoted as $\operatorname{deg}\left(g_{i}\right)=\mu=\left(\mu_{1}, \mu_{2}\right) \in \mathbb{Z}^{2}$. Recall that for each $i=0, \ldots, r$ we have

$$
\mu_{1}=\sum_{j=1}^{n_{i}} l_{i j} x_{i j}, \quad \mu_{2}=\sum_{j=1}^{n_{i}} l_{i j} y_{i j} .
$$


Consider the decomposition of the effective cone $\operatorname{Eff}(X)=\tau^{-} \cup \tau_{X} \cup \tau^{+}$from Remark 8.3. Choosing names suitably, we can fix the following orientation:

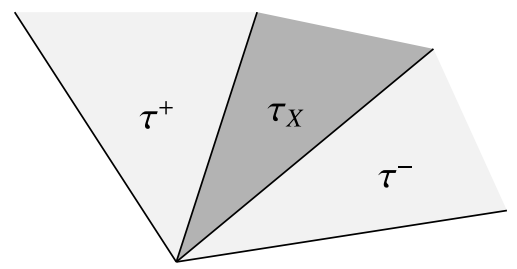

If $\left(w, w^{\prime}\right)$ is a positively oriented pair in $\mathbb{Q}^{2}$, for instance $w \in \tau^{-}$and $w^{\prime} \in \tau^{+}$, then $\operatorname{det}\left(w, w^{\prime}\right)$ is positive. If, furthermore, $w, w^{\prime}$ are the weights stemming from a two-dimensional $X$-face $\gamma_{0} \preccurlyeq \gamma$, then we have $\operatorname{det}\left(w, w^{\prime}\right)=1$ by Proposition 5.5. In that case, we can achieve

$$
w=(1,0), \quad w^{\prime}=(0,1)
$$

by a suitable unimodular coordinate change on $\mathbb{Z}^{2}$. Then $w^{\prime \prime}=\left(x^{\prime \prime}, 1\right)$ holds whenever $w, w^{\prime \prime}$ stems from a two-dimensional $X$-face and, similarly, $w^{\prime \prime}=$ $\left(1, y^{\prime \prime}\right)$ holds whenever $w^{\prime \prime}, w^{\prime}$ stems from a two-dimensional $X$-face.

LEMMA 8.9. In the situation of Proposition 8.1, consider the case $r=3, m \geqslant 0$ and $n_{0} \geqslant 3>n_{1}=n_{2}=2 \geqslant n_{3}$. Then the following constellation of weights cannot occur:

$$
w_{01}, \ldots, w_{0 n_{0}}, w_{12}, w_{22} \in \tau^{+}, \quad w_{11}, w_{21} \in \tau^{-} .
$$

Proof. We may assume $w_{02}, \ldots, w_{0 n_{0}}, w_{21} \in \operatorname{cone}\left(w_{01}, w_{11}\right)$. Applying Remark 8.8 first to the $X$-face $\gamma_{01,11}$ and then to all $\gamma_{01,21}, \gamma_{22,11}, \gamma_{0 j, 11}$, $\gamma_{i, 11} \in \operatorname{rlv}(X)$, where $j=1, \ldots, n_{0}$ and $i=1, \ldots, m$, turns the degree matrix $Q$ into the shape

$$
Q=\left[\begin{array}{cccc|cc|cc|ccc||ccc}
0 & x_{02} & \ldots & x_{0 n_{0}} & 1 & x_{12} & 1 & x_{22} & x_{31} & \ldots & x_{3 n_{1}} & x_{1} & \ldots & x_{m} \\
1 & 1 & \ldots & 1 & 0 & y_{12} & y_{21} & 1 & y_{31} & \ldots & y_{3 n_{1}} & 1 & \ldots & 1
\end{array}\right],
$$

where $x_{0 j} \geqslant 0$ and $y_{21} \geqslant 0$. Moreover, $\gamma_{01,11}, \gamma_{01,21} \in \operatorname{rlv}(X)$ implies $l_{12}=l_{22}=1$ due to Lemma 8.2(iv). Using $\gamma_{21,12} \in \operatorname{rlv}(X)$ we infer $y_{12}=1+y_{21} x_{12}$ from $\operatorname{det}\left(w_{21}, w_{12}\right)=1$ and, by the shape of $Q$, obtain

$$
3 \leqslant l_{01}+\cdots+l_{0 n_{0}}=\mu_{2}=y_{12}=1+y_{21} x_{12} .
$$

We conclude $x_{12}>0$. Using $\gamma_{0 j, 21} \in \operatorname{rlv}(X)$ gives $\operatorname{det}\left(w_{21}, w_{0 j}\right)=1$ and thus $x_{0 j} y_{21}=0$. As the effective cone of $X$ is pointed, $w_{21} \in \tau^{-}$implies $y_{21}>0$. We arrive at $x_{0 j}=0$ and thus $\mu_{1}=0=l_{11}+x_{12}$. This contradicts to the fact that $l_{11}$ and $x_{12}$ are strictly positive. 
We are ready to establish the list of Theorem 1.2. As announced, we restrict ourselves here to the discussion of two cases of Proposition 8.1 and refer to [70, Section 6.1] for the remaining ones.

Case 8.1(I)(a). We have $r=3, m \geqslant 0$ and $n_{0} \geqslant n_{1} \geqslant 3>n_{2}=n_{3}=2$. This setting allows no examples satisfying the assumptions of Theorem 1.2.

Proof. By Assertions (iv) and (ii) of Lemma 8.4, we may assume that the weights $w_{01}, \ldots, w_{0 n_{0}}, w_{11}, \ldots, w_{1 n_{1}}$ and $w_{1}, \ldots, w_{m}$ all lie in $\tau^{+}$. At least two other weights lie in $\tau^{-}$. Renumbering suitably, we arrive at $w_{21}, w_{31} \in \tau^{-}$and $w_{22}$, $w_{32} \in \tau^{+}$because of $\mu \in \tau^{+}$. Thus, Lemma 8.9 gives the assertion.

Case 8.1(I)(b). We have $r=3, m \geqslant 0$ and $n_{0} \geqslant 3>n_{1}=n_{2}=n_{3}=2$. This gives the varieties Nos. 1 and 2 of Theorem 1.2.

Proof. We claim that each of $\tau^{+}$and $\tau^{-}$contains weights from $w_{01}, \ldots, w_{0 n_{0}}$. Otherwise, due to Lemma 8.4(i), we may assume that all $w_{0 j}$ lie in $\tau^{+}$. If $m>0$ holds, Lemma 8.4(ii) yields $w_{1}, \ldots, w_{m} \in \tau^{+}$. As $\tau^{-}$contains at least two weights, we can achieve $w_{11}, w_{21} \in \tau^{-}$and $w_{12}, w_{22} \in \tau^{+}$by suitable renumbering; note that $w_{i 1}, w_{i 2} \in \tau^{-}$is not possible for $i=1,2,3$ because of $\mu \in \tau^{+}$. Lemma 8.9 then verifies the claim.

By the claim, we may assume $w_{01}, w_{02} \in \tau^{+}$and $w_{03} \in \tau^{-}$. Lemma 8.4(ii) shows $m=0$ and Lemma 8.4(iii) gives $n_{0}=3$. There must be at least one more weight in $\tau^{-}$, say $w_{11}$. Applying Lemma 8.2(iii) to $\gamma_{0 j, 03} \in \operatorname{rlv}(X)$ we obtain $l_{01}=$ $l_{02}=1$. Moreover, looking at suitable $X$-faces $\gamma_{0 j, i_{2} j_{2}}$ and using Lemma 8.2(iv), we obtain

$$
l_{11}=l_{12}=l_{21}=l_{22}=l_{31}=l_{32}=1 .
$$

We may assume $w_{02} \in \operatorname{cone}\left(w_{01}, w_{03}\right)$. Then, applying Remark 8.8 to $\gamma_{01,03} \in$ $\operatorname{rlv}(X)$ and afterward to $\gamma_{01,11}, \gamma_{02,03} \in \operatorname{rlv}(X)$ we achieve that our degree matrix looks as follows:

$$
Q=\left[\begin{array}{ccc|cc|cc|cc}
0 & x_{02} & 1 & 1 & x_{12} & x_{21} & x_{22} & x_{31} & x_{32} \\
1 & 1 & 0 & y_{11} & y_{12} & y_{21} & y_{22} & y_{31} & y_{32}
\end{array}\right] .
$$

Note that we have $x_{02} \geqslant 0$ because of $w_{02} \in \operatorname{cone}\left(w_{01}, w_{03}\right)$. We distinguish the following three cases according to the possible positions of the weights $w_{21}$ and $w_{22}$.

We have $w_{21}, w_{22} \in \tau^{-}$. Then $\mu \in \tau^{-}$holds and we may assume $w_{31} \in \tau^{-}$. Moreover, we have $\gamma_{01,21}, \gamma_{01,22}, \gamma_{01,31} \in \operatorname{rlv}(X)$ and conclude

$$
x_{21}=x_{22}=x_{31}=1, \quad \mu=(2,2), \quad x_{12}=x_{22}=x_{32}=1 .
$$


The determinants corresponding to $\gamma_{02,21}, \gamma_{02,22} \in \operatorname{rlv}(X)$ both equal one, which implies $y_{21} x_{02}=0$ and $y_{22} x_{02}=0$. Because of $y_{21}+y_{22}=\mu_{2}=2$, we obtain

$$
x_{02}=0, \quad l_{03}=\mu_{1}=2 .
$$

The considerations performed so far determine the defining relation $g_{1}$ and, up to integers $a_{1}, a_{2}, a_{3}$, also the degree matrix $Q$ as

$$
\begin{gathered}
g_{1}=T_{01} T_{02} T_{03}^{2}+T_{11} T_{12}+T_{21} T_{22}+T_{31} T_{32}, \\
Q=\left[\begin{array}{lll|cc|cc|cc}
0 & 0 & 1 & 1 & 1 & 1 & 1 & 1 & 1 \\
1 & 1 & 0 & a_{1} & 2-a_{1} & a_{2} & 2-a_{2} & a_{3} & 2-a_{3}
\end{array}\right] .
\end{gathered}
$$

We claim that all $w_{i j}$, where $i=1,2,3$, lie in $\tau^{-}$. This means that we have to show $w_{12}, w_{32} \in \tau^{-}$. Otherwise, if $w_{12} \in \tau^{+}$holds, then $\gamma_{03,12} \in \operatorname{rlv}(X)$ leads to

$$
1=\operatorname{det}\left(w_{03}, w_{12}\right)=a_{1} \text {. }
$$

This implies $w_{11}=w_{21} \in \tau^{-} \cap \tau^{+}$, which is impossible. Analogously, one excludes $w_{32} \in \tau^{+}$. Thus, we may assume $a_{1} \leqslant a_{2} \leqslant a_{3}$ and $a_{i} \geqslant 2-a_{i}$. Consequently, $a_{i} \geqslant 1$ holds and we obtain

$$
\operatorname{SAmple}(X)=\overline{\tau_{X}}=\operatorname{cone}\left(\left(1, a_{3}\right),(0,1)\right) .
$$

We have $w_{21}, w_{22} \in \tau^{+}$. Thus, we have $\mu \in \tau^{+}$and thus $w_{12} \in \tau^{+}$. This means that $\gamma_{03,12}, \gamma_{03,21}, \gamma_{21,22}$ are $X$-faces and we can conclude

$$
y_{12}=y_{21}=y_{22}=1, \quad \mu_{2}=2, \quad y_{11}=1 .
$$

Looking at the determinants associated with $\gamma_{02,11}, \gamma_{11,21}, \gamma_{11,22} \in \operatorname{rlv}(X)$ we see $x_{02}=x_{21}=x_{22}=0$. This gives $l_{03}=\mu_{1}=x_{21}+x_{22}=0$; a contradiction.

We have $w_{21} \in \tau^{-}$and $w_{22} \in \tau^{+}$. Then we may assume $w_{31} \in \tau^{-}$and $w_{32} \in \tau^{+}$, as otherwise, up to renumbering, we are in one of the preceding cases. Remark 8.8 applied to $\gamma_{01,21}, \gamma_{01,31}, \gamma_{03,22}, \gamma_{03,32} \in \operatorname{rlv}(X)$ and $\mu_{2}=2$ yield

$$
x_{21}=x_{31}=y_{22}=y_{32}=1, \quad y_{21}=y_{31}=1 .
$$

We claim $y_{11} \neq 0$. Otherwise, $y_{12}=\mu_{2}=2$. This $\operatorname{implies} \operatorname{det}\left(w_{03}, w_{12}\right)=2$, hence $\gamma_{03,12} \notin \operatorname{rlv}(X)$ and thus $w_{12} \in \tau^{-}$. Then $\gamma_{01,12} \in \operatorname{rlv}(X)$ leads to $x_{12}=1$ and $\mu_{1}=2$. Thus $w_{22}=(1,1)=w_{21} \in \tau^{-}$, contradicting the setup. Now, $y_{11} \neq 0$ yields

$$
x_{02}=x_{22}=x_{32}=0, \quad \mu=(1,2), \quad l_{03}=1, \quad x_{12}=0
$$


due to $\gamma_{11,02}, \gamma_{11,22}, \gamma_{11,32} \in \operatorname{rlv}(X)$ and homogeneity of the relation $g_{1}$. We conclude $w_{12}=\left(0, y_{12}\right) \in \tau^{+}$and $\gamma_{03,12} \in \operatorname{rlv}(X)$ shows $y_{12}=1$. Finally, $y_{11}=\mu_{2}-y_{12}=1$ holds. For the relation, the degree matrix and the ample cone, this means

$$
\begin{gathered}
g_{1}=T_{01} T_{02} T_{03}+T_{11} T_{12}+T_{21} T_{22}+T_{31} T_{32}, \\
Q=\left[\begin{array}{lll|l|l|l|l|ll}
0 & 0 & 1 & 1 & 0 & 1 & 0 & 1 & 0 \\
1 & 1 & 0 & 1 & 1 & 1 & 1 & 1 & 1
\end{array}\right], \\
\text { SAmple }(X) \stackrel{\tau_{X}}{=}=\operatorname{cone}((0,1),(1,1))
\end{gathered}
$$

Finally, Theorem 1.2 claims that the listed varieties are all of true complexity two. The following Proposition establishes this statement. As before, we present an example case; the full discussion will be made available elsewhere.

Proposition 8.10. Each of the varieties listed in Theorem 1.2 is of true complexity two, that is, does not admit torus actions of lower complexity.

Proof. First observe that each of the varieties listed in Theorem 1.2 has a singular total coordinate space and hence is not toric. Thus, we have to show that none of the varieties from Theorem 1.2 is isomorphic to a smooth nontoric variety of Picard number two with torus action of complexity one, which in turn are all given in [31, Theorem 1.1]. We do this exemplarily for the variety $X$ listed as No. 5 in our Theorem 1.2. Recall that Cox ring, degree matrix and an ample class of $X$ are

$$
\begin{aligned}
R & =\mathbb{K}\left[T_{1}, \ldots, T_{8}, S_{1}, \ldots, S_{m}\right] /\left\langle T_{1} T_{2}+T_{3}^{2} T_{4}+T_{5}^{2} T_{6}+T_{7}^{2} T_{8}\right\rangle, \quad m \geqslant 0, \\
Q & =\left[\begin{array}{cccccccc|cc|c}
0 & 2 b+1 & b & 1 & b & 1 & b & 1 & 1 & \ldots & 1 \\
1 & 1 & 1 & 0 & 1 & 0 & 1 & 0 & 0 & \ldots & \ldots
\end{array}\right], \quad b \geqslant 0, \\
u & =(2 b+2,1) .
\end{aligned}
$$

The total coordinate space $\operatorname{Spec}(R)$ of $X$ is of dimension $m+7$ with singular locus of codimension 4. Computing these data also for the varieties from [31, Theorem 1.1], we see that $X$ can be isomorphic at most to varieties from Nos. 4, 6, 10, 11 or 12 of [31, Theorem 1.1]. We now go through these cases.

Assume that $X$ is isomorphic to the variety [31, Theorem 1.1, No. 4], which we denote by $X^{\prime}$. Cox ring, degree matrix and an ample class of $X^{\prime}$ are given by

$$
\begin{aligned}
R^{\prime} & =\mathbb{K}\left[T_{1}, \ldots, T_{6}, S_{1}, \ldots, S_{m^{\prime}}\right] /\left\langle T_{1} T_{2}^{l_{2}}+T_{3} T_{4}^{l_{4}}+T_{5} T_{6}^{l_{6}}\right\rangle, \quad m^{\prime} \geqslant 0, \\
Q^{\prime} & =\left[\begin{array}{llllll|ccc}
0 & 1 & a_{1} & 1 & a_{2} & 1 & c_{1} & \ldots & c_{m^{\prime}} \\
1 & 0 & 1 & 0 & 1 & 0 & 1 & \ldots & 0
\end{array}\right], \begin{array}{l}
0 \leqslant a_{1} \leqslant a_{2}, \\
c_{1} \leqslant \ldots \leqslant c_{m^{\prime}}, \\
1 \leqslant l_{2}=a_{1}+l_{4}=a_{2}+l_{6} b,
\end{array} \\
u^{\prime} & =\left(\max \left(a_{2}, c_{m}^{\prime}\right)+1,1\right) .
\end{aligned}
$$


The total coordinate space $\operatorname{Spec}\left(R^{\prime}\right)$ of $X^{\prime}$ is of dimension $m^{\prime}+5$ and the codimension of its singular locus equals 5 minus the number of $i$ with $l_{i} \geqslant 2$. Consequently, we obtain

$$
m^{\prime}=m+2, \quad l_{4}=l_{6}=1, \quad l_{2}=a_{1}+1=a_{2}+1 \geqslant 2, \quad a_{1}=a_{2} .
$$

We write $w_{i}$ for the $i$ th column of $Q$ and denote by $\mu_{i}$ the number of times it shows up as a column of $Q$. Analogously, we define $w_{i}^{\prime}$ and $\mu_{i}^{\prime}$. Then we have

$$
\mu_{1} \in\{1,4\}, \quad \mu_{4}=3+m, \quad \mu_{2}^{\prime}=3, \quad \mu_{1}^{\prime} \leqslant 1+m^{\prime} .
$$

Observe that $w_{1}, w_{4}$ are the primitive generators of the extremal rays of the effective cone of $X$ and $w_{4}$ is a semiample class, whereas $w_{1}$ is not semiample. Moreover, $w_{2}^{\prime}$ is a semiample primitive generator of the effective cone of $X^{\prime}$. We conclude

$$
3+m=\mu_{4}=\operatorname{dim}\left(R_{w_{4}}\right)=\operatorname{dim}\left(R_{w_{2}^{\prime}}^{\prime}\right)=\mu_{2}^{\prime}=3 .
$$

Thus, $m=0$ and $m^{\prime}=2$ hold. Comparing the multiplicities $\operatorname{dim}\left(R_{w}\right)$ and $\operatorname{dim}\left(R_{w^{\prime}}^{\prime}\right)$ for $w$ and $w^{\prime}$ being the primitive generators differing from $(1,0)$ of the respective effective, moving and semiample cones of $X$ and $X^{\prime}$, we obtain

$$
b, c_{1}, c_{2}>0, \quad \mu_{1}^{\prime}=\mu_{1}=1 \quad b=a_{1}=a_{2}=c_{1}<c_{2}=2 b+1 .
$$

But then the anticanonical class $-\mathcal{K}_{X}=(3 a+3,3)$ is divisible by 3 , whereas $-\mathcal{K}_{X^{\prime}}=(4 b+3,3)$ is not; a contradiction.

Assume that $X$ is isomorphic to a variety $X^{\prime}$ as in [31, Theorem 1.1, No. 6]. Here, Cox ring, the degree matrix and an ample class look as follows:

$$
\begin{aligned}
R^{\prime} & =\mathbb{K}\left[T_{1}, \ldots, T_{6}, S_{1}, \ldots, S_{m^{\prime}}\right] /\left\langle T_{1} T_{2}+T_{3} T_{4}+T_{5}^{2} T_{6}\right\rangle, \quad m^{\prime} \geqslant 1, \\
Q^{\prime} & =\left[\begin{array}{cccccc|ccc}
0 & 2 a_{3}+1 & a_{1} & a_{2} & a_{3} & 1 & 1 & \ldots & 1 \\
1 & 1 & 1 & 1 & 1 & 0 & 0 & \ldots & 0
\end{array}\right], \begin{array}{l}
a_{1}, a_{2}, a_{3} \geqslant 0, \\
a_{1}<a_{2}, \\
a_{1}+a_{2}=2 a_{3}+1,
\end{array} \\
u^{\prime} & =\left(2 a_{3}+2,1\right) .
\end{aligned}
$$

The dimension of the total coordinate space $\operatorname{Spec}\left(R^{\prime}\right)$ of $X^{\prime}$ equals $m^{\prime}+5$ and hence $m^{\prime}=m+2$ must hold. As before, let $w_{i}$ be the $i$ th column of $Q$ and $\mu_{i}$ the number of times it shows up as a column of $Q$. Define $w_{i}^{\prime}$ and $\mu_{i}^{\prime}$ analogously. We obtain

$$
\mu_{1} \in\{1,4\}, \quad \mu_{4}=\mu_{6}^{\prime}=m+3, \quad \mu_{1}^{\prime} \in\{1,2,3\} .
$$

For $X$ as well as for $X^{\prime}$, we find precisely one semiample primitive generator of the effective cone, namely $w_{4}$ and $w_{6}^{\prime}$. Consequently we obtain

$$
1=\mu_{1}=\mu_{1}^{\prime}, \quad a_{1}, a_{2}, a_{3}>0 .
$$


Comparing the multiplicities $\operatorname{dim}\left(R_{w}\right)$ and $\operatorname{dim}\left(R_{w^{\prime}}^{\prime}\right)$ for $w$ and $w^{\prime}$ being the primitive generators differing from $(1,0)$ of the effective, movable and semiample cones of $X$ and $X^{\prime}$, we arrive at $a_{1}=a_{2}=a_{3}=b$, which contradicts, for instance, $a_{1}<a_{2}$.

Assume that $X$ is isomorphic to the variety $X^{\prime}$ as in [31, Theorem 1.1, No. 10]. In this case, the Cox ring, the degree matrix and an ample class of $X^{\prime}$ are given as

$$
\begin{aligned}
R^{\prime} & =\mathbb{K}\left[T_{1}, \ldots, T_{5}, S_{1}, \ldots, S_{m^{\prime}}\right] /\left\langle T_{1} T_{2}+T_{3} T_{4}+T_{5}^{2}\right\rangle, \quad m^{\prime} \geqslant 1, \\
Q^{\prime} & =\left[\begin{array}{lllll|lll}
1 & 1 & 1 & 1 & 1 & 0 & \ldots & 0 \\
0 & 2 & 1 & 1 & 1 & 1 & \ldots & 0 \\
u^{\prime} & =(2,1) .
\end{array}\right.
\end{aligned}
$$

Let $w_{i}, w_{i}^{\prime}$ and $\mu_{i}, \mu_{i}^{\prime}$ be as before. Then $w_{4}$ and $w_{1}^{\prime}$ are the only semiample primitive generators of the effective cones of $X$ and $X^{\prime}$, respectively. Thus, we obtain $1=\mu_{1}^{\prime}=\mu_{4}=3+m$; a contradiction.

Assume that $X$ is isomorphic to the variety $X^{\prime}$ as in [31, Theorem 1.1, No. 11]. The Cox ring, the degree matrix and an ample class of $X^{\prime}$ are then given by

$$
\begin{aligned}
R^{\prime} & =\mathbb{K}\left[T_{1}, \ldots, T_{5}, S_{1}, \ldots, S_{m^{\prime}}\right] /\left\langle T_{1} T_{2}+T_{3} T_{4}+T_{5}^{2}\right\rangle, \quad m^{\prime} \geqslant 2, \\
Q^{\prime} & =\left[\begin{array}{lllll|llll}
1 & 1 & 1 & 1 & 1 & 0 & a_{2} & \ldots & a_{m^{\prime}} \\
0 & 0 & 0 & 0 & 0 & 1 & 1 & \ldots & \ldots
\end{array}\right], \quad \begin{array}{l}
0 \leqslant a_{2} \leqslant \cdots \leqslant a_{m^{\prime}}, \\
a_{m^{\prime}}>0,
\end{array} \\
u^{\prime} & =\left(a_{m^{\prime}}+1,1\right) .
\end{aligned}
$$

With $w_{i}, w_{i}^{\prime}$ and $\mu_{i}, \mu_{i}^{\prime}$ as before, we see that, again, $w_{4}, w_{1}^{\prime}$ are the only semiample primitive generators of the effective cones of $X, X^{\prime}$, respectively, and conclude

$$
5=\mu_{1}^{\prime}=\mu_{4}=3+m \text {. }
$$

This implies $m=2$. Thus, $\operatorname{Spec}(R)$ is of dimension $7+m=9$. Consequently, $\operatorname{Spec}\left(R^{\prime}\right)$ is of dimension $9=4+m^{\prime}$, showing $m^{\prime}=5$. Looking for $R$ and $R^{\prime}$ at the homogeneous components of degrees $2 w_{4}$ and $2 w_{1}^{\prime}$, respectively, we arrive at a contradiction:

$$
15=\operatorname{dim}\left(R_{2 w_{4}}\right)=\operatorname{dim}\left(R_{2 w_{1}^{\prime}}^{\prime}\right)=14
$$

Finally, assume that $X$ is isomorphic to the variety $X^{\prime}$ as in [31, Theorem 1.1, No. 12]. The Cox ring, the degree matrix and an ample class of the latter are given as

$$
\begin{aligned}
R^{\prime} & =\mathbb{K}\left[T_{1}, \ldots, T_{5}, S_{1}, \ldots, S_{m^{\prime}}\right] /\left\langle T_{1} T_{2}+T_{3} T_{4}+T_{5}^{2}\right\rangle, \quad m^{\prime} \geqslant 2, \\
Q^{\prime} & =\left[\begin{array}{cccccc|ccc}
0 & 2 a_{3} & a_{1} & a_{2} & a_{3} & 1 & 1 & \ldots & 1 \\
1 & 1 & 1 & 1 & 1 & 0 & 0 & \ldots & 0
\end{array}\right], \quad \begin{array}{l}
0 \leqslant a_{1} \leqslant a_{3} \leqslant a_{2}, \\
a_{1}+a_{2}=2 a_{3}, \\
u^{\prime}
\end{array}=\left(2 a_{3}+1,1\right) .
\end{aligned}
$$


Comparing the primitive generators $w, w^{\prime}$ and the corresponding multiplicities $\operatorname{dim}\left(R_{w}\right), \operatorname{dim}\left(R_{w}^{\prime}\right)$ of the effective, moving and semiample cones of $X$ and $X^{\prime}$, we arrive at

$$
m^{\prime}=3+m, \quad 0<b, \quad 0<a_{1}=a_{2}=a_{3} .
$$

Now, comparing the determinants of the Mori chambers of $X$ and $X^{\prime}$ leads to a contradiction: we obtain

$b=\operatorname{det}\left(w_{3}, w_{1}\right)=\operatorname{det}\left(w_{5}^{\prime}, w_{2}\right)=a_{3}, \quad b+1=\operatorname{det}\left(w_{2}, w_{3}\right)=\operatorname{det}\left(w_{2}^{\prime}, w_{3}^{\prime}\right)=a_{3}$.

REMARK 8.11. According to Theorem 6.18, the varieties from Theorem 1.2 can be represented as explicit general arrangement varieties $X(A, P, \Sigma)$ in the sense of Definition 6.16. The following table provides the defining data $P$ and $\Sigma$, where we denote the columns of $P$ by $v_{1}, \ldots, v_{n+m}$ and set $\sigma_{i, j}:=\operatorname{cone}\left(v_{k} ; k \neq i\right.$, $k \neq j)$.

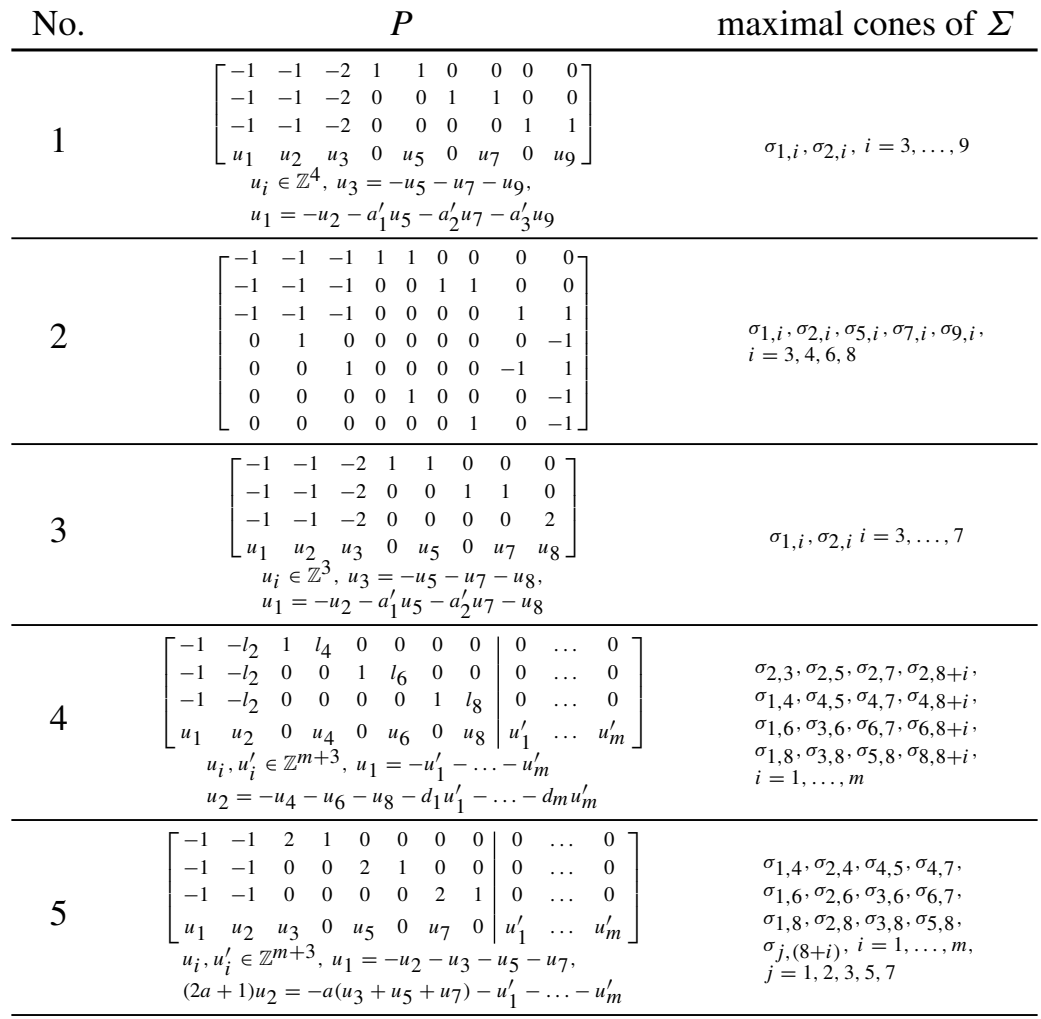




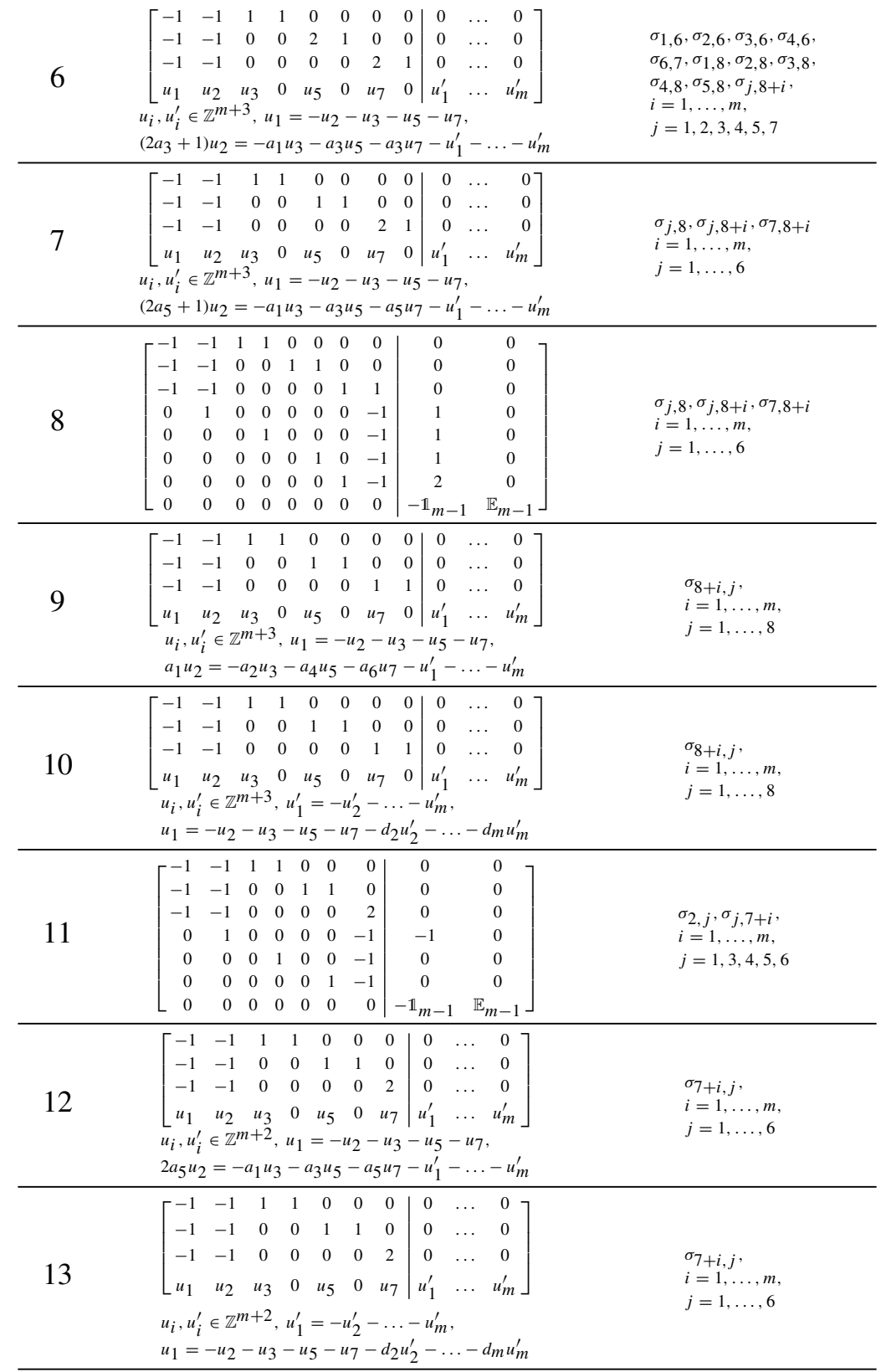




\section{Fano arrangement varieties and their geometry}

In this section, we present the Fano and almost Fano varieties from Theorem 1.2 and discuss geometric properties of the Fano varieties. The key is the description of the anticanonical class of an explicit general arrangement variety $X \subseteq Z$ given by

$$
-\mathcal{K}_{X}=\sum_{i=0}^{r} \sum_{j=1}^{n_{i}} w_{i j}+\sum_{k=0}^{r} w_{k}-(r-c) \sum_{j=1}^{n_{0}} l_{0 j} w_{0 j} \in K=\mathrm{Cl}(X),
$$

where $c=c(X)$ is the complexity and $w_{i j}=\operatorname{deg}\left(T_{i j}\right)$ as well as $w_{k}=\operatorname{deg}\left(T_{k}\right)$ are the Cox ring generator degrees; see Proposition 7.4. Going through the list of Theorem 1.2 and picking the cases with $-\mathcal{K}_{X}$ lying in the ample cone, we obtain the following.

THEOREM 9.1. Every smooth Fano general arrangement variety of true complexity two and Picard number two is isomorphic to precisely one of the following varieties $X$, specified by their Cox ring $\mathcal{R}(X)$ and the matrix $\left[w_{1}, \ldots\right.$, $w_{r}$ ] of generator degrees $w_{i} \in \mathrm{Cl}(X)=\mathbb{Z}^{2}$.

\begin{tabular}{|c|c|c|c|c|}
\hline No. & $\mathcal{R}(X)$ & {$\left[w_{1}, \ldots, w_{r}\right]$} & $-\mathcal{K}_{X}$ & $\operatorname{dim}(X)$ \\
\hline 1 & $\frac{\mathbb{K}\left[T_{1}, \ldots, T_{9}\right]}{\left\langle T_{1} T_{2} T_{3}^{2}+T_{4} T_{5}+T_{6} T_{7}+T_{8} T_{9}\right\rangle}$ & 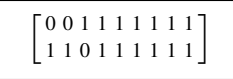 & {$\left[\begin{array}{l}5 \\
6\end{array}\right]$} & 6 \\
\hline 2 & $\frac{\mathbb{K}\left[T_{1}, \ldots, T_{9}\right]}{\left\langle T_{1} T_{2} T_{3}+T_{4} T_{5}+T_{6} T_{7}+T_{8} T_{9}\right\rangle}$ & {$\left[\begin{array}{lllllllll}0 & 0 & 1 & 0 & 1 & 0 & 1 & 0 \\
1 & 1 & 0 & 1 & 1 & 1 & 1 & 1 & 1\end{array}\right]$} & {$\left[\begin{array}{l}3 \\
6\end{array}\right]$} & 6 \\
\hline 3 & $\frac{\mathbb{K}\left[T_{1}, \ldots, T_{8}\right]}{\left\langle T_{1} T_{2} T_{3}^{2}+T_{4} T_{5}+T_{6} T_{7}+T_{8}^{2}\right\rangle}$ & 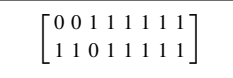 & {$\left[\begin{array}{l}4 \\
5\end{array}\right]$} & 5 \\
\hline $4 A$ & $\begin{array}{c}\frac{\mathbb{K}\left[T_{1}, \ldots, T_{8}, S_{1}, \ldots, S_{m}\right]}{\left\langle T_{1} T_{2}^{3}+T_{3} T_{4}+T_{5} T_{6}+T_{7} T_{8}\right\rangle} \\
m \geqslant 0\end{array}$ & {$\left[\begin{array}{llllllll|lll}0 & 1 & 2 & 2 & 1 & 1 & 2 & \ldots & 2 \\
1 & 0 & 1 & 0 & 1 & 0 & 1 & 0 & 1 & \ldots & 1\end{array}\right]$} & {$\left[\begin{array}{c}7+2 m \\
3+m\end{array}\right]$} & $m+5$ \\
\hline $4 B$ & $\begin{array}{c}\frac{\mathbb{K}\left[T_{1}, \ldots, T_{8}, S_{1}, \ldots, S_{m}\right]}{\left\langle T_{1} T_{2}^{3}+T_{3} T_{4}^{2}+T_{5} T_{6}^{2}+T_{7} T_{8}^{2}\right\rangle} \\
m \geqslant 0\end{array}$ & {$\left[\begin{array}{lllllllllll}0 & 1 & 1 & 1 & 1 & 1 & 1 & 1 & \ldots & 1 \\
1 & 0 & 1 & 0 & 1 & 0 & 1 & 0 & 1 & \ldots & 1\end{array}\right]$} & {$\left[\begin{array}{l}4+m \\
3+m\end{array}\right]$} & $m+5$ \\
\hline $4 C$ & $\begin{array}{c}\frac{\mathbb{K}\left[T_{1}, \ldots, T_{8}, S_{1}, \ldots, S_{m}\right]}{\left\langle T_{1} T_{2}^{2}+T_{3} T_{4}^{2}+T_{5} T_{6}+T_{7} T_{8}\right\rangle} \\
m \geqslant 0\end{array}$ & {$\left[\begin{array}{lllllllllll}0 & 1 & 0 & 1 & 1 & 1 & 1 & 1 & \ldots & 1 \\
1 & 0 & 1 & 0 & 1 & 0 & 1 & 0 & 1 & \ldots & 1\end{array}\right]$} & {$\left[\begin{array}{l}4+m \\
3+m\end{array}\right]$} & $m+5$ \\
\hline
\end{tabular}


$4 D \quad \frac{\mathbb{K}\left[T_{1}, \ldots, T_{8}, S_{1}, \ldots, S_{m}\right]}{\left\langle T_{1} T_{2}^{2}+T_{3} T_{4}+T_{5} T_{6}+T_{7} T_{8}\right\rangle}$ $m \geqslant 0$

$\mathbb{K}\left[T_{1}, \ldots, T_{8}, S_{1}, \ldots, S_{m}\right]$

$4 E \quad\left\langle T_{1} T_{2}^{3}+T_{3} T_{4}^{3}+T_{5} T_{6}^{3}+T_{7} T_{8}^{3}\right\rangle$ $m \geqslant 0$

$4 F \quad \frac{\mathbb{K}\left[T_{1}, \ldots, T_{8}, S_{1}, \ldots, S_{m}\right]}{\left\langle T_{1} T_{2}^{2}+T_{3} T_{4}^{2}+T_{5} T_{6}^{2}+T_{7} T_{8}^{2}\right\rangle}$ $m \geqslant 0$

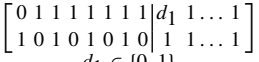
$d_{1} \in\{0,1\}$

$$
\left[\begin{array}{c}
5+m-1+d_{1} \\
3+m
\end{array}\right] m+5
$$

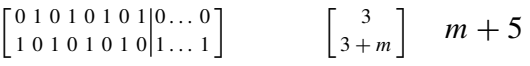

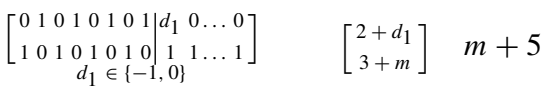

\begin{tabular}{|c|c|c|c|c|}
\hline $4 G$ & $\begin{array}{c}\frac{\mathbb{K}\left[T_{1}, \ldots, T_{8}, S_{1}, \ldots, S_{m}\right]}{\left\langle T_{1} T_{2}+T_{3} T_{4}+T_{5} T_{6}+T_{7} T_{8}\right\rangle} \\
m \geqslant 0\end{array}$ & 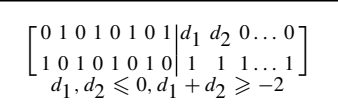 & {$\left[\begin{array}{c}3+d_{1}+d_{2} \\
3+m\end{array}\right]$} & $m+5$ \\
\hline 5 & $\begin{array}{c}\frac{\mathbb{K}\left[T_{1}, \ldots, T_{8}, S_{1}, \ldots, S_{m}\right]}{\left\langle T_{1} T_{2}+T_{3}^{2} T_{4}+T_{5}^{2} T_{6}+T_{7}^{2} T_{8}\right\rangle} \\
m \geqslant 1\end{array}$ & {$\left[\begin{array}{cccccccc|ccc}0 & 2 a+1 & a & 1 & a & 1 & a & 1 & 1 & \ldots & 1 \\
1 & 1 & 1 & 0 & 1 & 0 & 1 & 0 & 0 & \ldots & 0\end{array}\right]$} & {$\left[\begin{array}{c}3 a+3+m \\
3\end{array}\right]$} & $m+5$ \\
\hline 6 & $\begin{array}{c}\frac{\mathbb{K}\left[T_{1}, \ldots, T_{8}, S_{1}, \ldots, S_{m}\right]}{\left\langle T_{1} T_{2}+T_{3} T_{4}+T_{5}^{2} T_{6}+T_{7}^{2} T_{8}\right\rangle} \\
m \geqslant 1\end{array}$ & 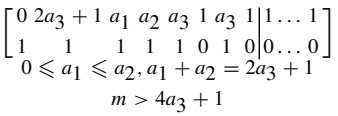 & {$\left[\begin{array}{c}4 a_{3}+3+m \\
4\end{array}\right]$} & $m+5$ \\
\hline
\end{tabular}

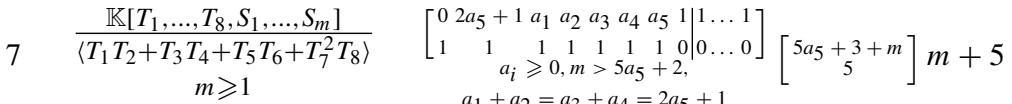
$a_{1}+a_{2}=a_{3}+a_{4}=2 a_{5}+1$

\begin{tabular}{|c|c|c|c|c|}
\hline 8 & $\begin{array}{c}\frac{\mathbb{K}\left[T_{1}, \ldots, T_{8}, S_{1}, \ldots, S_{m}\right]}{\left\langle T_{1} T_{2}+T_{3} T_{4}+T_{5} T_{6}+T_{7} T_{8}\right\rangle} \\
1 \leqslant m \leqslant 5\end{array}$ & {$\left[\begin{array}{cccccccc|ccc}0 & 0 & 0 & 0 & 0 & 0 & -1 & 1 & 1 & \ldots & 1 \\
1 & 1 & 1 & 1 & 1 & 1 & 1 & 0 & \ldots & 0\end{array}\right]$} & {$\left[\begin{array}{c}m \\
6\end{array}\right]$} & $m+5$ \\
\hline 9 & $\begin{array}{c}\frac{\mathbb{K}\left[T_{1}, \ldots, T_{8}, S_{1}, \ldots, S_{m}\right]}{\left\langle T_{1} T_{2}+T_{3} T_{4}+T_{5} T_{6}+T_{7} T_{8}\right\rangle} \\
m \geqslant 2\end{array}$ & 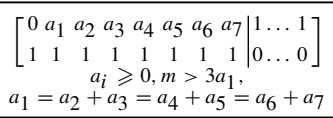 & {$\left[\begin{array}{c}3 a_{1}+m \\
6\end{array}\right]$} & $m+5$ \\
\hline 10 & $\begin{array}{c}\frac{\mathbb{K}\left[T_{1}, \ldots, T_{8}, S_{1}, \ldots, S_{m}\right]}{\left\langle T_{1} T_{2}+T_{3} T_{4}+T_{5} T_{6}+T_{7} T_{8}\right\rangle} \\
m \geqslant 2\end{array}$ & 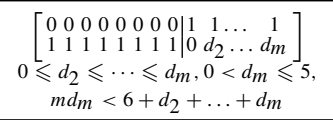 & {$\left[\begin{array}{c}m \\
6+\sum d_{k}\end{array}\right.$} & $m+5$ \\
\hline 11 & $\begin{array}{c}\frac{\mathbb{K}\left[T_{1}, \ldots, T_{7}, S_{1}, \ldots, S_{m}\right]}{\left\langle T_{1} T_{2}+T_{3} T_{4}+T_{5} T_{6}+T_{7}^{2}\right\rangle} \\
1 \leqslant m \leqslant 4\end{array}$ & {$\left[\begin{array}{ccccccc|ccc}-1 & 1 & 0 & 0 & 0 & 0 & 0 & 1 & \ldots & 1 \\
1 & 1 & 1 & 1 & 1 & 1 & 1 & 0 & \ldots & 0\end{array}\right]$} & {$\left[\begin{array}{c}m \\
5\end{array}\right]$} & $m+4$ \\
\hline 12 & $\begin{array}{c}\frac{\mathbb{K}\left[T_{1}, \ldots, T_{7}, S_{1}, \ldots, S_{m}\right]}{\left\langle T_{1} T_{2}+T_{3} T_{4}+T_{5} T_{6}+T_{7}^{2}\right\rangle} \\
m \geqslant 2\end{array}$ & 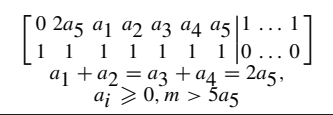 & {$\left[\begin{array}{c}m+5 a_{5} \\
5\end{array}\right]$} & $m+4$ \\
\hline 13 & $\begin{array}{c}\frac{\mathbb{K}\left[T_{1}, \ldots, T_{7}, S_{1}, \ldots, S_{m}\right]}{\left\langle T_{1} T_{2}+T_{3} T_{4}+T_{5} T_{6}+T_{7}^{2}\right\rangle} \\
m \geqslant 2\end{array}$ & 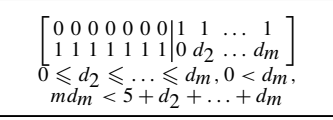 & {$\left[\begin{array}{c}m \\
5+\sum^{m} d_{k}\end{array}\right]$} & $m+4$ \\
\hline 14 & $\begin{array}{c}\mathbb{K}\left[T_{1}, \ldots, T_{10}\right] \\
\left\langle\begin{array}{c}T_{1} T_{2}+T_{3} T_{4}+T_{5} T_{6}+T_{7} T_{8}, \\
\lambda_{1} T_{3} T_{4}+\lambda_{2} T_{5} T_{6}+T_{7} T_{8}+T_{9} T_{10}\end{array}\right\rangle\end{array}$ & 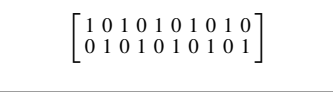 & {$\left[\begin{array}{l}3 \\
3\end{array}\right]$} & 6 \\
\hline
\end{tabular}

Moreover, each of the listed data defines a smooth Fano general arrangement variety of true complexity two and Picard number two. 
REMARK 9.2. Some of the above Fano varieties are intrinsic quadrics. Here is the overlap of Theorem 9.1 with [30, Corollary 1.2]:

(i) Cases 10 and 13 are intrinsic quadrics of Type 1,

(ii) Cases 9 and 12 are intrinsic quadrics of Type 2,

(iii) Cases 8 and 11 are intrinsic quadrics of Type 3,

(iv) Case 4.G is an intrinsic quadric of Type 4.

Let us discuss some aspects of the geometry of the Fano varieties listed in Theorem 9.1. We take a look at elementary contractions, that is, the morphisms obtained by passing to facets of the ample cone with respect to the Mori chamber decomposition, which is directly computable in terms of the data listed in Theorem 9.1; use Proposition 5.6 and Remark 5.8. Moreover, we look at small degenerations, that means degenerations with fibers all sharing the same divisor class group. In fact, degenerating the quadrinomial equations of the Cox ring into trinomial ones, reflects a degeneration of Cox rings inducing a small degeneration of the underlying Fano variety into a possibly singular variety with a torus action of complexity one.

We now explicitly go through the list of Theorem 9.1 and provide basic information in the subsequent table; more details will be made available elsewhere. Let us explain how to read the table. By $Q_{k}$, we denote the smooth projective quadric of dimension $k$ and by $Q_{k, l} \subseteq \mathbb{P}_{l}$ the projective quadric of rank $k$ in $\mathbb{P}_{l}$. We write $Y_{a ; 1^{k}, d^{l}}$ for a hypersurface of degree $a$ in the weighted projective space $\mathbb{P}\left(1^{k}, d^{l}\right)$, where we do not specify the equation, and we set

$$
\begin{gathered}
Y_{4 B}=V\left(T_{0}^{3}+T_{1} T_{2}^{2}+T_{3} T_{4}^{2}+T_{5} T_{6}^{2}\right) \subseteq \mathbb{P}_{m+6}, \\
Y_{4 F}=V\left(T_{0} T_{1}^{2}+T_{2} T_{3}^{2}+T_{4} T_{5}^{2}+T_{6} T_{7}^{2}\right) \subseteq \mathbb{P}_{m+6} .
\end{gathered}
$$

As we consider smooth Fano varieties of Picard number two, there will be at most two elementary contractions for each. If we have a birational elementary contraction, then a prime divisor gets contracted. In this case we write $X \sim Y$ and denote by $C \subseteq Y$ the center of this contraction. The other possibility is that we have a Mori fiber space. Then we write $X \rightarrow Y$ and denote by $F_{\text {gen }}$ the general fiber. If there are no special fibers, then we write just $F$ for the fiber. Moreover, when we say that a variety is Gorenstein, terminal, and so on, then we mean that it is singular but has at most Gorenstein, terminal, and so on, singularities. We computed small degenerations for every case in the lowest dimensions. The resulting varieties are always normal and Fano with a torus action of complexity one. The properties of being Gorenstein, terminal, and so on, have been checked 
using $[13,43]$. If we say two, three, and so on, degenerations, then this means that we found small degenerations into two, three, and so on, nonisomorphic Fano $\mathbb{T}$-varieties of complexity one.

REMARK 9.3. The following table lists the elementary contractions and small degenerations obtained via degenerating the Cox ring for the Fano varieties of Theorem 9.1.

\begin{tabular}{|c|c|c|c|c|}
\hline No. & $\operatorname{dim}(X)$ & Contraction 1 & Contraction 2 & Small Degenerations \\
\hline 1 & 6 & $\begin{array}{l}X \sim Q_{6} \\
C=Q_{4}\end{array}$ & $\begin{array}{c}X \rightarrow \mathbb{P}_{1} \\
F_{\text {gen }}=Q_{5}\end{array}$ & $\begin{array}{l}\text { two Gorenstein, terminal } \\
\text { locally factorial }\end{array}$ \\
\hline 2 & 6 & $\begin{array}{l}X \sim Q_{6} \\
C=\mathbb{P}_{2}\end{array}$ & $\begin{aligned} X & \rightarrow \mathbb{P}_{4} \\
F & =\mathbb{P}_{2}\end{aligned}$ & $\begin{array}{l}\text { two Gorenstein, terminal } \\
\text { locally factorial }\end{array}$ \\
\hline 3 & 5 & $\begin{array}{l}X \sim Q_{5} \\
C=Q_{3}\end{array}$ & $\begin{array}{c}X \rightarrow \mathbb{P}_{1} \\
F_{\text {gen }}=Q_{4}\end{array}$ & $\begin{array}{l}\text { three Gorenstein, terminal } \\
\text { locally factorial }\end{array}$ \\
\hline $4 \mathrm{~A}$ & $m+5$ & $\begin{array}{c}X \sim Y_{3 ; 1^{4}, 2^{m+3}} \\
C=\mathbb{P}_{m+2}\end{array}$ & $\begin{array}{l}X \rightarrow \mathbb{P}_{3} \\
F=\mathbb{P}_{m+2}\end{array}$ & $\begin{array}{l}\operatorname{dim}(X) \leqslant 6: \text { two Gorenstein, } \\
\text { terminal, locally factorial }\end{array}$ \\
\hline $4 \mathrm{~B}$ & $m+5$ & $\begin{array}{c}X \sim Y_{4 \mathrm{~B}} \\
C=\mathbb{P}_{m+2}\end{array}$ & $\begin{array}{l}X \rightarrow \mathbb{P}_{3} \\
F=\mathbb{P}_{m+2}\end{array}$ & $\begin{array}{l}\operatorname{dim}(X) \leqslant 6: \text { two Gorenstein, } \\
\log \text { terminal, locally factorial }\end{array}$ \\
\hline $4 \mathrm{C}$ & $m+5$ & - & $\begin{array}{c}X \rightarrow \mathbb{P}_{3} \\
F=\mathbb{P}_{m+2}\end{array}$ & $\begin{array}{l}\operatorname{dim}(X) \leqslant 6: \text { two Gorenstein, } \\
\text { terminal, locally factorial }\end{array}$ \\
\hline $4 \mathrm{D}$ & $m+5$ & $\begin{array}{c}\text { if } d_{1}=1 \text { or } m=0: \\
X \sim Q_{7, m+6} \\
C=\mathbb{P}_{m+2}\end{array}$ & $\begin{array}{c}X \rightarrow \mathbb{P}_{3} \\
F=\mathbb{P}_{m+2}\end{array}$ & $\begin{array}{l}\operatorname{dim}(X) \leqslant 6: \text { two Gorenstein, } \\
\text { terminal, locally factorial }\end{array}$ \\
\hline $4 \mathrm{E}$ & $m+5$ & $\begin{array}{r}X \rightarrow \mathbb{P}_{m+3} \\
F_{\text {gen }}=Y_{3 ; 1^{4}}\end{array}$ & $\begin{array}{c}X \rightarrow \mathbb{P}_{3} \\
F=\mathbb{P}_{m+2}\end{array}$ & $\begin{array}{l}\operatorname{dim}(X) \leqslant 6: \text { one Gorenstein, } \\
\text { locally factorial }\end{array}$ \\
\hline $4 \mathrm{~F}$ & $m+5$ & $\begin{array}{c}\text { if } d_{1}=0 \text { or } m=0: \\
X \rightarrow \mathbb{P}_{m+3} \\
F_{\text {gen }}=\mathbb{P}_{1} \times \mathbb{P}_{1} \\
\text { if } d_{1}=-1: \\
X \sim Y_{4 F} \\
C=\mathbb{P}_{m+2}\end{array}$ & $\begin{array}{l}X \rightarrow \mathbb{P}_{3} \\
F=\mathbb{P}_{m+2}\end{array}$ & $\begin{array}{l}\operatorname{dim}(X) \leqslant 6: \text { one Gorenstein, } \\
\log \text { terminal, locally factorial }\end{array}$ \\
\hline $4 \mathrm{G}$ & $m+5$ & $\begin{array}{c}\text { if } d_{i}=0 \text { or } m=0: \\
X \rightarrow \mathbb{P}_{m+3} \\
\quad F_{\text {gen }}=\mathbb{P}_{2} \\
\text { if } d_{1}=-1 \text { and } d_{2}=0: \\
X \sim Q_{7, m+6} \\
\text { if } d_{1}=-2 \text { and } d_{2}=0: \\
X \sim Y_{3 ; 1^{4}, 2^{m+3}}\end{array}$ & $\begin{array}{l}X \rightarrow \mathbb{P}_{3} \\
F=\mathbb{P}_{m+2}\end{array}$ & $\begin{array}{l}\operatorname{dim}(X) \leqslant 6: \text { one Gorenstein, } \\
\text { terminal, locally factorial }\end{array}$ \\
\hline
\end{tabular}




\begin{tabular}{|c|c|c|c|c|}
\hline 5 & $m+5$ & $\begin{array}{l}X \rightarrow \mathbb{P}_{m+2} \\
F_{\text {gen }}=Q_{3}\end{array}$ & - & $\begin{array}{l}\operatorname{dim}(X)=6: \text { one Gorenstein, } \\
\text { terminal, locally factorial; } \\
\text { one Gorenstein, log terminal } \\
\text { locally factorial }\end{array}$ \\
\hline 6 & $m+5$ & $\begin{array}{l}X \rightarrow \mathbb{P}_{m+1} \\
F_{\text {gen }}=Q_{4}\end{array}$ & - & $\begin{array}{l}\operatorname{dim}(X)=7: \text { two Gorenstein, } \\
\text { terminal, locally factorial }\end{array}$ \\
\hline 7 & $m+5$ & $\begin{array}{c}X \rightarrow \mathbb{P}_{m} \\
F_{\text {gen }}=Q_{5}\end{array}$ & - & $\begin{array}{l}\operatorname{dim}(X)=8: \text { two Gorenstein, } \\
\text { terminal, locally factorial }\end{array}$ \\
\hline 8 & $m+5$ & $\begin{array}{c}X \sim \mathbb{P}_{m+5} \\
C=Q_{4}\end{array}$ & $\begin{array}{l}\text { if } d_{1}=-1: \\
X \sim Q_{6} \\
C=\{\mathrm{pt}\}\end{array}$ & $\begin{array}{l}\operatorname{dim}(X)=6: \text { one Gorenstein, } \\
\text { terminal, locally factorial; } \\
\text { one of Gorenstein index } 2 \text {, } \\
\text { terminal, } \mathbb{Q} \text {-factorial }\end{array}$ \\
\hline
\end{tabular}

\begin{tabular}{|c|c|c|c|c|}
\hline 9 & $m+5$ & $\begin{array}{l}X \rightarrow \mathbb{P}_{m-1} \\
F_{\text {gen }}=Q_{6}\end{array}$ & $\begin{array}{l}\text { if } a_{1}=\ldots=a_{7}=0: \\
\quad X \rightarrow Q_{6} \\
F=\mathbb{P}_{m-1}\end{array}$ & $\begin{array}{l}\operatorname{dim}(X)=7: \text { one Gorenstein, } \\
\text { terminal, locally factorial }\end{array}$ \\
\hline 10 & $m+5$ & $\begin{array}{c}X \rightarrow Q_{6} \\
F_{\text {gen }}=\mathbb{P}_{m-1}\end{array}$ & $\begin{array}{c}\text { if } 0<d_{2}=\ldots=d_{m}: \\
X \sim Y_{2 ; 1^{8}, d_{2}^{m-1}} \\
C=\mathbb{P}_{m-2}\end{array}$ & $\begin{array}{l}\operatorname{dim}(X)=7: \text { one Gorenstein, } \\
\text { terminal, locally factorial }\end{array}$ \\
\hline 11 & $m+4$ & $\begin{array}{c}X \sim \mathbb{P}_{m+4} \\
C=Q_{3}\end{array}$ & $\begin{array}{l}\text { if } m=1: \\
X \sim Q_{5} \\
C=\{\mathrm{pt}\}\end{array}$ & $\begin{array}{l}\operatorname{dim}(X)=5 \text { : two Gorenstein, } \\
\text { terminal, locally factorial; } \\
\text { one Gorenstein, terminal } \\
\mathbb{Q} \text {-factorial. } \\
\operatorname{dim}(X)=6 \text { : two Gorenstein, } \\
\text { terminal, locally factorial; } \\
\text { one of Gorenstein index } 2, \\
\text { terminal, } \mathbb{Q} \text {-factorial }\end{array}$ \\
\hline 12 & $m+4$ & $\begin{array}{l}X \rightarrow \mathbb{P}_{m-1} \\
F_{\text {gen }}=Q_{5}\end{array}$ & $\begin{array}{c}\text { if } a_{1}=\ldots=a_{5}=0: \\
X \rightarrow \mathbb{P}_{m-1} \\
F=Q_{5}\end{array}$ & $\begin{array}{l}\operatorname{dim}(X)=6: \text { two Gorenstein, } \\
\text { terminal, locally factorial }\end{array}$ \\
\hline 13 & $m+4$ & $\begin{array}{c}X \rightarrow Q_{5} \\
F_{\text {gen }}=\mathbb{P}_{m-1}\end{array}$ & $\begin{array}{l}\text { if } 0<d_{2}=\ldots=d_{m}: \\
X \sim Y_{2 ; 1^{7}, d_{2}^{m-1}} \\
\quad C=\mathbb{P}_{m-2}\end{array}$ & $\begin{array}{l}\operatorname{dim}(X)=6: \text { two Gorenstein, } \\
\text { terminal, locally factorial }\end{array}$ \\
\hline 14 & 6 & $\begin{array}{c}X \rightarrow \mathbb{P}_{4} \\
F_{\text {gen }}=\mathbb{P}_{2}\end{array}$ & $\begin{array}{c}X \rightarrow \mathbb{P}_{4} \\
F_{\text {gen }}=\mathbb{P}_{2}\end{array}$ & $\begin{array}{l}\text { one Gorenstein, terminal } \\
\text { locally factorial }\end{array}$ \\
\hline
\end{tabular}

REMARK 9.4. All varieties of Theorem 9.1 can be constructed out of a finite set of starting varieties listed in Theorem 1.2 via iterated duplication of free weights as introduced in [31, Construction 5.1]. In this procedure, one takes a Cox ring generator $S_{k}$ of $X$ not occurring in the defining relations and constructs a new 
Cox ring by adding a further free generator $S_{k}^{\prime}$ of the same degree as $S_{k}$. The resulting variety $X^{\prime}$ is of one dimension higher. In terms of birational geometry, the duplication of a free weight means taking an elementary contraction $\widetilde{X}_{1} \rightarrow X$ with fiber $\mathbb{P}_{1}$, passing via a series of small quasimodifications to $\widetilde{X}_{t}$ and then performing a contraction of a prime divisor $\widetilde{X}_{t} \rightarrow X^{\prime}$; see [31, Proposition 5.3]. It follows from [31, Proposition 5.4, Theorem 5.5] that every smooth Fano variety of true complexity one and Picard number two is of dimension 4 to 7 or arises via iterated duplications of free weights from a finite set of smooth projective varieties of true complexity one and Picard number two of dimension 4 to 7 . For the Fano general arrangement varieties of true complexity two listed in Theorem 9.1, one directly establishes the analogous statement with a finite set of starting varieties of dimensions 5 to 8 . It would be interesting to see if the smooth Fano general arrangement varieties of Picard number two but higher complexity behave similarly.

THEOREM 9.5. Every smooth projective truly almost Fano general arrangement variety of true complexity two and Picard number two is isomorphic to precisely one of the following varieties $X$, specified by their Cox ring $\mathcal{R}(X)$, the matrix $\left[w_{1}, \ldots, w_{r}\right]$ of generator degrees and an ample class $u \in \mathrm{Cl}(X)=\mathbb{Z}^{2}$.

\begin{tabular}{|c|c|c|c|}
\hline $\mathcal{R}(X)$ & {$\left[w_{1}, \ldots, w_{r}\right]$} & $u$ & $\operatorname{dim}(X)$ \\
\hline 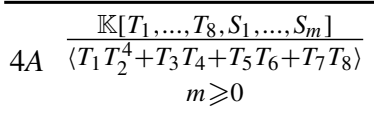 & {$\left[\begin{array}{llllllllllll}0 & 1 & 1 & 1 & 1 & 3 & 1 & 3 & 3 \\
1 & 0 & 1 & 0 & 0 & 0 & 0 & 0 & 1 & \ldots & 1\end{array}\right]$} & {$\left[\begin{array}{l}4 \\
1\end{array}\right]$} & $m+5$ \\
\hline 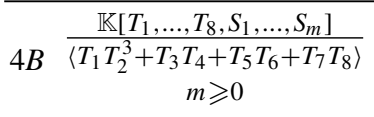 & {$\left[\begin{array}{lllllllllllll}0 & 1 & 2 & 1 & 2 & 2 & 1 & 1 & 2 & \ldots & \\
1 & 0 & 1 & 0 & 1 & 0 & 1 & 0 & 1 & 1 & \ldots & 1\end{array}\right]$} & {$\left[\begin{array}{l}3 \\
1\end{array}\right]$} & $m+5$ \\
\hline $\begin{array}{c}4 C \frac{\mathbb{K}\left[T_{1}, \ldots, T_{8}, S_{1}, \ldots, S_{m}\right]}{\left\langle T_{1} T_{2}^{3}+T_{3} T_{4}^{2}+T_{5} T_{6}+T_{7} T_{8}\right\rangle} \\
m \geqslant 0\end{array}$ & {$\left[\begin{array}{llllllll|lll}0 & 1 & 1 & 1 & 2 & 2 & 1 & 2 & \ldots & 2 \\
1 & 0 & 1 & 0 & 1 & 0 & 1 & 0 & 1 & \ldots & 1\end{array}\right]$} & {$\left[\begin{array}{l}3 \\
1\end{array}\right]$} & $m+5$ \\
\hline $\begin{array}{c}\underline{4 D} \frac{\mathbb{K}\left[T_{1}, \ldots, T_{8}, S_{1}, \ldots, S_{m}\right]}{\left\langle T_{1} T_{2}^{4}+T_{3} T_{4}^{2}+T_{5} T_{6}^{2}+T_{7} T_{8}^{2}\right\rangle} \\
m \geqslant 0\end{array}$ & 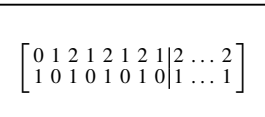 & {$\left[\begin{array}{l}3 \\
1\end{array}\right]$} & $m+5$ \\
\hline 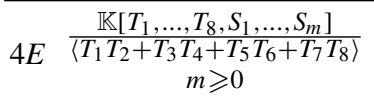 & {$\left[\begin{array}{lllllllllll}0 & 1 & 0 & 1 & 0 & 1 & 1 & 1 & 1 & & 1 \\
1 & 0 & 1 & 0 & 1 & 0 & 1 & 0 & \ldots & \ldots & 1\end{array}\right]$} & {$\left[\begin{array}{l}2 \\
1\end{array}\right]$} & $m+5$ \\
\hline 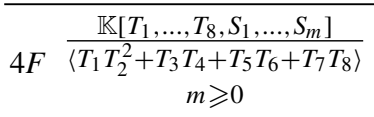 & 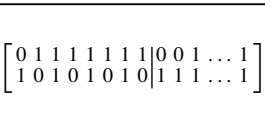 & {$\left[\begin{array}{l}2 \\
1\end{array}\right]$} & $m+5$ \\
\hline 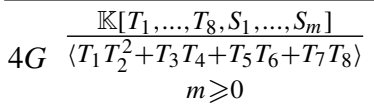 & {$\left[\begin{array}{cccccccc|cccc}0 & 1 & 1 & 1 & 1 & 1 & 1 & 1 & -1 & 1 & \ldots & 1 \\
1 & 0 & 1 & 0 & 0 & 0 & 1 & 0 & 1 & 1 & \ldots & 1\end{array}\right]$} & {$\left[\begin{array}{l}2 \\
1\end{array}\right]$} & $m+5$ \\
\hline
\end{tabular}




\begin{tabular}{|c|c|c|c|c|}
\hline $4 H$ & $\begin{array}{c}\frac{\mathbb{K}\left[T_{1}, \ldots, T_{8}, S_{1}, \ldots, S_{m}\right]}{\left\langle T_{1} T_{2}^{2}+T_{3} T_{4}^{2}+T_{5} T_{6}^{2}+T_{7} T_{8}\right\rangle} \\
m \geqslant 0\end{array}$ & {$\left[\begin{array}{llllllll|lll}0 & 1 & 0 & 1 & 0 & 1 & 1 & 1 & 1 & \ldots & 1 \\
1 & 0 & 1 & 0 & 1 & 0 & 1 & 0 & 1 & \ldots & 1\end{array}\right]$} & {$\left[\begin{array}{l}2 \\
1\end{array}\right]$} & $m+5$ \\
\hline $4 I$ & $\begin{array}{c}\frac{\mathbb{K}\left[T_{1}, \ldots, T_{8}, S_{1}, \ldots, S_{m}\right]}{\left\langle T_{1} T_{2}^{3}+T_{3} T_{4}^{2}+T_{5} T_{6}^{2}+T_{7} T_{8}^{2}\right\rangle} \\
m \geqslant 0\end{array}$ & {$\left[\begin{array}{llllllll|llll}0 & 1 & 1 & 1 & 1 & 1 & 1 & 1 & 0 & 1 & \ldots & 1 \\
1 & 0 & 1 & 0 & 1 & 0 & 1 & 0 & 1 & 1 & \ldots & 1\end{array}\right]$} & {$\left[\begin{array}{l}2 \\
1\end{array}\right]$} & $m+5$ \\
\hline $4 J$ & $\begin{array}{c}\frac{\mathbb{K}\left[T_{1}, \ldots, T_{8}, S_{1}, \ldots, S_{m}\right]}{\left\langle T_{1} T_{2}^{2}+T_{3} T_{4}^{2}+T_{5} T_{6}+T_{7} T_{8}\right\rangle} \\
m \geqslant 0\end{array}$ & {$\left[\begin{array}{llllllll|llll}0 & 1 & 0 & 1 & 1 & 1 & 1 & 1 & 0 & 1 & \ldots & 1 \\
1 & 0 & 1 & 0 & 1 & 0 & 1 & 0 & 1 & 1 & \ldots & 1\end{array}\right]$} & {$\left[\begin{array}{l}2 \\
1\end{array}\right]$} & $m+5$ \\
\hline $4 K$ & $\begin{array}{c}\frac{\mathbb{K}\left[T_{1}, \ldots, T_{8}, S_{1}, \ldots, S_{m}\right]}{\left\langle T_{1} T_{2}^{4}+T_{3} T_{4}^{3}+T_{5} T_{6}^{3}+T_{7} T_{8}^{3}\right\rangle} \\
m \geqslant 0\end{array}$ & {$\left[\begin{array}{llllllll|lll}0 & 1 & 1 & 1 & 1 & 1 & 1 & 1 & 1 & \ldots & 1 \\
1 & 0 & 1 & 0 & 1 & 0 & 1 & 0 & 1 & \ldots & 1\end{array}\right]$} & {$\left[\begin{array}{l}2 \\
1\end{array}\right]$} & $m+5$ \\
\hline $4 L$ & $\begin{array}{c}\frac{\mathbb{K}\left[T_{1}, \ldots, T_{8}, S_{1}, \ldots, S_{m}\right]}{\left\langle T_{1} T_{2}^{3}+T_{3} T_{4}^{2}+T_{5} T_{6}^{2}+T_{7} T_{8}^{2}\right\rangle} \\
m \geqslant 0\end{array}$ & {$\left[\begin{array}{llllllll|lll}0 & 1 & 0 & 1 & 1 & 1 & 1 & 1 & 1 & \ldots & 1 \\
1 & 0 & 1 & 0 & 1 & 0 & 1 & 0 & 1 & \ldots & 1\end{array}\right]$} & {$\left[\begin{array}{l}2 \\
1\end{array}\right]$} & $m+5$ \\
\hline $4 M$ & $\begin{array}{c}\frac{\mathbb{K}\left[T_{1}, \ldots, T_{8}, S_{1}, \ldots, S_{m}\right]}{\left\langle T_{1} T_{2}+T_{3} T_{4}+T_{5} T_{6}+T_{7} T_{8}\right\rangle} \\
m \geqslant 0\end{array}$ & 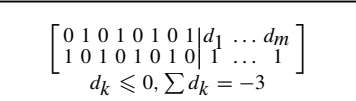 & {$\left[\begin{array}{l}1 \\
1\end{array}\right]$} & $m+5$ \\
\hline $4 N$ & $\begin{array}{c}\frac{\mathbb{K}\left[T_{1}, \ldots, T_{8}, S_{1}, \ldots, S_{m}\right]}{\left\langle T_{1} T_{2}^{2}+T_{3} T_{4}^{2}+T_{5} T_{6}^{2}+T_{7} T_{8}^{2}\right\rangle} \\
m \geqslant 0\end{array}$ & {$\left[\begin{array}{cccccccc|ccc}0 & 1 & 0 & 1 & 0 & 1 & 0 & 1 & d_{1} & \ldots & d_{m} \\
1 & 0 & 1 & 0 & 1 & 0 & 1 & 0 & 1 & \ldots & 1\end{array}\right]$} & {$\left[\begin{array}{l}1 \\
1\end{array}\right]$} & $m+5$ \\
\hline $4 O$ & $\begin{array}{c}\frac{\mathbb{K}\left[T_{1}, \ldots, T_{8}, S_{1}, \ldots, S_{m}\right]}{\left\langle T_{1} T_{2}^{3}+T_{3} T_{4}^{3}+T_{5} T_{6}^{3}+T_{7} T_{8}^{3}\right\rangle} \\
m \geqslant 0\end{array}$ & {$\left[\begin{array}{llllllll|cccc}0 & 1 & 0 & 1 & 0 & 1 & 0 & 1 & -1 & 0 & \ldots & 0 \\
1 & 0 & 1 & 0 & 1 & 0 & 1 & 0 & 1 & 1 & \ldots & 1\end{array}\right]$} & {$\left[\begin{array}{l}1 \\
1\end{array}\right]$} & $m+5$ \\
\hline $4 P$ & $\begin{array}{c}\frac{\mathbb{K}\left[T_{1}, \ldots, T_{8}, S_{1}, \ldots, S_{m}\right]}{\left\langle T_{1} T_{2}^{4}+T_{3} T_{4}^{4}+T_{5} T_{6}^{4}+T_{7} T_{8}^{4}\right\rangle} \\
m \geqslant 0\end{array}$ & {$\left[\begin{array}{llllllll|lll}0 & 1 & 0 & 1 & 0 & 1 & 0 & 1 & 0 & \ldots & 0 \\
1 & 0 & 1 & 0 & 1 & 0 & 1 & 0 & 1 & \ldots & 1\end{array}\right]$} & {$\left[\begin{array}{l}1 \\
1\end{array}\right]$} & $m+5$ \\
\hline 5 & $\begin{array}{c}\frac{\mathbb{K}\left[T_{1}, \ldots, T_{8}, S_{1}, \ldots, S_{m}\right]}{\left\langle T_{1} T_{2}+T_{3}^{2} T_{4}+T_{5}^{2} T_{6}+T_{7}^{2} T_{8}\right\rangle} \\
m \geqslant 0\end{array}$ & 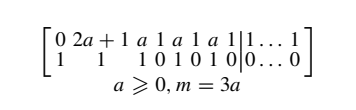 & {$\left[\begin{array}{c}2 a+2 \\
1\end{array}\right]$} & $m+5$ \\
\hline 6 & $\begin{array}{c}\frac{\mathbb{K}\left[T_{1}, \ldots, T_{8}, S_{1}, \ldots, S_{m}\right]}{\left\langle T_{1} T_{2}+T_{3} T_{4}+T_{5}^{2} T_{6}+T_{7}^{2} T_{8}\right\rangle} \\
m \geqslant 0\end{array}$ & 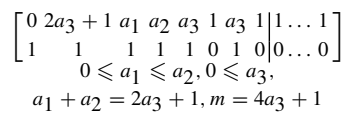 & {$\left[\begin{array}{c}2 a_{3}+2 \\
1\end{array}\right]$} & $m+5$ \\
\hline 7 & $\begin{array}{c}\frac{\mathbb{K}\left[T_{1}, \ldots, T_{8}, S_{1}, \ldots, S_{m}\right]}{\left\langle T_{1} T_{2}+T_{3} T_{4}+T_{5} T_{6}+T_{7}^{2} T_{8}\right\rangle} \\
m \geqslant 1\end{array}$ & 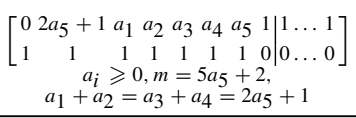 & {$\left[\begin{array}{c}2 a_{5}+2 \\
1\end{array}\right]$} & $m+5$ \\
\hline 8 & $\begin{array}{c}\frac{\mathbb{K}\left[T_{1}, \ldots, T_{8}, S_{1}, \ldots, S_{m}\right]}{\left\langle T_{1} T_{2}+T_{3} T_{4}+T_{5} T_{6}+T_{7} T_{8}\right\rangle} \\
m=6\end{array}$ & 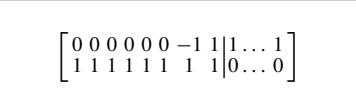 & {$\left[\begin{array}{l}1 \\
2\end{array}\right]$} & $m+5$ \\
\hline 9 & $\begin{array}{c}\frac{\mathbb{K}\left[T_{1}, \ldots, T_{8}, S_{1}, \ldots, S_{m}\right]}{\left\langle T_{1} T_{2}+T_{3} T_{4}+T_{5} T_{6}+T_{7} T_{8}\right\rangle} \\
m \geqslant 2\end{array}$ & 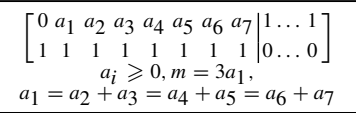 & {$\left[\begin{array}{c}a_{1}+1 \\
1\end{array}\right]$} & $m+5$ \\
\hline 10 & $\begin{array}{c}\frac{\mathbb{K}\left[T_{1}, \ldots, T_{8}, S_{1}, \ldots, S_{m}\right]}{\left\langle T_{1} T_{2}+T_{3} T_{4}+T_{5} T_{6}+T_{7} T_{8}\right\rangle} \\
m \geqslant 2\end{array}$ & 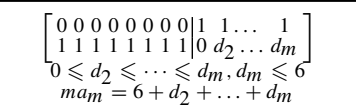 & {$\left[\begin{array}{c}1 \\
d_{m}+1\end{array}\right]$} & $m+5$ \\
\hline
\end{tabular}




\begin{tabular}{|c|c|c|c|c|}
\hline 11 & $\begin{array}{c}\frac{\mathbb{K}\left[T_{1}, \ldots, T_{7}, S_{1}, \ldots, S_{m}\right]}{\left\langle T_{1} T_{2}+T_{3} T_{4}+T_{5} T_{6}+T_{7}^{2}\right\rangle} \\
m=5\end{array}$ & {$\left[\begin{array}{ccccccc|ccc}1 & 1 & 1 & 1 & 1 & 1 & 1 & 0 & \ldots & 0 \\
-1 & 1 & 0 & 0 & 0 & 0 & 0 & 1 & \ldots & 1\end{array}\right]$} & {$\left[\begin{array}{l}1 \\
2\end{array}\right]$} & $m+4$ \\
\hline 12 & $\begin{array}{c}\frac{\mathbb{K}\left[T_{1}, \ldots, T_{7}, S_{1}, \ldots, S_{m}\right]}{\left\langle T_{1} T_{2}+T_{3} T_{4}+T_{5} T_{6}+T_{7}^{2}\right\rangle} \\
m \geqslant 2\end{array}$ & 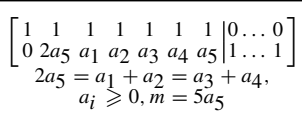 & {$\left[\begin{array}{c}2 a_{5}+1 \\
1\end{array}\right.$} & $m+4$ \\
\hline 13 & $\begin{array}{c}\frac{\mathbb{K}\left[T_{1}, \ldots, T_{7}, S_{1}, \ldots, S_{m}\right]}{\left\langle T_{1} T_{2}+T_{3} T_{4}+T_{5} T_{6}+T_{7}^{2}\right\rangle} \\
m \geqslant 2\end{array}$ & 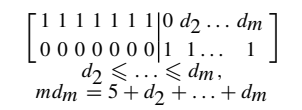 & {$\left[\begin{array}{c}1 \\
d_{m}+1\end{array}\right]$} & $m+4$ \\
\hline
\end{tabular}

Moreover, each of the listed data defines a smooth truly almost Fano general arrangement variety of true complexity two and Picard number two.

\section{Acknowledgements}

We are indebted to the referee for his very careful reading of the draft and a long list of very helpful suggestions for improvements.

\section{Conflict of Interest: None}

\section{References}

[1] K. Altmann and J. Hausen, 'Polyhedral divisors and algebraic torus actions', Math. Ann. 334(3) (2006), 557-607.

[2] K. Altmann, J. Hausen and H. Süss, 'Gluing affine torus actions via divisorial fans', Transform. Groups 13(2) (2008), 215-242.

[3] K. Altmann and G. Hein, 'A fansy divisor on $\bar{M}_{0, n}$ ', J. Pure Appl. Algebra 212(4) (2008), 840-850.

[4] I. Arzhantsev, 'On the factoriality of Cox rings', Mat. Zametki 85(5) (2009), 643-651.

[5] I. Arzhantsev, U. Derenthal, J. Hausen and A. Laface, Cox Rings, Cambridge Studies in Advanced Mathematics 144 (Cambridge University Press, Cambridge, 2015).

[6] M. Audin, The Topology of Torus Actions on Symplectic Manifolds, Progress in Mathematics, 93 (Birkhäuser, Basel, 1991), Translated from the French by the author.

[7] H. Bäker, J. Hausen and S. Keicher, 'On Chow quotients of torus actions', Michigan Math. J. 64(3) (2015), 451-473.

[8] V. V. Batyrev, 'Toric Fano threefolds', Izv. Akad. Nauk SSSR Ser. Mat. 45(4) (1981), 704-717. 927.

[9] V. V. Batyrev, 'On the classification of toric Fano 4-folds', J. Math. Sci. (New York) 94(1) (1999), 1021-1050.

[10] V. V. Batyrev, 'Toric degenerations of Fano varieties and constructing mirror manifolds', in The Fano Conference (Univ. Torino, Turin, 2004), 109-122.

[11] B. Bechtold, 'Factorially graded rings and Cox rings', J. Algebra 369 (2012), 351-359.

[12] B. Bechtold, 'Valuative and geometric characterizations of Cox sheaves', J. Commut. Algebra 10(1) (2018), 1-43. 
[13] B. Bechtold, J. Hausen, E. Huggenberger and M. Nicolussi, 'On terminal Fano 3-folds with 2-torus action', Int. Math. Res. Not. IMRN 5 (2016), 1563-1602.

[14] F. Berchtold and J. Hausen, 'Homogeneous coordinates for algebraic varieties', J. Algebra 266(2) (2003), 636-670.

[15] F. Berchtold and J. Hausen, 'Cox rings and combinatorics', Trans. Amer. Math. Soc. 359(3) (2007), 1205-1252.

[16] C. Birkar, 'Singularities of linear systems and boundedness of Fano varieties', Preprint, 2016, arXiv:1609.05543.

[17] C. Birkar, P. Cascini, C. D. Hacon and J. McKernan, 'Existence of minimal models for varieties of log general type', J. Amer. Math. Soc. 23(2) (2010), 405-468.

[18] M. Borelli, 'Divisorial varieties', Pacific J. Math. 13 (1963), 375-388.

[19] D. Bourqui, 'La conjecture de Manin géométrique pour une famille de quadriques intrinsèques', Manuscripta Math. 135(1-2) (2011), 1-41.

[20] C. Casagrande, 'On the birational geometry of Fano 4-folds', Math. Ann. 355(2) (2013), $585-628$.

[21] A.-M. Castravet and J. Tevelev, ' $\bar{M}_{0, n}$ is not a Mori dream space', Duke Math. J. 164(8) (2015), 1641-1667.

[22] W.-L. Chow, 'On the geometry of algebraic homogeneous spaces', Ann. of Math. (2) 50 (1949), 32-67.

[23] J.-L. Colliot-Thélène and J.-J. Sansuc, 'Torseurs sous des groupes de type multiplicatif; applications à l'étude des points rationnels de certaines variétés algébriques', C. R. Acad. Sci. Paris Sér. A-B 282(18) (1976), A1113-A1116. Aii.

[24] D. A. Cox, 'The homogeneous coordinate ring of a toric variety', J. Algebraic Geom. 4(1) (1995), 17-50.

[25] D. A. Cox, J. B. Little and H. K. Schenck, Toric Varieties, Graduate Studies in Mathematics, 124 (American Mathematical Society, Providence, RI, 2011).

[26] A. Craw and D. Maclagan, 'Fiber fans and toric quotients', Discrete Comput. Geom. 37(2) (2007), 251-266.

[27] V. I. Danilov, 'The geometry of toric varieties', Uspekhi Mat. Nauk 33(2(200)) (1978), 85-134. 247.

[28] M. Demazure, 'Sous-groupes algébriques de rang maximum du groupe de Cremona', Ann. Sci. Éc. Norm. Supér. (4) 3 (1970), 507-588.

[29] E. J. Elizondo, K. Kurano and K.-I. Watanabe, 'The total coordinate ring of a normal projective variety', J. Algebra 276(2) (2004), 625-637.

[30] A. Fahrner and J. Hausen, 'On intrinsic quadrics, Canad. J. Math. to appear', Preprint, 2017, arXiv:1712.09822, doi:10.4153/CJM-2018-037-5.

[31] A. Fahrner, J. Hausen and M. Nicolussi, 'Smooth projective varieties with a torus action of complexity 1 and Picard number 2', Ann. Sc. Norm. Super. Pisa Cl. Sci. (5) 18(2) (2018), $611-651$.

[32] K.-H. Fieseler and L. Kaup, 'Fixed points, exceptional orbits, and homology of affine $\mathbf{C}^{*}$ surfaces', Compos. Math. 78(1) (1991), 79-115.

[33] K.-H. Fieseler and L. Kaup, 'On the geometry of affine algebraic $\mathbf{C}^{*}$-surfaces', in Problems in the Theory of Surfaces and their Classification (Cortona, 1988), Sympos. Math., XXXII (Academic Press, London, 1991), 111-140.

[34] H. Flenner and M. Zaidenberg, 'Normal affine surfaces with $\mathbb{C}^{*}$-actions', Osaka J. Math. 40(4) (2003), 981-1009.

[35] W. Fulton, Introduction to Toric Varieties, Annals of Mathematics Studies 131 (Princeton University Press, Princeton, NJ, 1993), The William H. Roever Lectures in Geometry. 
[36] J. L. González and K. Karu, 'Some non-finitely generated Cox rings', Compos. Math. 152(5) (2016), 984-996.

[37] B. Hassett and Y. Tschinkel, 'Universal torsors and Cox rings', in Arithmetic of Higherdimensional Algebraic Varieties (Palo Alto, CA, 2002), Progress in Mathematics, 226 (Birkhäuser Boston, Boston, MA, 2004), 149-173.

[38] J. Hausen, 'Equivariant embeddings into smooth toric varieties', Canad. J. Math. 54(3) (2002), 554-570.

[39] J. Hausen, 'Producing good quotients by embedding into toric varieties', in Geometry of Toric Varieties, Sémin. Congr., 6 (Soc. Math. France, Paris, 2002), 193-212.

[40] J. Hausen, 'Cox rings and combinatorics. II', Mosc. Math. J. 8(4) (2008), 711-757. 847.

[41] J. Hausen and E. Herppich, 'Factorially graded rings of complexity one', in Torsors, étale Homotopy and Applications to Rational Points, London Mathematical Society Lecture Note Series, 405 (Cambridge University Press, Cambridge, 2013), 414-428.

[42] J. Hausen, E. Herppich and Hendrik Süss, 'Multigraded factorial rings and Fano varieties with torus action', Doc. Math. 16 (2011), 71-109.

[43] J. Hausen and S. Keicher, 'A software package for Mori dream spaces', LMS J. Comput. Math. 18(1) (2015), 647-659.

[44] J. Hausen, S. Keicher and A. Laface, 'Computing Cox rings', Math. Comp. 85(297) (2016), 467-502.

[45] J. Hausen, S. Keicher and A. Laface, 'On blowing up the weighted projective plane', Math. Z. 290(3-4) (2018), 1339-1358.

[46] J. Hausen and H. Süß, 'The Cox ring of an algebraic variety with torus action', Adv. Math. 225(2) (2010), 977-1012.

[47] J. Hausen and M. Wrobel, 'Non-complete rational T-varieties of complexity one', Math. Nachr. 290(5-6) (2017), 815-826.

[48] Y. Hu and S. Keel, 'Mori dream spaces and GIT', Michigan Math. J. 48(1) (2000), 331-348. Dedicated to William Fulton on the occasion of his 60th birthday.

[49] V. A. Iskovskih, 'Fano threefolds. I', Izv. Akad. Nauk SSSR Ser. Mat. 41(3) (1977), 516-562. 717.

[50] V. A. Iskovskih, 'Fano threefolds. II', Izv. Akad. Nauk SSSR Ser. Mat. 42(3) (1978), 506-549.

[51] M. M. Kapranov, 'Chow quotients of Grassmannians. I', in I. M. Gel'fand Seminar, Adv. Soviet Math., 16 (American Mathematical Society, Providence, RI, 1993), 29-110.

[52] M. M. Kapranov, B. Sturmfels and A. V. Zelevinsky, 'Quotients of toric varieties', Math. Ann. 290(4) (1991), 643-655.

[53] G. Kempf, F. F. Knudsen, D. Mumford and B. Saint-Donat, Toroidal Embeddings. I, Lecture Notes in Mathematics, 339 (Springer, Berlin-New York, 1973).

[54] M. Kreuzer and B. Nill, 'Classification of toric Fano 5-folds', Adv. Geom. 9(1) (2009), 85-97.

[55] D. Luna, 'Slices étales', in Sur les groupes algébriques, Mémoire, 33 (Bulletin de la Société Mathématique de France, Paris, 1973), 81-105.

[56] D. Luna and T. Vust, 'Plongements d'espaces homogènes', Comment. Math. Helv. 58(2) (1983), 186-245.

[57] J. S. Milne, Étale Cohomology, Princeton Mathematical Series, 33 (Princeton University Press, Princeton, NJ, 1980).

[58] S. Mori, 'Graded factorial domains', Japan. J. Math. (N.S.) 3(2) (1977), 223-238.

[59] S. Mori and S. Mukai, 'Classification of Fano 3-folds with $B_{2} \geqslant 2$ ', Manuscripta Math. 36(2) (1981/82), 147-162. 
[60] T. Oda, Convex Bodies and Algebraic Geometry, Ergebnisse der Mathematik und ihrer Grenzgebiete (3) [Results in Mathematics and Related Areas (3)], 15 (Springer, Berlin, 1988), An introduction to the theory of toric varieties, Translated from the Japanese.

[61] P. Orlik and P. Wagreich, 'Isolated singularities of algebraic surfaces with $C^{*}$ action', Ann. of Math. (2) 93 (1971), 205-228.

[62] P. Orlik and P. Wagreich, 'Singularities of algebraic surfaces with $C^{*}$ action', Math. Ann. 193 (1971), 121-135.

[63] P. Orlik and P. Wagreich, 'Algebraic surfaces with $k^{*}$-action', Acta Math. 138(1-2) (1977), 43-81.

[64] H. C. Pinkham, 'Normal surface singularities with $C^{*}$ action', Math. Ann. 227(2) (1977), 183-193.

[65] M. Rosenlicht, 'A remark on quotient spaces', An. Acad. Brasil. Ci. 35 (1963), 487-489.

[66] P. Samuel, Lectures on Unique Factorization Domains. Notes by M. Pavman Murthy. Tata Institute of Fundamental Research Lectures on Mathematics, No. 30 (Tata Institute of Fundamental Research, Bombay, 1964).

[67] D. A. Timashev, 'Torus actions of complexity one', in Toric Topology, Contemporary Mathematics, 460 (American Mathematical Society, Providence, RI, 2008), 349-364.

[68] D. A. Timashëv, 'G-manifolds of complexity 1', Uspekhi Mat. Nauk 51(3(309)) (1996), 213-214.

[69] J. Włodarczyk, 'Embeddings in toric varieties and prevarieties', J. Algebraic Geom. 2(4) (1993), 705-726.

[70] M. Wrobel, 'Structural properties of Cox rings of $T$-varieties', Doctoral Dissertation, Universität Tübingen, 2018. https://publikationen.uni-tuebingen.de. 